


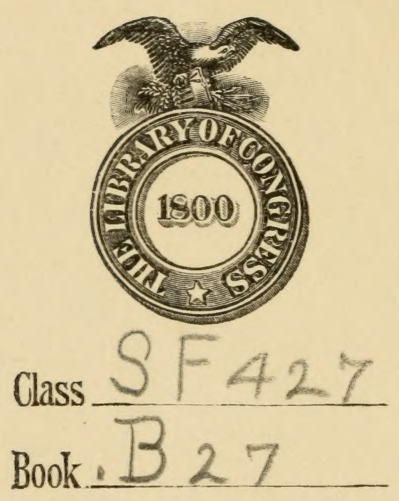

Copyight $\mathrm{N}^{0}$

COPYRIGHT DEPOSIT.

GPO 




$$
\frac{446}{2313}
$$




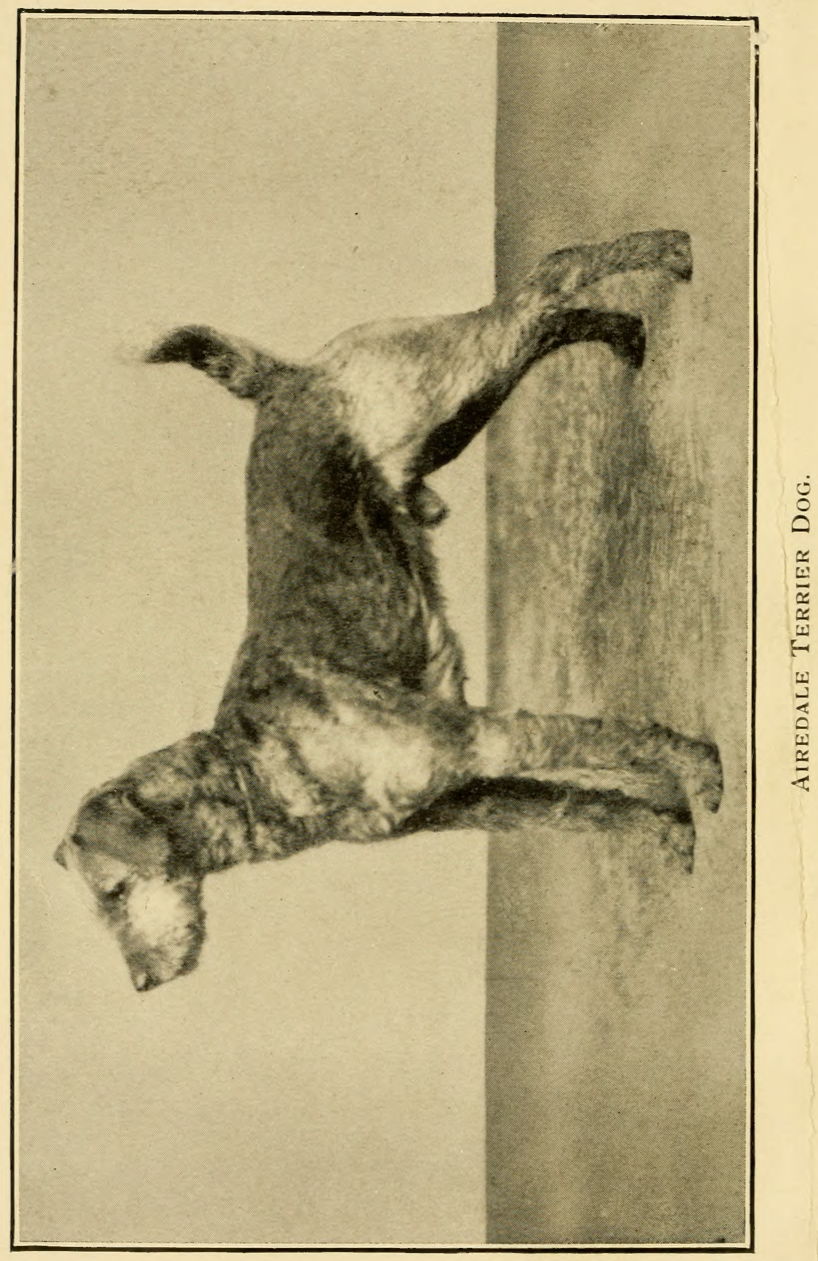




\title{
THE DOG in HEALTh,
}

\section{ACCIDENT, AND DISEASE}

\author{
BY
}

FRANK TOWNEND BARTON, M.R.C.V.S.

AUTHOR OF 'THE HORSE: AILMENTS AND ACCIDENTS'

'THE PRACTICE OF EQUINE MEDICINE'

'SPORTING DOGS'; 'NON-SPORTING DOGS'

'THE VETERINARY MANUAL,' ETC.

WITH 12 FULL-PAGE HALF-TONE ILLUSTRATIONS

$\begin{array}{lllllllllllllllllllllllll}P & H & \text { I } & \text { L } & \text { A } & \text { D } & \text { E } & \text { L } & \text { P } & \text { H } & \text { I } & \text { A }\end{array}$ H E N R Y A L T E M U S C O M P A N Y 
Copyright, 1908, by Howard E. Altemus

$$
\begin{gathered}
S F_{4} 27 \\
.327
\end{gathered}
$$

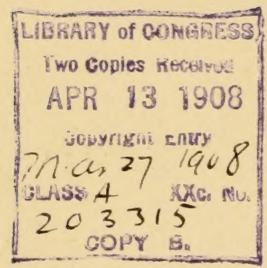

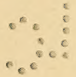




\section{PREFACE}

The Dog in Health and Disease has been prepared as an elementary treatise upon the Dog and the various diseases and accidents to which it is subject. Under the heading of the Introduction, the author has given, not only a short account of the best methods of feeding, the arrangement of kennels, exercising, grooming, washing, and other matters pertaining to the general welfare of the Dog when in health, but also advice as to treatment in times of illness. In addition to this, the points of the principal breeds of sporting, non-sporting, and other dogs have been indicated. No attempt has been made at giving anything beyond outlines throughout the book, so that the reader must not look for information that should be contained in larger and more expensive manuals. The book is intended to supply what the author believes is wanted, namely, a simple, practical treatise upon the Dog at a price within reach of almost every one.

London, 1907. 


\section{CONTENTS}

\section{INTRODUCTION}

Exercise-Grooming : or care of the Dog's Coat-Wash-

PAQE

ing-Feeding - Kennels-Administering Medicine to Dogs-Feeding Sick Dogs-Rearing PuppiesDog Shows and Preparation for Exhibition-Selecting a Dog for a Companion-Dog-breeding for Pleasure or Profit, . . . . . . .

\section{ELEMENTARY ANATOMY OF THE DOG}

Structure of the Skeleton-The Hind Limb, . . $\quad 28$

CONFORMATION AND POINTS OF THE VARIOUS BREEDS

The St. Bernard-The Newfoundland-Great Danes or Boarhounds-The Mastiff-The Deerhound-The Borzois or Russian Wolfhound-The Irish Wolfhound-The Foxhound-Otter-Hounds-The Bloodhound-The Beagle-The Harrier-The Bassett Hound-The Greyhound-The Pointer-The Setter -Retrievers-Spaniels-The Irish Water SpanielThe Water Spaniel-The Clumber Spaniel-The Field Spaniel-The Sussex Spaniel-The Cocker Spaniel-The Welsh Springer-The English Springer -The Collie-The Smooth-coated Collie-The Old English or Bob-tailed Sheep Dog-The Dalmatian vi 


\section{THE DOG}

vii

or Carriage Dog-The Whippet or Race Dog-The PAGE

Poodle-The Dachshund-The Chow-Chow-The Bull Terrier-The Manchester or Black-and-Tan Terrier-The Fox Terrier - Scottish and West Highland White Terriers - The English White Terrier-Airedale and Welsh Terriers-Dandie Dinmonts and Bedlington Terriers-Irish Terriers -Skye Terriers and Clydesdale Terriers-Bulldogs and Toy Bull-dogs - The Yorkshire Terrier -The Schipperke-The Italian Greyhound-The Pomeranian-The Pug-The Griffon BruxelloisThe Maltese Terrier-The Japanese Spaniel - The Pekinese-Toy Spaniels, . . . . . .

\section{FOREIGN DOGS}

The Samoyede - The Esquimaux - The Dingo - The Bouledoge Français - The Lhassa Terrier - The Persian Greyhound-The Hairless Dog of Mexico The Dhole-The Chesapeake Bay Dog - Thibet Spaniels-Elk Hounds, etc.-Australian Terriers, etc., . . . . . . . .

\section{DISEASES AND ACCIDENTS}

Distemper-Inflammation of the Lungs and PleurisyBronchitis-Catarrh or Coryza-Epilepsy-Chorea (St. Vitus's Dance)-Paralysis-Apoplexy-Rheumatism-Rabies (Madness)-Indigestion-Gastritis - Enteritis - Diarrhœa - Dysentery - PoisonsEczema - Parasitic Mange - Follicular Mange Lice, Fleas, and Ticks, etc.-Worms-Bareness around the Margin of the Ear-Inflammation of the Eyes-Blindness-Internal and External Canker of the Ear-Dropsy of the Flap of the Ear-Wounds, Burns, Scalds, etc. - Broken Bones or Fractures, . 


\section{LIST OF ILLUSTRATIONS}

Airedale Terrier Dog, . . . . Frontispiece AT PAGE

Rough-Coated St. Bernard, . . . . . 33

Eawn Mastiff Dog, . . . . . . . 40

Bassett Hound DoG, . . . . . . . 54

Jurly-Coated Retriever Dog, . . . . . 64

Irish Water Spaniel, . . . . . . . 72

Rough-Coated Collie, . . . . . . . 86

Shooth-Coated Collie, . . . . . . . 91

Fox Terrier DoG, . . . . . . . 109

Dandie Dinmont Terrier Dog, . . . . . 115

SKYE Terrier Dog, . . . . . . . 119

Brinded Bull-DoG, . . . . . . . . 124 


\section{INTRODUCTION}

\section{Exercise.}

REGULAR exercise is necessary for the proper maintenance of health.

Such dogs as Mastiffs, St. Bernards, Great Danes, Newfoundlands, etc., become sluggish to a degree, without a sufficiency of muscular exercise.

It is not sufficient merely to allow a dog to exercise himself by running about his master's premises. Exercise must be 'forced' upon him (or her) either by long walks or by moderate runs behind a cycle.

A most objectionable practice-if not a cruel one-is that of keeping a dog chained up from week-end to week-end.

The condition of a dog that is chained up in this way is usually unsatisfactory, and, instead of being a source of utility and pleasure to the proprietor, the animal becomes an object of pity and misery to himself and others. It is not of the slightest use to exercise a dog one day and then to keep him chained up for two or three. 
The essentials of exercise are regularity and moderation, the condition and variety of dog being duly considered. When the weather is suitable, water-loving dogs should be allowed to indulge in their aquatic habits, if a suitable pond is at hand, but it is very bad for the skin to allow a dog to paddle about in all sorts of dirty water. Puppies and young dogs should be allowed unlimited exercise. There is nothing worse than keeping them in a confined space, unless it be fastening them up with a chain. To chain a young dog up is simply fatal to the development of the limbs.

Sporting dogs require much more exercise than do non-sporting, and those who contemplate purchasing a dog for companionship will do well to avoid buying an animal of the former class. Insufficient exercise predisposes a dog to congestion of the liver, etc., and this may in course of time express itself in the form of an eczematous eruption or some other irritable condition of the skin. Aged asthmatical dogs, being incapable of undergoing much exertion, require well-regulated exercise.

\section{Grooming : or care of the Dog's Coat.}

Those who value the appearance of a dog will bestow a certain amount of attention upon the 
animal's coat, because a well-groomed dog is an additional source of pleasure to its owner. What may be termed spasmodic attention to a dog's toilet is of no use whatsoever; in fact, it is better to leave the toilet to the waywardness of nature than to carry it out by fits and starts. The coats of all smooth-haired, short-coated dogs should be gone over with a hound-glove or short, soft-haired brush, followed by a good polishing up with a chamois leather. This, with the application of a woollen coat in winter, will keep the coat fine, soft, and of good lustre. The advantages of woollen clothing in winter can hardly be overestimated; in fact, the owner and his tyke will adopt the well-known axiom of 'once used, always used.' Long rough-coated dogs require considerably more attention than do their shortcoated brethren, a fact that should be borne in mind when selecting a dog. It is necessary to have a dandy-brush and a stout steel or bone comb, the latter being used for the feather upon the limbs, tail, etc. Collies, Retrievers, roughcoated St. Bernards, and other breeds having a superabundance of long hair, must be combed regularly, otherwise the hair becomes felted; if so, the entangled masses must be cut off.

The hair of Pomeranians and Chow-chows should be brushed up or backwards in order to 
give the coat the necessary 'stand-off' appearance. To improve the glossy appearance of the coat of flat-coated Retrievers, Spaniels, or of any other breed in which brilliancy of coat is an essential point of beauty, a chamois leather should be used for about a quarter of an hour once or twice a day. Young dogs, such as Terriers whose wiry coat is a point of excellence, may with advantage have their puppy coats stripped out. This can be done through the use of the so-called 'stripping' comb, obtainable from vendors of kennel appliances.

\section{Washing.}

Although good grooming goes a long way towards cleanliness, it is not well to do away with the bath. For a dog to be healthy, its skin must be kept clean; more especially is this necessary in dogs which are kept in the house, for a dirty coat harbours fleas and other vermin, and where there are children about, these pests may cause considerable annoyance, to say nothing of the punishment frequently inflicted upon the dog. Washing destroys the natural lustre of the coat, but the lubricant reasserts its power in the course of two or three days; hence the inadvisability of washing a dog on the eve of a show. Again, hard-coated dogs should not 
be washed frequently, because washing softens the coat. The advantage of selecting a darkcoloured dog for companionship in town is at once obvious, there being no necessity for such frequent washing. For thoroughly cleansing a dog's coat, 'liquid soap' answers the purpose admirably. This, which is made by dissolving soft soap in spirit, has the further advantage of assisting in the destruction of fleas. The best soap to use under any ordinary circumstances is carbolic, but it is not advisable to use this for puppies, because absorption of the carbolic has been known to cause death. When dogs are much troubled with fleas or lice, it is a very good plan to add a quart of strong infusion of quassia to the bath. It is not necessary to employ a large amount of water; in fact, the dog can be washed better by standing it over an empty tub, thoroughly soaking the whole of the hindquarters, loins, and back, and then lathering with soap, rinsing this off, and repeating the operation on the remainder of the body, forequarters, head, ears, etc.

\section{Feeding.}

House-dog's should not be constantly fed throughout the day, but at stated times, say night and morning, or every afternoon. 
It is a good plan to feed a clog after it comes in from, but inadvisable to feed it immediately before, exercise. Certain axioms should be borne in mind as being specially applicable to the feeding of a dog:-

(a) Feed about the same hour, once or twice daily, in accordance with the condition of the animal and the amount of work it has to perform. For instance, a sporting dog during the working season requires much more food than in the summer. The same remark applies to foxhounds and harriers in and out of a hunting season.

(b) Never leave food before a dog after it has ceased feeding, because this only tends to turn it against its food.

(c) Avoid feeding entirely on flesh, but give a mixture of bread and meat, say half of each.

(d) To feed each dog separately, or, if puppies are fed together from a trough, see that the weaker ones are allowed their share. This is equally applicable to puppies in the nest.

(e) To keep a constant supply of fresh water before the dogs. 
(f) If dog biscuits are used, these should be broken up, and preferably given soaked. A considerable number of dog foods are upon the market, but the difficulty is to find a dog food that the majority of dogs will eat. Boiled rice mixed with meat, paunches boiled and cut up, hound-meal, oatmeal well boiled and mixed with meat, etc., make the best food. Greaves are still a good deal employed, and these, if obtained from a reliable source, make an excellent food, provided that they are boiled and combined with some carbonaceous material, such as boiled rice or boiled flour, etc.; but particular care must be taken not to give too much, or undesirable results will occur. If horseflesh is used, it is necessary to have it well boiled, though many dog-breeders feed their animals entirely upon raw flesh, and there can be no objection to this, if too much is not given, and the meat off a healthy carcass.

\section{Kennels.}

In the arrangement and construction of kennels a good deal will depend upon the number of dogs 
to be kept, the ground available, and the amount of money that can be spent. Where only one or two dogs are liept, inexpensive accommodation can be provided, but, if it is intended to go in for breeding dogs for profit, one must provide proper accommodation. If a small stable is handy the stalls can easily be converted into kennels, the lower end of the stall being enclosed by a framework and door, over which $\frac{1}{2}$-inch wire netting is securely fastened. Some attention must be given to the floor, because, if this is uneven, as most floors are that are paved with cobble stones, the limbs of puppies will suffer injury. Have the floor cemented, the surface drained, and a portable day-and-night bench placed at the end of the stall. Retrievers and many other long-coated dogs sleep out of doors all the year round; in fact, the only accommodation that many gamekeepers are able to provide for their dogs is a barrel. The best barrel to buy for this purpose is a petroleum one, which should be heavily tarred two or three times all over on the outside. To raise the barrel off the ground a 'tram' should be made, which consists of two stout pieces of wood, say two and a half feet long, and about five inches in thickness, placed transverse to the barrel. Upon each of these pieces place another piece cut in a semicircle to fit the lower surface of the barrel. A 
couple of screw bolts can be used to fix the 'tram ' to a railway sleeper, several of which should be laid down so as to form a square or platform for the kennel to rest upon. Kennels for single dogs can be obtained from any manufacturer.

One of the best the writer has ever seen was that exhibited by Messrs. Boulton and Paul at a recent Kennel Club show, two kennels, with yards, railings, sleeping accommodation, etc., all complete, only costing about seventeen pounds; and any one contemplating forming a stud of dogs could not do better than purchase two or more of these kennels, arranging them upon a concrete base, and in such a situation that they will face due south or south-west and have plenty of sunlight and air. Dogs for companionship, kept in the house, must be taught habits of cleanliness, and not allowed to become an annoyance by jumping up and soiling the clothes of visitors.

\section{Administering Medicine to Dogs.}

It may seem a very simple matter to give a dog a dose of medicine, either liquid or solid, but simple though it may appear, nine people out of every ten fail to do so in what may be termed a thoroughly workmanlike and business manner. Of course dogs differ as to the ease with which medicine may be administered to them, but, 
taking the average dog, there ought not to be much difficulty, if method is followed. A fluid should be given out of a small bottle, and not out of a spoon, as is commonly done. A vial holding from a half to one ounce is all that is necessary, and half an ounce, which is approximately equal to a tablespoonful, usually constitutes a dose for a medium-sized or large dog. About a fourth of the vial will be equivalent to a teaspoonful, which may be taken as the average dose for a toy dog. An assistant should hold the dog, and the owner should then grasp the left cheek with the fingers of the left hand, take the bottle in the right hand, and insert the neck between the cheeks on the right side, forming with the fingers and the neck of the bottle a sort of pouch, into which the fluid is allowed to trickle, passing down the back part of the mouth and so on down the throat. The neck of the bottle should not touch the teeth, otherwise injury may be done. Special appliances are sold for giving fluids and pills to dogs, but these are not necessary, nor of much practical utility.

\section{Feeding Sick Dogs.}

When dogs are out of health, it is necessary to resort to special means for giving the food. In many irritable conditions of the stomach the 
animal is constantly vomiting, and this, though unquestionably salutary, is exhausting, if prolonged. Under these circumstances it is advisable to give only the smallest quantities of food at frequent intervals. Boiled fish, milk and sodawater, cold arrowroot, cold arrowroot-gruel, and Brand's Essence of Beef, with 20 grains of Carbonate of Bismuth and a teaspoonful of brandy, constitutes an excellent dietary for a dog that is troubled with vomiting. Raw meat, minced up, and given in half-ounce doses, is a capital nutritive, more especially when a dog has become enfeebled by some prostrating disease, such as distemper.

\section{Rearing Puppies.}

Unless one has suitable accommodation, it is not advisable to go in for dog-breeding, because in order to rear puppies successfully they must have unlimited exercise. The writer is well enough aware that many of the best dogs that have ever been bred have been reared under conditions anything but favourable towards their development, but this is exceptional, certainly not the rule. Even toy dogs that are reared in the house do not make the progress that they would were the surroundings more suitable. Spring is the best time for the birth of puppies, so that 
they may have the advantages of the following summer for outdoor exercise, ${ }^{1}$ and if they are properly fed and carefully housed, they should almost be seen growing, so rapid should their progress be. Puppies, if allowed to lie out in the wet or in damp kennels, are liable to 'kennel lameness,' to enlargement of the joints, and, in consequence, to stunted growth. Many puppies become rickety, if they are badly fed or allowed insufficient exercise. In most kennels it is customary to rear a couple of litters of pups in one year from the same dam, in other words to have a spring and autumn litter, but the writer advocates that every bitch should have but one litter a year. If you want pups to grow, you must give them the very best of food; feed regularly three times per day, but never allow them to 'gorge' themsolves, because this predisposes them to the so-called 'pot-belly.' All pups should be weaned about the fifth week, but whilst they are suckling, say at the beginning of the third week, it is advantageous to supplement the mother's milk with some farinaceous food, such as Mellin's Food, Horlick's Malted Milk, etc. Plenty of cow's milk should be allowed as soon as

1 The author is a staunch advocate for placing puppies out at 'walk,' as this diminishes the percentage of losses through distemper, etc. 
the pups are weaned; all such substances as ' condensed' milk, etc., are practically useless, and little better than rubbish for dogs. Boiled oatmeal mixed with meat cut up in small pieces makes a capital food. Large bones to gnaw at assist teething, but don't give puppies meat.

\section{Dog Shows and Preparation for Txhibition.}

Although dog-showing cannot be regarded as a lucrative occupation, it is a neoessary business if the fancier wishes to attain the topmost rungs of fame as a breeder of high-class dogs, and he who never shows is not likely to create much of a name for himself in the 'doggie' world. It is not a wise plan to frequent the smaller shows, and certainly not a test of the merits of a dog, more especially if the classification is indifferent. The principal shows in this country are:-

The Kennel Club's Annual Show, held at the Crystal Palace in October: Cruft's Show, held at the Agricultural Hall every February; the Ladies' Kennel Association Show, usually held during the summer at the Royal Botanic Gardens; and such shows in the provinces as the Manchester, Bristol, Fylde Fox Terrier Show, Glasgow, Edinburgh, etc. What are called Specialist Club Shows are held throughout the year, the principal being the Fox Terrier Show, the Toy-Dog Show, 
the Bull-Dog Show, and the Crystal Palace and Peterborough Hound Shows. Nearly all shows are held under Kennel Club rules, and before a dog can be shown it is necessary to have its name duly entered and registered; the Secretary of the Kennel Club, Grafton Street, London, will supply all the necessary information upon application. It is not a wise plan to send a dog to a show, because some dogs will refuse to show themselves unless accompanied into the ring by their owner. Never send puppies to shows; in fact, under Kennel Club rules, you cannot send dogs less than six months old. Never show a dog unless you are thoroughly satisfied that the animal can be got into tip-top condition in time for the show. It generally takes from three to six weeks to get a dog physically fit. At least 50 per cent. of the dogs shown are not in exhibition form, and consequently are considerably handicapped, more especially when it comes to the balancing of point for point. The highest standard of exhibition condition can only be attained by plenty of grooming, regular exercise, careful feoling, and a due regard for honest principles in the Show Ring. 


\section{Selecting a Dog for a Companion.}

A distinction must be made between a dog intended purely for companionship in town, and one that has to do, in addition to this duty, a certain amount of field work in the country, or to perform the duties as a 'gun' dog for a singlehanded sportsman. Large dogs, such as Newfoundlands, St. Bernards, Mastiffs, Great Danes, and so forth, although excellent companions, cannot be recommended if the animal has to live within the domestic circle. Again, long-coated dogs like Collies, Retrievers, etc., are equally unsuitable, and certainly not healthy, if permanently kept in a dwelling-house. Dogs intended for the companionship of children must be of extremely docile temperament, because little ones are apt to take liberties that are liable to provoke peevishness in the animal. Pugs, Bull-dogs, Toy Spaniels, and many Fox Terriers make excellent companions for children, and the writer has every confidence in recommending the purchase of one of these for the home, provided that an animal of good breeding is purchased. Never buy a mongrel, no matter for what purpose required, for a mongrel is dear at any price, and nothing truer was ever said than 'that the price of a mongrel is the price of its hicle, minus the price of the rope you buy 
to hang it with.' In all probability the reader will ask what constitutes a mongrel? And also whether many of the present varieties of dogs have been derived by cross-breeding? Answering the first question, it may be said that a mongrel is the product of an alliance between two inferiorbred dogs, and that the more remote the cross from original ancestry the greater the mongrel. Returning to the second query, it must be admitted that a considerable proportion of the distinct varieties of dogs now in existence have been produced by selection and mating of distinctive breeds, but through successive generations improvements have been introduced, faults banished, and a recognised type produced, so that what might originally have been regarded as a 'cross-bred' or 'mongrel' is now an established variety. The terriers (Fox, Scottish, Airedale, Welsh, Bedlington, Irish, Bull, Manchester, etc.) all make really useful companions, and most of these can be trained to hunt a hedgerow or covert for rabbits, etc., or for 'ratting' and badger hunting, etc. As most ladies prefer the smaller varieties of dogs, and are generally on the 'lookout' for something new, various breeds from abroad have recently found favour; hence Pekinese and Japanese Spaniels and Brussels Griffons are now very popular. The Russian Wolfhound or Borzois, 
Persian Greyhounds, Elk Hounds, Samoyede, Bouledoges Français, Lhassa Terriers, Australian Terriers, and Schipperkes are all, with the exception of the last-named, friends from over the sea.

\section{Dog-breeding for Pleasure or Profit.}

There can be no doubt that dog-breeding, if it be carried on upon thoroughly systematic lines, may become a most lucrative occupation, and a source-we will not say a constant source-of pleasure to the proprietor. Every breeder of dogs has his hours of gladness and sadness, the latter becoming manifest particularly when his younger stock are visited by some destructive disease, of which distemper is the worst. Although good management goes a long way towards success, the visitation of a kennel by this malady must not be looked upon as arising through mismanagement. All things being favourable, the best, and certainly the most economical, method of establishing a kennel upon sound principles is to select, say at six months, a pup, preferably a bitch, or, what is still better, a couple of bitch-pups. It is best to apply to some breeder of repute, and, having ascertained particulars, to visit his premises, make your selection, pay the price, and bring the puppies away with you. This, with good luck, 
will constitute the nucleus of your kennel, and from it, step by step, you may hope to reach the pinnacle of fame in the particular breed which you have selected. So far, of course, you have only the feminine element in the kennel, and it now remains to make a judicious selection of a stud dog. If the alliance brings forth fruit, and luck attends your efforts, select the best only out of the litters-say a dog and a bitch pup out of each one-disposing of the other member's of the family as soon as they are weaned. Why the writer advises their immediate sale after weaning is because faults that may develop later on are not so plainly marked at this time; thus better prices can be obtained. In a sense, the plan advocated cuts both ways, as the best may, unwittingly, be disposed of in this manner. Terriers, Retrievers, and Toy-dogs are the varieties from which the best returns may be anticipated; and the cost of keep, etc., is much less than with some of the larger breeds. Pointers, Setters, St. Bernards, Great Danes, etc., are not in particular demand. Individual breeds of dogs rise and fall in a commercial sense, or, in other words, every breed of dog has its day. If fortunate enough to breod a 'flyer,' fix a reasonable catalogue price upon it at the shows, in order to tempt those on the lookout for something above the average. Never 
refuse money if a reasonable offer is made, as it frequently happens that the fortunes of life are unexpectedly turned into misfortunes.

None of the foreign breeds beyond the Spaniels, unless it be the Borzois or Russian Wolfhound, is a desirable investment for profit. Collies and Spaniels are ever popular varieties, and very good prices can be obtained for good specimens, whilst the second and third-raters out of a litter can be sold for companionship or work. The Chow-chow has always been a fashionable dog, so has the Poodle, and these command good prices. Ladies are very fond of Pomeranians, but these are not much good as companions. Yorkshire T'erriers always maintain a fair position in public estimation, and quite recently the breed has once more come to the front. A great deal might be said regarding dog-breeding as a pleasurable industry, but it is only necessary to add that second and third-rate specimens of any breed are often very difficult to dispose of, therefore the aim of the breeder should be the production of 'only the best.' 


\section{ELEMENTARY ANATOMY OF THE DOG}

THE term 'Endo-skeleton' is applied to that of the dog, horse, ox, etc., in fact, to vertebrate animals in general, in contradistinction to 'Exo-skeleton,' characteristic of the invertebrates. The firstnamed serves to support the soft structures lying outside the visceral cavities, whereas the latter encloses the soft structures. The skeleton may be regarded as the framework upon which the walls of the economy are raised, at the same time serving as a basis for the general formation of the body. T'he fore-limbs are not attached to the skeleton, their attachment being to the walls of the chest by muscle, whereas in man the top part of the limb is articulated to the skeleton. ... The hind-limbs, however, articulate through the medium of the pelvic girdle, a bone composed of three segments on each side, called the ilium, ischium, and pubis, the anterior boundary of which forms the inlet, and the posterior boundary the outlet of the pelvic cavity. The 
skull of the dog varies considerably in its shape, in accordance with the variety of the animal. For instance, in some breeds it has an inclination to be globular, whereas in others it is elongated. What is called the 'peak' or sagittal crest is very prominent in some breeds, whereas in others it is quite flat. The skull is composed of a number of bones, and these during early life can be separated from one another, but soon after birth their distinction becomes less marked, and in course of time more or less obliterated. The lower jaw is always well developed, and in almost every breed it should be about as long as the upper; if it projects beyond the latter, the dog is said to be 'undershot,' whereas, if it is too short and the upper jaw projects, the term 'overshot' is applied. With certain exceptions both these conditions are most serious faults, but in the Brussels Griffon slight protrusion of the lower jaw is regarded as a good point. Without wishing to seem presumptuous, the author would ask why such a detriment should be looked upon with favour? The same remark applies to the bull-dog, and the sooner the protruding jaw becomes a disqualifying feature, the better it will be for the welfare of the dog. It favours premature shedding of the teeth, and handicaps a dog during combat. 
The following are the vertebrae, in their order from head to tail: seven neck or cervical, thirteen dorsal, to each of which a pair of ribs is attached, the loins or lumbar vertebrae, the sacrum, and the tail bones, but in certain breeds, such as the bobtailed sheep-dog, and, very often, the Schipperke, the last-named are sometimes suppressed. The sternum or breast-bone is composed of eight segments, to which eight ribs are attached, and these ribs are spoken of as 'true,' in contradistinction to the other five which are termed 'false.' The sternum forms the floor of the chest, the arms the anterior boundary, the ribs the lateral walls, and the bodies of the dorsal vertebrae, the roof of the chest cavity, so that the chest organs are well protected by the skeleton, much better, in fact, than the organs within the abdominal cavity.

The fore-limbs correspond to the hands and arms in man, but the collar-bone is either altogether absent, or else very rudimentary. The bones composing the limb are the shoulder-blade or scapula, the arm or humerus, the fore-arm or radius, together with the ulna at the back of it, the top of the last-named forming the point of the elbow. Next are the bones of the knee, or more correctly the wrist, followed by the five metacarpal bones, the first one, that is the one 
on the inner side, being the shortest. The digits or fingers are five in number, corresponding to the human hand.

\section{The Hind-Limb.}

The femur or first thigh, articulates with the pelvic bone in a ball-and-socket joint; it is a fairly strong bone, forming, with the upper end of the tibia or second thigh bone, plus the patella or kneecap, the stifle. The femur is in a much more sheltered position than the second thigh bone, therefore less liable to injury. The patella or kneecap is a very small bone. At the side of the tibia there is a slender bone known as the fibula, which in the dog articulates with one of the bones of the hock, a joint that corresponds with the ankle in man. The remainder of the bones, from the hock downwards, are practically identical with those in the fore-limbs.

A fracture in any of the long bones of the hind-limb is much more troublesome to deal with than fractures of the fore-limb. 


\section{CONFORMATION AND POINTS OF THE VARIOUS BREEDS}

UNDER this heading the writer proposes to give a brief survey of some of the chief points and faults of the principal breeds of dogs. The popularity of a breed is not constant, and the dog that is in fashion this year may be out of fashion next. At present the medium-sized and smaller varieties of dogs seem to be most in favour, and perhaps the preference shown to these varieties is sensible. The following figures, taken from a recent catalogue of the Kennel Club's shows, are instructive:-Setter classes (exclusive of braces and teams), 45 , whereas a few years ago there were probably more than double this number; Mastiff classes, about 30, although ten or fifteen years ago these classes were amongst the best-filled in the show ; Pomeranians, on the other hand, nearly 190, proof of the extreme popularity of the breed. Foxterriers continue to be as popular as ever, but the smooth-haired variety has been somewhat displaced by its wire-haired brethren. Numerous other instances might be quoted, but the fore- 



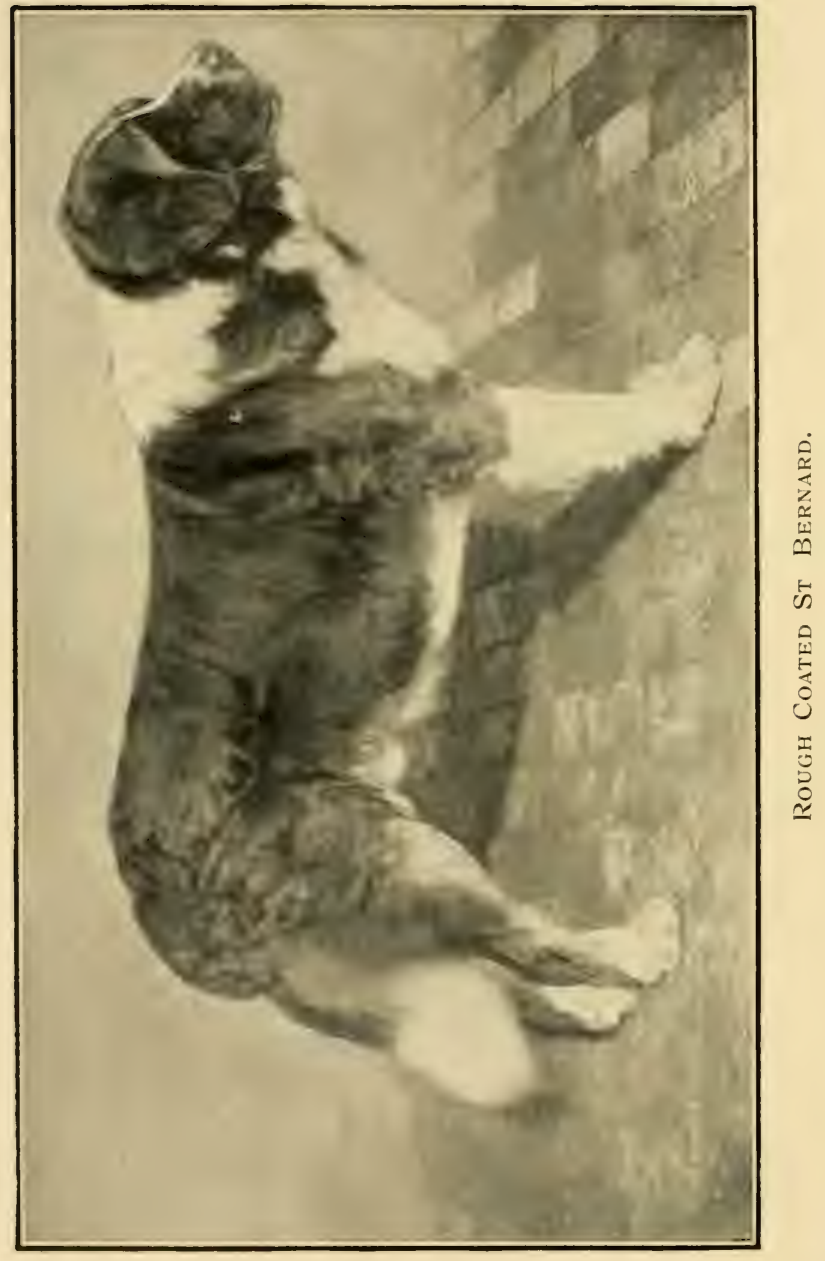




\section{POINTS OF THE VARIOUS BREEDS 33}

going will be sufficient to show that each variety has its season of sunshine.

\section{The St. Bernard.}

These monarchs of strength and beauty have always been popular in this and other countries, where their chief use is for companionship, or for the guardianship of property. It is hardly necessary to say that the breed had its origin on Mount St. Bernard, being used at the Hospice by the monks for rendering first aid to belated travellers. Many and many a life has been saved by these dogs, which truly represent the dog as a friend of man. The best specimens of St. Bernards have been bred in this country, and wonderful examples have, from time to time, appeared upon the British show-benches. Enormous prices, notably that paid by an American for the world-renowned Plinlimmon, have been obtained for first-class specimens. There are rough and smooth St. Bernards, but smooth specimens occasionally appear in a litter bred from rough-coated parents. The typical colour for a St. Bernard is orange, red, or some shade of brindle, or else white with any of the colours just mentioned, the last-named being important. There should be a white blaze at the face, white collar, white muzzle, white forelegs and feet, and, 
upon the ears, a shading of black. The coat, if short, must be flat, hard, and dense; if rough, dense and flat. A St. Bernard must not be too 'leggy;' nor must it be too short in the legs; to be either is a fault. The correct height is about thirty-two inches, as measured at the shoulder, and the average weight for a dog, in fairly good form, may be set down as 200 lbs., but a couple of stones either way is not of much importance. Plinlimmon weighed $210 \mathrm{lbs}$., and was $33 \frac{1}{2}$ inches at the shoulder.

The head ought to be massive, and the 'stop' well marked. The 'haw' ought to be plainly seen, and the eyes dark and small, deeply set in the orbits. A St. Bernard must be massive in all proportions, and the bones of the limbs big and perfectly straight. Any tendency to lightness of forearm constitutes a fault, but one of the worst faults that a St. Bernard can have, and it is most certainly a very, very common one, is the bending in an outward direction of the limbs below the hocks. A dog whose limbs bend in this way is said to have 'cow-hocks,' and the deformity is unquestionably hereditary, and every judge looks upon it with disfavour. Another fault is a fleshcoloured nose ('Dudley' nose), which constitutes a disqualifying feature. When a dog is altogether fawn in colour, and has only black shadings, or 


\section{POINTS OF THE VARIOUS BREEDS 35}

is black, black and tan, black and white, black, white and tan, or all white, it will be disqualified, if shown. Very common faults are, a curly tail, curly coat, wall eyes, flat or domed skull (likewise a self-coloured dog, without black points or shadings). It is hardly necessary to say that powerful back and loins, and gracefully-sloping quarters, constitute ideals of beauty.

\section{The Newfoundland.}

This variety of dog has never attained the popularity of the St. Bernard, and there does not appear any likelihood that he ever will. Newfoundlands are large, handsome dogs, either altogether black, or black-and-white, the latter being often spoken of as the Landseer Newfoundland, because this animal figures in a picture by Landseer. Inferior specimens of Newfoundlands are very prevalent, and the inferiority is mostly in want of size and general massiveness. The coat should be flat and dense, and of a waterresisting nature. Small dark-coloured eyes, deeply set, small closely-set ears, and large feet are typical of good breeding. The average height for a dog is 27 inches, and the weight from 120 to $150 \mathrm{lbs}$., with an average of $135 \mathrm{lbs}$. In retrievers and certain other breeds which are black in colour, raven blackness of coat con- 


\section{6}

\section{THE DOG}

stitutes an important point, but in the Newfoundland this remark does not apply, a rusty or bronze tinge, with or without a splash of white on chest or toes, not being looked upon with disfavour. In a black-and-white Newfoundland even distribution of the markings is important, and judges usually pay a good deal of attention to this. The skull should be massive, broad, and flat, and the muzzle square. The forearms, first and second thighs, must be big in the bones; in fact, massiveness throughout the whole conformation expresses the general build of a Newfoundland. The faults are: curly coat, curly tail, cowhocks, want of massiveness in head, body, or limbs, bad temper, etc. There is a Newfoundland Club, and this body allots 34 per cent. of points for the head. These dogs make excellent companions, and as a rule they are very good-tempered; make good yard dogs, but are inclined to become savage if kept too much on the chain. It is their nature to be fond of water, therefore they are easily trained to retrieve from it; in fact, the Newfoundland has attained a notoriety-in all probability a good deal overestimated, and largely of a pictorial nature-as a life-saving dog, and the Humane Society have distributed awards to Newfoundlands for meritorious conduct of this kind. Puppies are easy to rear, and thrive admirably in 
cold climates, whilst excellent specimens can be bought at prices ranging from several guineas up to fifteen or twenty, whilst more than treble the latter sum is occasionally paid for a show dog.

\section{Great Danes or Boarhounds.}

This is a short-haired, smooth-coated variety of hound, formerly used for hunting the wild boar, but now kept in this country either as a companion, or for the guardianship of property. They are powerfully-built dogs, but quite unlike the St. Bernard, Mastiff or Newfoundland, being built upon racing lines. For a long time they have been very popular dogs, more especially with women; in fact, ladies have been some of the principal exhibitors in this country. Most boarhounds have a sweet temperament, are not difficult to rear, and make excellent companions and good house-dogs; and puppies of fashionable breeding can be bought at reasonable prices. A typical boarhound should suggest to the observer strength, speed, and staying power; and, unless it possesses these three points in the highest degree, it lacks the characteristics of the breed. In colour this variety of dog varies considerably, some fanciers preferring one, others another colour. Brindle, fawn, blue, harlequin, and black are the principal colours. In its native land the dog, to be 
correct, should be white, with black markings. Harlequin boarhounds are white with black, grey, or, occasionally, fawn and brindle splashes, but none of these markings, excepting the black, and less frequently the grey, is desirable. At one time it was customary to crop these dogs, but the practice has been abolished in this country. The coat ought to be flat, short, thick and hard, and the weight from 120 to $160 \mathrm{lbs}$., according to sex, and the height at the shoulders from 30 to 36 inches. A dog, if under 30 inches, will be disqualified from competing. The limbs fore and aft must be perfectly straight, large in the bones, and, when the dog is walking or running, carried in a line with the body. Feet should be large and round, toes compact and well arched. The chest ought to be deep, and the ribs well sprung; any tendency towards flat-sidedness being faulty. Connoisseurs are very particular about quality in the region of the head, which must have great strength of muzzle, and be long in the nose, but length of head varies with the height of the dog, about 12 inches being a fair measurement from tip of nose to back of skull, which ought to be somewhat flat. The bridge of the nose, and the nostrils, must be wide. Much importance is attached to the lips and lower jaw; the former should hang quite squarely in front; 


\section{POINTS OF THE VARIOUS BREEDS 39}

and the underline of the lower jaw be in a straight line from the angle of the lip to the angle of the jaw-bone; in other words, dogs should appear to be clean-cut along the line of the lower jaw, and have the angle of the bone clearly defined. ${ }^{1}$ Small, high-set ears, carried close to the side of the head, with small, deeply-set, dark-brown eyes, and a keen expression generally, are points of beauty. A lofty carriage of the neck, slightly oblique shoulders, arched loins, long, porverful, strong first and second thighs, broad clean joints, and a high set-on, strong, tapering tail (which must never curve over the back), carried straight out, when dog is at exercise, and have a slight scimitar-like curve towards the tip, when the dog is at rest, are, individually, and collectively, qualifications for the show-ring. A broad-chested, small-sized dog, or one with greyhound-like ears, lips cut away in front, and a tail that curls over the back, is a very faulty specimen of this breed.

\section{The Mastiff.}

This grand old breed of dog used to be exceedingly popular, more especially amongst the wealthier classes, and from time to time some wonderful specimens have appeared upon the

' Judges insist upon these dogs being 'clean' on the ' underline.' 
show-bench, but within the last few years the breed has steadily declined. This is to be regretted, because the Mastiff, owing to its ancient lineage, stands pre-eminently out as a variety of dog particularly suitable for the guardianship of the mansion, and the mastiff has mostly been owned, and bred, by the wealthier classes. There are fawn and brindle varieties, but the fawns have always been in the greatest demand, their black points making them exceedingly handsome. Tiger-brindle mastiffs would appear to be more suitable for night work, and that is why gamekeepers have employed this cross for the production of the so-called 'night' dog, used for companionship, and the protection of the gamekeeper during his nocturnal wanderings. Right good 'pals' these dogs make, for their presence usually strikes terror into the heart of the poacher. The number of entries at the Kennel Club Show in 1906 was only thirty.

What has been called the 'Lymehall' strain is said to have been bred by the Leighs, of Limehall in Cheshire. The temperament of mastiffs varies, and, like other dogs, they are liable, when kept on chain, to become savage. The fawn mastiff is of two shades, corresponding to the shades of the pug. The average height for a dog is about 30 inches, and its weight from 155 to $175 \mathrm{lbs}$. 


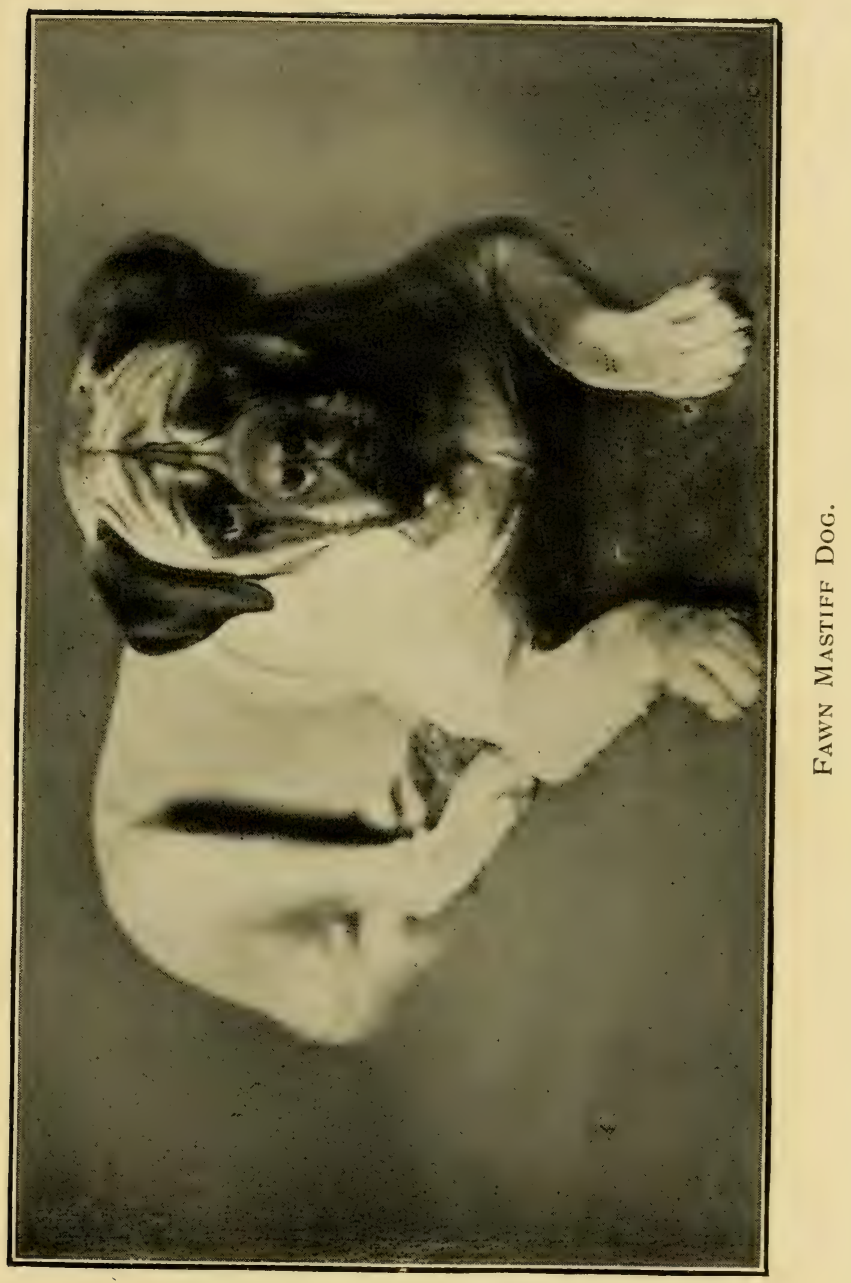





\section{POINTS OF THE VARIOUS BREEDS 41}

To be typical a mastiff must be of massive build throughout, in other words, have a heavy bony framework, and a well-developed muscular system. The eyes should be small, dark-hazel in colour, with no evidence of 'haw'; the ears small and thin; the nose broad and flat; the muzzle short and broad; the contour of the head square, the forehead being flat, and the skull broad between the ears; the skin covering the forehead loose, and slightly wrinkled; the chest wide and deep, square in its outline, and with wellsprung ribs. Straight fore-limbs, with powerful hind-quarters, well-directed hocks, and a tail carried slightly upwards under excitement, are additional points of beauty. One of the commonest faults of the mastiff is the so-called 'cow hocks,' and any tendency towards this defective conformation would necessarily handicap a dog, however good it may be in other points. The constitution of the mastiff is usually good, and puppies are not difficult to rear.

\section{The Deerhound.}

These rough-coated hounds are now chiefly used for companionship, though formerly they were used in Scotland for stag-hunting, hence the name 'deer' or 'stag' hound. Large foxhounds are often used for hunting the stag, 
and spoken of as staghounds. They are large dogs built upon racing lines, weighing from 80 to 100 lbs., and with a thick, hard, wiry coat, that gives a shaggy appearance. The average height, as measured at the shoulders, is 30 inches, but bitches are generally a trifle smaller. Brindle and steel-grey are the usual colours, but fawn, less frequently white, specimens are occasionally seen on the show-benches. In an old coloured print before the writer, a couple of deerhounds, pictured as present at the death of a stag, are white, with brindle or blackish markings upon the ears, side of the face, and the ribs and quarters. Although somewhat undersized, the dogs represented are in all respects closely allied to the type of deerhound now in existence. The deerhound's head should be long and flat on the top of the skull; the muzzle indicative of great strength; the eyes dark brown or hazel; the ears small, and carried close to the side of the head. The carriage of the tail is important; it must never curl over the back, but have a degree of the so-called 'gay' carriage when the dog is under excitement. The withers are rather high; the neck long; the shoulders deep and strong; the back, loins, and quarters long; and the breast of medium width. The general contour of the dog is like that of the greyhound, but of a distinctly 
heavier type. The fore and the hind limbs are placed as in the greyhound, but there is much more bone and substance in them, owing to the nature of the work for which the breed has been designed.

\section{The Borzois or Russian Wolfhound.}

These hounds have become very popular in England, the chief patrons being the upper classes. They are exceedingly handsome animals, and built upon the lines of the greyhound and deerhound, though of a much heavier type than the former. In Russia the Borzois is used for hunting the wolf, a brace of these dogs being slipped directly a wolf is driven out of covert. Their enormous strength enables them to hold on until the wolf is despatched by the hunter. The coat must be long and silky, and flat or wavy, but curls are not objected to. Around the neck there should be a frill; and the hair on the head, ears, and fore-limbs should be short and smooth; on the chest, body, quarters, and thighs, long; and on the tail heavily feathered, for a dog to stand up well in the prize-list. In colour, the Borzois is white, with patches of grey or orange on the body, some being heavily marked, others only feebly so. The head must be long, and finely chiselled from its back, to the nostrils; the 
length is mainly in the nose. All Borzois have a very effeminate expression. The eyes are dark, and oblique in shape: the ears are thin, set far back on the head, and covered by fine hair. The neck is long, giving the head a lofty carriage, and the shoulders are deep and sloping. Owing to the high speed at which these dogs have to work, it is necessary that the chest be of great capacity, and it is both deep and wide. Powerful loins, clean, straight fore-limbs, long, muscular first and second thighs, strong pasterns, and toes closely set together, well-arched, and thick below, are amongst the more prominent features of the breed. The tail must never be carried gaily, nor curl over the back. The constitution of the Borzois is not of the hardiest, and a considerable percentage of puppies succumb to the pneumonic complications of distemper; hence typical specimens are somerwat expensive to purchase. In this country these dogs are used only as companions. Their temperaments vary, some being extremely docile, others vicious to a degree.

\section{The Irish Wolfhound.}

This variety of dog, which in all probability has been produced by a cross from the deerhound, is said to have had its origin in Ireland, being used there for the destruction of wolves, about a 


\section{POINTS OF THE VARIOUS BREEDS 45}

couple of centuries since. The present strain of wolfhound is the product of crossing a deerhound with a boarhound, and the Irish Wolfhound Club was formed for the purpose of watching over the interest of the breed thus produced, which in most respects is closely allied to the deerhound. The following is the Club's standard:-Height, 31 inches for dogs, and 28 inches for bitches. ${ }^{1}$ Weight for dogs $120 \mathrm{lbs}$, and for bitches $90 \mathrm{lbs}$. Head, long; muzzle, long; skull, not too broad. A deep chest; a wide breast; a long, strong, muscular, well-arched neck; arched loins; and long strong quarters and thighs; large round feet, well directed; and straight, big-boned forelimbs. With reference to colour, grey, white, fawn, brindle, red, black, etc., are recognised. The Irish wolfhound has never attained any degree of popularity, and there is no particular reason why it should, there being no work suitable for it in this country. It is not so handsome a companion as the borzois.

\section{The Foxhound.}

The following description is given in the Foxhound Stud Book, and, as it is very accurate, the writer has taken the liberty of reproducing it :-

'The head should be of full size, but by no

1 The Club is anxious to increase the height to 34 inches. 
means heavy. Brow pronounced, but not high or sharp. There must be good length and breadth, sufficient to give the dog-hound a girth in front of the ears of fully 16 inches. The nose should be long (4 $\frac{1}{2}$ inches) and wide, with open nostrils. Ears set on low, and lying close to the cheek. The neck must be long and clean without the slightest throatiness; it should taper nicely from the shoulders to the head, and the upper outline should be slightly convex. The shoulders should be long and well clothed with muscle, without being heavy, especially at the points; they must be well sloped, and the true arm between the front and the elbow must be long and muscular, but free from fat and lumber. The chest should girth over 30 inches in a 24-inch hound, and the back ribs must be very deep. The back and loin must both be very muscular, running into each other without any contraction or nipping between them. The couples must be wide, even to raggedness, and there should be the very slightest arch in the loin, so as to be scarcely perceptible. The hindquarters or propellers are required to be very strong, and as endurance is of even more consequence than speed, straight stifles are preferred to those much bent, as in the greyhound. Elbows set quite straight, and neither turned in nor out, are a sine qua non; they must be well let down 


\section{POINTS OF THE VARIOUS BREEDS 47}

by means of the long true arm above mentioned. Every master of foxhounds insists on legs as straight as a post, and as strong; size of bone at the ankles and stifles being specially regarded as all-important. The feet, in all cases, should be round and catlike, with well-developed knuckles, and strong pads and nails are of the utmost importance. The colour and coat are not regarded as very important, so long as the former is a hound colour, and the latter is short, dense, hard, and glossy. Hound colours are black, tan, and white, black-and-white, and the various "pies" compounded of white and the colour of the hare and badger, or yellow or tan. In some old strains the blue mottle of the Southern hound is still preserved. The stern is gently arched, carried gaily over the back and slightly fringed with hair below. The end should taper in a point. The symmetry of the foxhound is considerable, and what is called "quality" is highly regarded by all good judges. The weight of a dog-hound from 70 to $80 \mathrm{lbs}$., of a bitch-hound from 60 to 70 lbs.'

\section{Otter-Hounds.}

The sport afforded through the use of these hounds is excellent, and it is a good deal followed in certain parts of the country. There are numerous packs of otter-hounds, and the 
followers of this form of chase aver that it is equal to any form of hunting. It is certainly a very ancient sport. It is somewhat singular that the only otter-hounds shown at the recent Kennel Club shows are those belonging to the Dumfriesshire pack. The representatives sent are usually very typical specimens. The average height is about 24 inches, and the weight from 50 to 80 lbs. The bitches generally weigh a trifle less than the dog-hounds.

A great deal of importance is attached to the quality of the coat. It must be very hard, waterresisting, and of a shaggy nature. The colours of the otter-hound vary considerably, some being sandy, others grizzle and tan, blue and white, fawn and white, black and tan, etc. The grizzle and tan is the most popular colour.

The body ought to be stoutly built, and the hound well coupled throughout. A sound set of large teeth, powerful jaws, a profusion of shaggy hair over the face, and a keen expression, are essential qualifications in the region of the head. The fore and hind limbs must be built on powerful lines, ending in large feet that are particularly hard below. The general conformation of these hounds should be that expressive of great power and endurance. 


\section{The Bloodhound.}

According to the classification adopted by the Kennel Club, the bloodhound is regarded as a sporting dog, but he is certainly not employed in this country for sport. The variety is ancient, and has been repeatedly employed for the purpose of tracking criminals, but, all things considered, the results have hardly been satisfactory, or, at any rate, not so satisfactory as to warrant the use of the dog by the Criminal Investigation Department, although demonstrations have often been given by enthusiastic breeders with a view to establishing the animal's usefulness. A remarkable feature of the bloodhound's scenting power is the ability to work out the trail or track of a stranger, whereas other breeds of dogs are only able to follow the footprints of their masters. The scent on pavements and crowded thoroughfares becomes lost, hence the drawback to their employment for criminal work. 'Bloodhounds were used by Henry vir. in the wars in France, by Queen Elizabeth against the Irish, and by the Spaniards in Mexico and Peru' (American Book of the Dog). It is a popular, but erroneous, opinion that the bloodhound is a vicious dog; in fact, it would be a difficult matter to find a dog of a more amiable disposition, and when one of these hounds is placed on the trail of a man, say on a country 
highway, it will easily find him, but not do him the slightest injury on coming up with him. It is interesting to note that, in hunting, the bloodhound relies entirely upon the sense of smell, and the greyhound entirely upon the sense of sight, wherefore the one holds his head low, and the other high. The constitution of bloodhounds is not of the most robust, and a number of puppies die through distemper. The head of a bloodhound is distinct from that of every other variety of dog; the heavily-wrinkled head, the high peak, the almost completely concealed eyes and visible 'haw', are prominent features. The dome of the skull is rather narrow, and most of the wrinkling begins on a level with the ears, which hang down, and are very long, thin, and covered with fine soft hair. The nose is broad, and the nostrils black; the eyes small, hazel in colour, and deeply set in their orbits. The flews or cheeks are long, hanging two or three inches below the level of the jaws. The facial expression of a bloodhound may not, inaptly, be described as one of wisdom. In general build, these hounds must be considered as distinctly slack, in opposition to the term 'well coupled,' so characteristic of the foxhound. The neck is long, the chest wide and deep, and the fore-limbs ought to be perfectly straight, and stoutly built. Back and loins 


\section{POINTS OF THE VARIOUS BREEDS 51}

strong; the area of the flank extensive. The stern, like that of other hounds, should be carried gaily. The colour should be black and tan, or tan only. If black and tan, the black should run along the back, top of the head, neck, and sides, but the colouring is usually blended. Lioncoloured bloodhounds are occasionally met with. The coat should be short and hard, excepting on the head and ears, where it is much softer in texture.

\section{The Beagle.}

These small hounds are chiefly used in packs for rabbit-hunting, and there are rough and smooth varieties, but the smooth hounds are in most demand, as proved by the number of entries at the Kennel Club Show. The term 'pocket beagle' is applied to the smaller variety of hound, and for several generations these little dogs have been held in great esteem. There is no doubt they have been derived from the foxhound, several characteristics of which they exhibit in a marked manner. A typical beagle may be any hound-colour, but it must have a close, hard coat, a short back, strong loins, and a gaily carried stern. In fact, the nearer these little hounds approach the build of the foxhound in a modified form, the more typical they become. 
The head should be long, and the skull well domed, yet in outline the head must be square, bearing long, low set-on ears, covered by fine short hair, and be carried like that of the foxhound. As these little hounds have to undergo a good deal of exertion, it is essential that the chest be deep, favoured by long and well-sprung ribs. The fore and hind limbs must be straight, of good bone and substance, and the hound well coupled throughout. The eyes, either of brown or of hazel, and the nostrils should be large, and the whole face have a hound-like expression. The feet should be hard and compact. Taken altogether, the beagle is a natty little dog. The most popular colour is black, white, and tan, but lemon-and-white, and blue-and-lemon mottles are frequently met with. The chief defects of these hounds are: a flat skull, short ears, eyes too close together, narrow loins, poor elbows, spreading feet, cow-hocks, lightness of bone, and a general want of hound character. Regarding the constitution of these dogs it is not necessary to say very much, because in all respects they resemble the foxhound, and, as the Kennel huntsman knows, it is chiefly during youth that ailments occur, distemper claiming a considerable number of victims. There are numerous packs of beagles throughout this country and America, but these are small, when 


\section{POINTS OF THE VARIOUS BREEDS 53}

compared with the numbers of foxhound and harrier packs.

\section{The Harrier.}

Of the Harrier, which is principally used in this country for hare-hunting, there are numerous packs. Harriers are, in reality, diminutive foxhounds, and the description of the foxhound applies to the harrier, except that the head of the latter is somewhat broader, and the coat a little coarser in texture.

\section{The Bassett Hound.}

Bassetts are short-limbed, long-bodied, smooth or rough-coated hounds, with crooked fore-limbs, and long thin ears. The breed is said to have been introduced from France, where it had existed for many centuries. Bassetts were known in France down to the seventeenth century as 'Chiens d'Artois,' but since then this name has been applied to a larger variety of hound-the Picardy hound. In many respects the Bassett hound resembles the Dachshund. Its introduction into England was chiefly due to Mr. Everett Millais, who brought a hound called 'Model' back with him from Paris. Lord Onslow at one time had a pack, and the late Mr. G. Krehl did a great deal towards the improvement of the breed. The Bassett Hound Club, formed in 1883, 


\section{THE DOG}

was composed of Lord Onslow, Messrs. Krehl, Watson, Barton, Crake, and others. ${ }^{1}$ The huntsman elected was Mr. Alleyn, the Club having decided to form a pack for hunting. Bassett hounds are sweet-tempered dogs, and even when they bring their quarry to bay they refuse to draw blood. In constitution they are somewhat delicate, a large percentage of them dying through distemper. The typical markings are tri-colour, the body being white, with one or more patches of black and tan. The side of the head and the face and ears are usually tan, with a white muzzle and ratch up the face; but there are numerous varieties of markings upon a white groundwork with black and tan. The average height is from 11 to 12 inches at the shoulder, and the weight between 40 and $45 \mathrm{lbs}$. In proportion to size the Bassett is a big-boned dog, with great strength at the shoulders and hind-quarters. The head should be long and narrow, and the occipital peak well developed. Nose broad; eyes dark brown, deeply set in the orbits, showing a good deal of 'haw.' Ears long, thin, velvety to the touch, and hanging loosely down at the side of the head. The neck is long, and the skin loose, with prominent breast-bone, but a deep chest.

1 At one time Mr. Tottie of Bell-Busk had the finest kennel of Bassetts in the kingdom. 


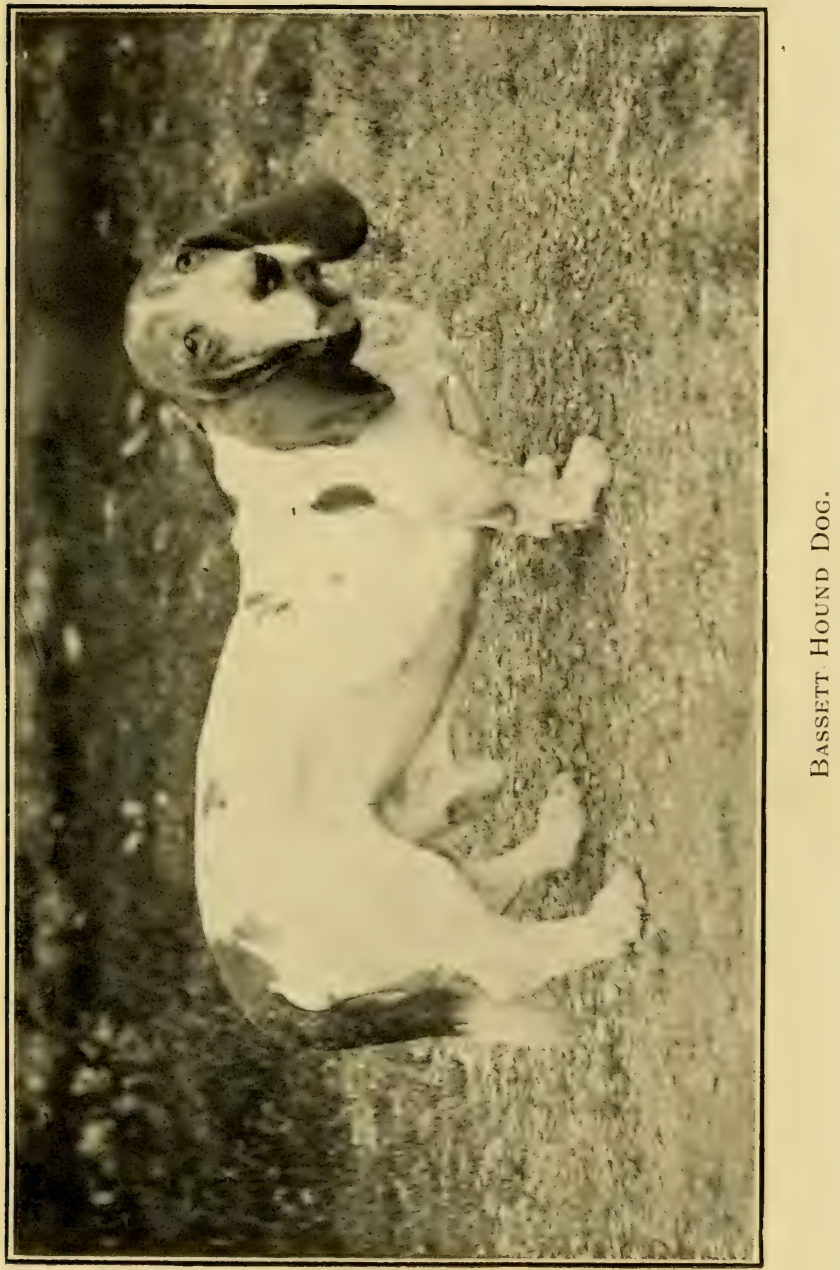





\section{POINTS OF THE VARIOUS BREEDS 55}

The strength of shoulders and arms in a typical Bassett is remarkable. The fore-limbs are short, and turn inwards at the knees, then outwards; in fact, a Bassett to be typical must have a good crook. The back and the loins are long and strong, the longer the better, provided that the hound is well coupled. Tail long and thick, and carried down. The coat must be smooth and close, but there is a rough-coated variety. Judges pay a great deal of attention to general symmetry; to the correct conformation of the head; and, above all, to the facial expression. The bloodhound features of the head, face, and ears are less displayed upon the rough-coated variety, whose facial expression is not altogether unlike that of the otter-hound. During the last few years the Bassett classes at the Palace Show have been somewhat poorly filled, but in 1906 Her Majesty the Queen showed a smooth-coated bitch, 'Sandringham Dido,' bred by Colonel Annand, and with it, we are pleased to note, obtained two first prizes. The rough-coated hounds have never been as much patronised as the smooth, yet they are very handsome dogs.

\section{The Greyhound.}

It is hardly necessary to enter into a detailed discussion regarding the uses and past history 
of the Greyhound; in fact, it is impossible to do so, owing to the limited space at the disposal of the writer. According to Hollingshead, the breed was first introduced into Britain during the third century, and Arrian, writing in his Cuinegetticus about A.D. 150, refers to coursing. Only the nobility were allowed to own greyhounds in olden times, and the destruction of one of them was punishable by death. Lord Orford founded a coursing club in Norfolk during 1776. Norfolk has always been famous for its greyhounds, and there is more coursing in this county than in any other in Great Britain. The head of a greyhound is long and narrow, widening towards the eyes; in fact, the skull should be fairly wide. The nostrils are long, and the nose broad; but a long lean head is regarded as typical. Eyes bright, with a far-reaching expression. The ears should be small, and carried close to the side of the head, but raised during attention. The neck must be long, possess a great degree of flexibility, and end in oblique shoulders. The shoulders should not be overloaded with muscle, or the dog is hampered in its movements. Breast ought not to be wide, but great depth of chest is of prime importance, any shallowness in this region being decidedly objectionable. The back and the loins are long, but special development of the 


\section{POINTS OF THE VARIOUS BREEDS 57}

muscles of the loins is very necessary. An arched back is preferable. The tail is long, nicely curved, and free from any coarse hair. Breeders of greyhounds necessarily pay a great deal of attention to quality in the regions of the fore and hind limbs. Arms and fore-arms must be well muscled, clean in outline, and of good length, ending in oblique pasterns and compact feet. The first and second thighs are longer in the greyhound than in any other variety of dog, and are endowed with a high degree of muscular development - an indispensable factor in the coursing dog. The gaskins should be long; the hocks clean, and strong; pasterns long, and feet compact. In short, a greyhound must be built upon exactly the same lines as the thoroughbred or race horse, in which speed and endurance, for a given time, are developed to the highest degree of perfection.

\section{The Pointer.}

Now that 'driving game' is extensively practised, the pointer is not in such demand as it was before this method was introduced for bringing birds to the guns. The same may be said of the setter, the retriever having to some extent displaced these splendid gun-dogs. There is a considerable amount of evidence to 
show that the pointer has been derived from the Spanish pointer, a variety of dog that was larger, slower at its work, and less even-tempered than the present variety. The most popular are white dogs with liver-coloured or liver-and-white ticked markings, but judges are not too particular about the colour of the markings of the dog, provided that it is excellent in other points. Lemon-and-red markings are frequent, whereas all liver-coloured, red, or black pointers are occasionally met with, the last-named the least frequently. A medium-sized dog is preferred, and bitches are usually somewhat smaller than dogs, and of a slightly lighter build. Here is a description of the typical pointer:-Head of medium length, fairly wide in the forehead, with a squarish outline of muzzle and broad nostrils, the colour of the last-named corresponding with the markings of the dog, except in the case of a lemon-marked dog, in which the nose is fleshcoloured. The eyes small, deep hazel, and penetrating. The muzzle is deep, and the cheeks hang slightly below the level of the jaw; the ears should be thin, clothed with fine soft hair, and perfectly free from any fringe. The neck is rather long, this being necessary for their work; the breast of medium width, the chest deep. The shoulders slope gracefully, and the 
elbows are carried close to the sides. A pointer for the show-ring must excel in neck, shoulders, withers, and fore-limbs. If the chest is not well let down, too much daylight will be seen, especially when the dog is viewed from the front; the fore-arms must be clean and straight, big-boned, and the pasterns long, of graceful slope, ending in strong-arched toes. Back and loins of medium length, but powerful, ending in a gracefully carried, neatly set-on stern. The pointer-judge is very keen on quality in this region. The stern should be carried straight out, the contour of a pointer, from head to stern, forming a striking contrast to that of every other variety of dog, unless it be the setter. The quarters should have a graceful slope, and the thighs the same. The hindpasterns to be of proportionate length, well rounded, ending above in strong hocks. Both at the walk and the trot a pointer should exhibit graceful and lively movements, its action being free and fast. So far no mention has been made of temperament, which is most important. A pointer should have a sweet temper and a high degree of intelligence, otherwise it will never be a clever worker. Any one contemplating the purchase of one of these dogs should either buy a puppy and have it broken by 
an expert keeper, or else purchase a thoroughly broken animal, but this he ought never to do until a reasonable trial has been allowed. At the beginning of the shooting season many sportsmen arrange for the hire of pointers, setters, and retrievers, but many of the firstnamed would hardly be recognised as such, unless the fact were pointed out to one.

\section{The Setter.}

Setters are divided into English, Irish, and Gordon or black-and-tan setters, but the English, sometimes spoken of as the Laverack setter, has always been the most popular variety, and the Irish setter in greater demand than the Gordon. All the setters have, however, suffered as a variety of dog since the introduction of driving the birds to the guns. The most famous strains of English setter are those known as the Laverack, the Blue Belton, and the Llewelyn. The whole of the setters are evidently of remote origin, though there is nothing definite known as to how the breed has been derived, but it is generally believed that there is a good deal of the spaniel element in it; in fact, the Sportsman's Cabinet refers to the setter as the 'rough' spaniel, and to the pointer as the 'smooth' spaniel; hence the term 'setting' or 'improved' 


\section{POINTS OF THE VARIOUS BREEDS 61}

spaniel is sometimes applied to this breed. In a monograph on the setter, written by $\mathrm{Mr}$. Laverack, the writer gives it as his opinion that the setter is older than the pointer, and that it has been derived from the spaniel, an opinion that is entitled to respect. The Gordon setter, in all probability, was produced by crossing a black-and-tan collie with a setter, a theory supported by the defective carriage so commonly seen amongst Gordon setters, and by the socalled 'tea-pot' tail. The 'red' or Irish setter' is also regarded as having spaniel blood in its veins, but little of a definite nature is known of its origin. All setters have unquestionably had a common origin, the Irish and the Gordon being merely variations from the original stock. The leading features of the setter are his dashing style, beauty of conformation, and wonderful physical power. A thoroughly broken setter must find his birds with great alacrity, and any dog that fails in field trials to wind his birds quickly or makes false points, will not stand much chance of being in the money. The colours of the English setter are black and white with tan markings, orange and white, black and white, liver and white, black and white ticked; but blue and white, or blue and white ticked, are the most fashionable colours. 
The Irish setter must be a deep red, and the Gordon setter, of a slightly heavier build, a raven black, with tan markings upon the limbs, etc. There is an elegance about the setter not observable in any other variety of dog. The head of a typical setter should be long and narrow, and the occipital dome rather high, with a deep muzzle square in outline, ending in broad nostrils; ears carried close to cheek, thin, and covered by soft hair; eyes of medium size, and deep brown in colour. A deep chest, clean oblique shoulders, and a strong neck of medium length, with oblique carriage of the head, are essential points of beauty. There must not be too much evidence of stop, otherwise the dog becomes what is called 'dish-faced,' a conformation to which the setter seems predisposed.

The fore-limbs should be perfectly straight; the arms muscular; and knees and pasterns broad, an oblique position of the pasterns being the best conformation. Feet well-feathered between the toes, resembling those of a hare. Back and loins strong, ribs well sprung, flank short, and stern carried in a line with the body. Judges pay a great deal of attention to character in this region. The tail is well-feathered below, the hair being longest towards the base and gradually tapering as the tip is approached. 
This is spoken of as the 'flag,' and it must be carried in line with, or slightly above the level of, the back. The carriage of the tail gives the true setter character, and any inclination towards an upward curl is, for the show-bench, distinctly detrimental. The hair upon the tail must be silky and free from curl. The hindquarters should be beautifully moulded, indicating power and freedom of action, and be elegant to a degree. The weight for a setter ranges from 50 to $65 \mathrm{lbs}$, when in good condition, slightly less for bitches. The coat must be long, flat, dense, and silky in texture, without wave or curl. When a setter moves, every action should show dash and style, and perfect harmony of the various parts of the body. The constitution of the setter varies, especially if dogs have been a lot inbred. Remarkably high prices have been paid for field-trial winners, and a thoroughly broken setter will always command a good price, provided that it shows good quality throughout.

\section{Retrievers.}

Retrievers are divided into flat-coated (formerly spoken of as wavy), curly-coated, and Labradors. Within the last few years the retriever has become extremely popular; in fact, there are far more retrievers in use now than at any other 
period in their history. At one time many sportsmen used to train their pointers and setters to retrieve, and in the United States this is still a general custom. Although handy to have your birds found and retrieved when shot, it is detrimental to both the pointer and setter to retrieve. The retriever is not an old variety of $d o g$, and no doubt contains a good deal of the Newfoundland element in his blood, as will be seen in many inferior specimens. We do not mean inferior in work, for many of these large, rough-coated retrievers are really splendid field-dogs, but the majority of keepers have now gone in for first-class strains of retrievers, more especially since the foundation of the Gamekeepers' Association, and its annual Show, which does so much towards fostering a feeling of goodwill between master and servant, because the latter takes a much keener interest in breaking and training a dog belonging to himself than he would take if the dog belonged to anybody else. It is within the last twenty or five-and-twenty years that the flatcoated retriever has attained such a high degree of perfection, chiefly through the influence of a small band of specialists in the breed. The flatcoats and the Labradors are in the most demand, because it is not at all an easy matter to obtain tip-top specimens of the curly-coats, or, if one is 


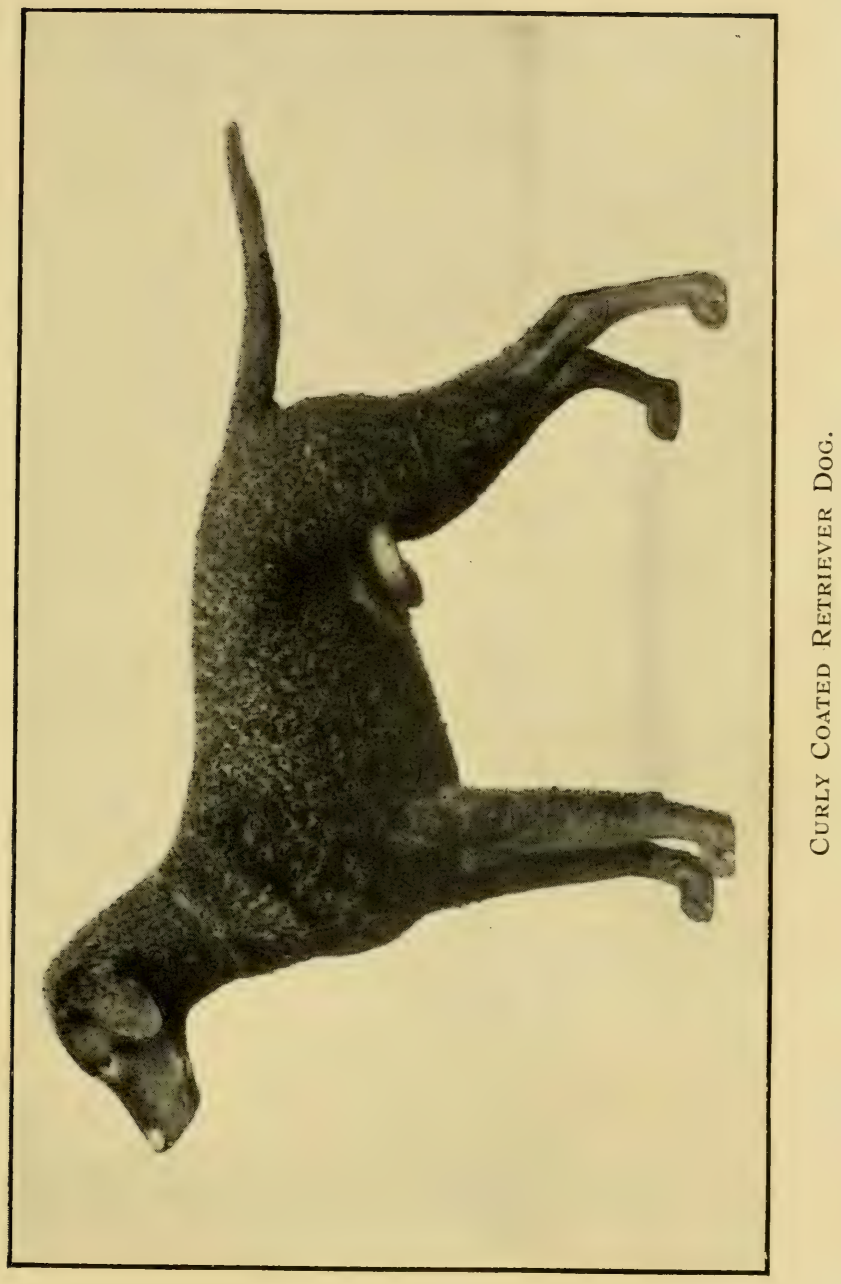



obtained, to keep the coat even in moderate condition. There is a vast difference between the 'show' form and the 'working' form of the curlycoated retriever, but a flat-coated one need not give much trouble in this respect. In some retrievers one can detect a trace of setter blood, no doubt introduced for the purposes of improving the carriage of the stern, and for increasing the dash, working pace, style, etc. This, however, can only exist to a very trifling extent, otherwise the utility of the retriever would be interfered with. A good retriever should work without any slip, and most gamekeepers, retriever-breakers, etc., now train their dogs to do without it, and this is the correct plan. First and foremost amongst all qualifications stands that of temperament: a retriever ought to have a sweet temper, to be obedient, responding, when broken, with alacrity, to his master's commands. Another important qualification is a keen scent. Any one wishing to see really clever working dogs congregated together ought to visit the autumn trials of the Retriever Society. If a sportsman wishes to purchase a thoroughly broken retriever, the writer advises him to apply to the Secretary of the Gamekeepers' Association, Hertford, or to consult the advertisement columns of the Gamekeeper's Gazette, published on the first of every month at Hertford. 
Therein he will find dogs of all grades, but, if he is willing to pay a fair price, there should be no trouble in securing a first-class dog. In every case it is advisable to have the animal on trial for a week or two. The points of the flat-coated retriever are as follows:-

Coat must be of raven blackness, perfectly flat, dense, of medium texture, and free from any sign of wave or curl; but it must be understood that the so-called 'wavy' coat offers no detriment so far as the dog's working capacity is concerned. A flat coat is the orthodox one for show purposes, and any tendency towards openness of coat is distinctly faulty. A moderate amount of feather at the backs of the fore-limbs and down to the hocks in the hind-limbs is desirable. A great deal of attention is paid to quality in the regions of the head and stern, and to the general conformation of the body. The ears should be small, fine in the leather, covered by soft, silky hair, and carried close to the side of the head; the colour of the eyes is most important, and a judge will not award a retriever a prize if it has light eyes, an extremely common fault. The iris must be of a deep hazel, but in order that the reader may fully appreciate the correct colour of a retriever's eyes he should visit some show, and thus obtain practical information. The nostrils ought to be broad, the head narrow, 


\section{POINTS OF THE VARIOUS BREEDS 67}

but only so in proportion to dog's size. A retriever has a slightly-domed skull, and a very benevolent expression upon the face. The neck is of medium length, and the chest fairly broad and deep. The back and the loins are strong, and the stern is carried slightly below, or on a level with, the back, but it must never curl upwards, or over the latter, this being one of the worst faults a retriever can possess, so far as show-bench points are concerned. Fore-limbs must be straight, powerfully built, and end in close, compact feet, well-feathered between the toes. The carriage of the head is important; this should be inclined to be lofty, enabling the dog to carry his game with greater ease. A sportsman will say that as yet we have not mentioned what is to him the most important point in a working retriever, the mouth. A 'hard-mouthed' retriever will never command a good price. A 'tender' mouth is one of the most important qualities, but of course neither of these can be distinguished on the show-bench. The hind-quarters, thighs, and the conformation of the hocks and pasterns must be good. Some retrievers are inclined to be cow-hocked, and others weedy, either fore or aft. Liver-coloured specimens with flat coats are nothing like so common as the wavy-coated, whilst typical specimens of the curly variety are somewhat difficult 
to obtain. No matter whether it be a black or liver-coloured retriever, the presence of white hair upon any part of the body is detrimental for the show-bench, unless it be a trifling amount of white hair upon the breast, though judges prefer a dog absolutely free from white. The points of the curly-coated retriever are practically identical with the flat, except that the curlies are a trifle longer in the limb, and slightly different in the skull. A curly-coated retriever ought to have a dense mass of short, sharply-defined, crisp, tightlycurled ringlets, but commonly the coat or a part of it lacks these qualities. For instance, the curls may be orthodox upon the ribs, quarters, etc., but slack on the back, or vice versa ; openness of curl has a tendency to show itself towards the stern, and over the ribs.

The Labrador retriever is a heavily-built dog, the head and expression upon the face being much heavier than in the flat-coated retriever. The typical colour is black, but, strange to relate, lemon-coloured specimens, more rarely white ones, now and again make their appearance in a litter bred entirely from black parents. A strain bred by Lord Tweedmouth was of the first-named variety. The occasional appearance of these pups in litters may be looked upon as instances of atavism, i.e. harking back to some antecedent 
generation, and it may not be out of place to mention the Chesapeake Bay Dog, an American variety closely allied to our retriever, which has a reddish-brown or brownish-red coat, with hair from a half to an inch and a half in length, dense and wavy (see Foreign Dogs). The coat of the Labrador must be flat, hard, and dense; the back, loins, and quarters particularly strong, and the tail carried straight out. The eyes must be dark, ears thin, and covered by soft hair, and the body stoutly built throughout, any tendency towards weediness of body or limbs being faulty. All varieties of retrievers are hardy, consequently puppies are not difficult to rear, and pedigree pups can be obtained for two or three guineas, and a soundly-broken second or third season dog ought to be got for twelve to five-and-twenty pounds, though several hundreds have been paid for dogs of exceptional brilliancy, either in the field or on the show-bench.

\section{Spaniels.}

The different varieties of Spaniels have always been great favourites with sportsmen, and there is evidence to show that the breed has been used by the gunner for several hundreds of years, and its popularity is now greater than ever. It has been a particular favourite with the single-handed 


\section{THE DOG}

sportsman, and those who are fortunate enough to become possessed of a really cleverly-broken dog will find the spaniel hard to beat. Most of them are good-tempered, make excellent companions in the house or field, are obedient, of hardy constitution, and there is not much trouble experienced in breaking them when derived from a working strain. There are several varieties, which are as follows :-
The Irish Water Spaniel.
The Water Spaniel.
The Clumber.
The Field Spaniel.
The Sussex Spaniel.
The Cocker Spaniel.
The Welsh Spaniel or Springer.

The reader who is about to purchase a spaniel for sporting purposes will naturally ask which variety ought he to select. To answer this question in a thoroughly satisfactory manner is somewhat difficult, and the intending purchaser must first of all consider the class of sporting he is going to take up. For instance, if he requires a dog principally for hunting and retrieving wildfowl, the Irish water spaniel can be recommended in preference to all others. For land shooting and hunting coverts, etc., the Clumber is admirable, and these dogs are rapidly coming to the 


\section{POINTS OF THE VARIOUS BREEDS 71}

front, although they have always been held in high esteem as 'gun' dogs. The Cocker spaniel is a merry little worker, and a brace will perform an extraordinary amount of work, their diminutive size giving them an advantage over larger dogs. The Sussex spaniel, when pure bred, cannot be regarded as being of any particular value for the sportsman; doubtless breeders of this variety will take exception to the author's remarks, but every man ought to be guided by his own experience, whether it be general or not.

Exhibition field spaniels and Sussex spaniels are nearly always very short upon the leg, and this is detrimental for work, more especially amongst the furrows. Of course the term 'field' spaniel is applicable to any variety of spaniel capable of adapting itself to, or being employed in connection with, the hunting and retrieving of small game. Most of the field spaniels on the show-benches to-day have more than a trace of Sussex blood in them, which is to be regretted; and the sooner this is eliminated from the field spaniel the more useful the breed will become. Any tendency towards heaviness of body, shortness of limb, and sluggishness of temperament, renders a spaniel of less utility for work.

If it is desirable to purchase a spaniel already 
trained to the gun, the reader cannot do better than consult the columns of the Gamekeeper's Gazette, published on the first of every month, and obtained from the editors, Messrs Gilbertson and Page, Hertford. This paper circulates largely amongst gamekeepers, and some really excellent working spaniels can usually be procured through its columns. A description of the chief varieties of the spaniels is as follows :-

\section{The Irish Water Spaniel:}

When thoroughly broken, these dogs are capital workers, for either fur or feather. They are smart, active-looking dogs, hardy in constitution, intelligent, and not a great deal of trouble to break; in fact, retrieving from water is practically inbred in them. They are much larger than any other variety of spaniel, being about 20 inches at the shoulder. For some reason or other very few sportsmen and gamekeepers outside Ireland have kept these dogs, but in Ireland they have always occupied the front rank as a sportsman's dog, their utility being well known. There are two clubs watching over the interests of the breed:-The Irish Water Spaniel Club and the Sporting Irish Water Spaniel Club. The following is the scale of points framed by this body :- 


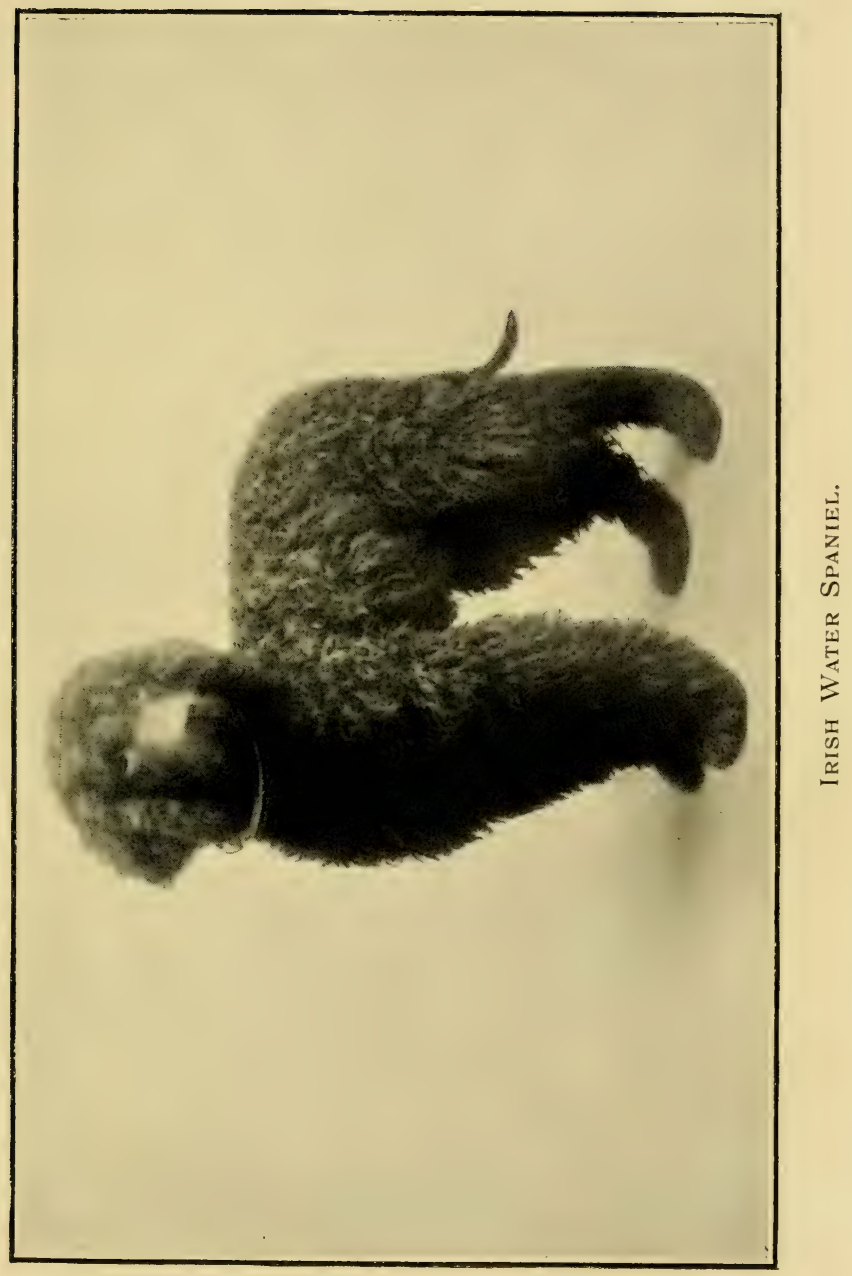





\section{POINTS OF THE VARIOUS BREEDS 73}

The Sporting Irish Water Spaniel Club.

\section{Scale of Points.}

Positive Points.

Head and jaws, eyes and

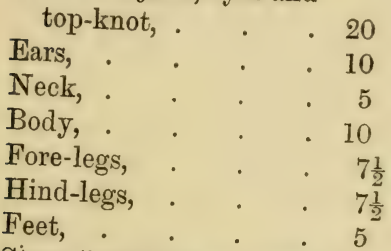

Stern (including carriage), 10

Coat, . . . 15

General appearance, . 10

Total

Negative Points.

White on chest, . . 5

Paleness of coat, . 10

Light eye, . . 10

Woolliness of coat, . $\quad 20$

Feather on stern dis-

qualifies; Feather on

face disqualifies; White

on feet disqualifies ;

Feather on front part of hind-leg from back

to front, . . 10

Want of feather on front

of fore-legs,

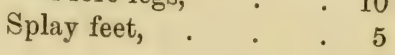

$\begin{array}{r}10 \\ 5 \\ \hline 70\end{array}$

Descriptive Particulars.

HEAD.-Skull and muzzle should be of good size; skull high in dome, good length, and fairly wide, showing large brain capacity; muzzle long, strong, and somewhat square in appearance; face perfectly smooth. Top-knot should consist of long, loose curls, growing down into a welldefined peak between the eyes, and should not be in the form of a wig, i.e. growing straight across.

EyEs.-Comparatively small, dark amber, and very intelligent-looking. 
EARS-Very long and lobe-shaped in the leather, set low, hanging quite close to the cheeks, and covered with long twisted curls of live hair.

NECK.-Fairly long, strong, and arching (carrying the head well above the level of the back), and strongly set into the shoulders.

Body (including size and symmetry).-Fairsized, round, and barrel-shaped; so stout and compact as to convey a 'cobbiness' in appearance. Height-Dogs, about 21 to 23 inches at shoulder; bitches, about 20 to 22 inches (not under).

Nose.-Large and well developed, and of a dark liver colour.

Shoulders and Chest.-Shoulders very powerful but not too straight or 'clody'; chest deep, but not too wide or round between the fore-legs though large in girth, with ribs well-sprung behind the shoulders.

BACK AND LoINs.-Back, short, broad, level, and very powerfully coupled to hind-quarters. Ribs carried well back, and loins deep and wide.

HiNd-QUARTERS.-Very powerful, with long, well-bent stifles, and hocks set low.

STERN.-Short and smooth, strong and thick at root (where it is covered for three or four inches with short curls), and, gradually tapering, 


\section{POINTS OF THE VARIOUS BREEDS 75}

ends in a fine point. It should not be long enough to reach the hock joint, and should be carried nearly level with the back in a straight line.

Feet and Legs.-Feet large, somewhat round and spreading, well clothed with hair, both over and between the toes, but free from any superfluous feather. Fore-legs, well-boned, and straight, with arms well let down, and carrying the fore-arm at elbow and knee in a straight line with point of shoulders.

CoAT._Composed of dense, tight, crisp, ringlets, entirely free from woolliness; the fore-legs covered with feather, which should be abundant all round, though shorter in front, so as only to give a rough appearance; below the hocks, the hind-legs must be smooth in front, but feathered behind down to the feet.

Colour.-A very rich puce liver; white on chest objectionable. Colour of coat to be judged by under coat and not by exterior.

General Appearance.-That of a smart, upstanding, strongly-built, but not leggy dog, combining great intelligence and endurance, with a bold and dashing eagerness of temperament.

\section{The Water Spaniel.}

The term 'water spaniel' is applicable to any 
variety of spaniel used for retrieving from water, and it is only within the last few years that the Kennel Club have seen their way to open up a class for these dogs, which reads 'for water spaniels other than Irish.' The ambiguity of this classification is at once obvious, and there were only two entries at the show in 1906, 'Chorister,' and 'Beechgrove Wildflower,' awarded first and second prize respectively, doubtless without overtaxing the judge's discriminating power. If any one were to ask the question, 'What constitutes a water spaniel ?' he would indeed be a bold man who attempted to define the meaning of the term. Many of the water spaniels shown are certainly of a very workmanlike order, but not of a distinctive type of spaniel, some of them having more than a dash of Irish water spaniel blood in them; in fact, it is more than probable that one could produce a very useful type of dog by crossing an Irish water spaniel with a working strain of field spaniel. The typical colour is liver and white, but there must be no fore-lock like that in the Irish water spaniel. By careful selection this fault might easily be out-bred, if the cross suggested were employed. The legs should be rather long, big in the bone, and the feet well feathered; brown eyes; fairly long ears, clothed with curly hair, the curls to be smaller 


\section{POINTS OF THE VARIOUS BREEDS 77}

than those on the body. The chest must be of good capacity, and the body a medium length, with a coat of a dense curly nature. In the review of a water spaniel the picture conveyed to the mind of the observer should be that of a medium-sized, close-coated, big-boned, wellfeathered, alert, and intelligent-looking dog, capable of undergoing, and willing to undergo, any reasonable amount of exertion.

\section{The Clumber Spaniel.}

This useful variety of dog is said to have originated at Clumber Kennels, and in Daniel's Rural Sports it is stated that the immediate ancestors were given by a French nobleman, the Duc de Noailles, to a Duke of Nerwcastle about a couple of hundred years ago. The Clumber spaniel has always been a favourite with sportsmen, more especially amongst the aristocracy. Colonel Hamilton, in his Recollections, writes: 'A spaniel known as the Clumber breed-his Grace the Duke of Newcastle always shooting over them in his woods-is much sought after by sportsmen.' There is also a coloured print by Bartolozzi after Wheatley with the Duke of Newcastle represented on a bay shooting pony, surrounded by a group of Clumbers. One, William Mansell, at that time had the care of them for thirty years; this was 
about the year 1807. The Clumber spaniel has an excellent nose, and is equally good on land or water. They are powerful swimmers, and useful in any kind of shooting, be it on fen, moor, or loch. Constitutionally the breed is somewhat delicate, and many breeders experience considerable difficulty in rearing their puppies, distemper accounting for a large percentage of losses. In point of build the Clumber is the most massive of the spaniels, and it is his enormous strength that enables him to be such an untiring worker. The typical colour is lemon and white, or varying shades of orange and white, but a judge of Clumber spaniels prefers a dog with as few markings as possible, and a heavily marked Clumber is looked upon with disfavour. Really tip-top specimens are difficult to obtain, but any one who wishes to go in for Clumber breeding should purchase a bitchpup of a fashionable strain and mate her with the best dog he can find. The coat should be dense, straight, and silky, with profuse feathering down the back of the fore and hind limbs; body long with well-sprung ribs, and deep at the chest. The head must be massive in all proportions, but not short, as shortness of head is looked upon as a defect; hence the nose must be long, and the nostrils broad and flesh-coloured. The occipital dome must be fairly well developed, and the 
Clumber always shows good evidence of 'stop.' Eyes, hazel, preferably a dark hazel, the light hazel eye being looked upon as non-typical. Ears of moderate length, set on low, at the same time carried close to head. The fore limbs must be massive, any tendency towards lightness in this region being decidedly defective; the same remark applies to the hind limbs, and there ought to be no feather below the hocks. Feet must be large, strong, well-clothed with hair between the toes, and carried in a line with the body, i.e. turned neither in nor out. Tail never carried above the level of the back, even under excitement. The Clumber spaniel makes a very good companion, owing to its docile disposition; and one of the best companions the writer ever had was that famous Clumber spaniel 'Champion Psyche.'

\section{The Field Spaniel.}

The classification adopted by the Kennel Club for Field spaniels is : of black; other than black; (most of these have been derived, directly or indirectly, from the Sussex spaniel, as any careful observer will be able to note on reference to the show bench); field spaniel; and the Sussex spaniel. Strictly speaking, the field spaniel ought to possess such lines as will enable the dog to give the best account of itself whilst at work 
in the field, but this does not apply to the modern type of field spaniel, the points of which are given as follows:-Colour, either black, black and white, black, white and tan, black and tan, liver, liver and white, roan, liver, roan and tan, etc. Coat, perfectly flat, of medium length, close, glossy, and free from any sign of curl, with a moderate degree of feathering upon the backs of the fore and hind limbs. Ears, long, heavily feathered; in fact, length of leather and feather upon a field spaniel is a show-bench point. Eyes, according to colour of coat, and the less evidence of the so-called 'haw' the better. The late Mr. Joseph Royle used to show a black field spaniel called 'Zulu,' which did an ezormous amount of winning, but his eyes bore a strong resemblance to those of the bloodhound. This was derived from his Sussex sire 'Champion Bachelor,' and field spaniel breeders are anxious to delete these traces of Sussex blood. The body must be long and low ; the head long, but not have a heavy facial expression; fore and hind limbs short, big-boned, and perfectly straight. Some field spaniels are too short in head, others too long and narrow in the muzzle, whilst many are defective in coat, too short in the body, short in ears, leggy, or weedy in other respects. These remarks are, of course, solely applicable to the show-bench spaniel. 


\section{POINTS OF THE VARIOUS BREEDS 81}

\section{The Sussex Spaniel.}

This variety of spaniel, it is said, had its origin at Rose Hill in Sussex, and it used to be customary five-and-twenty or thirty years ago for connoisseurs to scour the county of Sussex for specimens of the breed. Most of the present Sussex spaniels have been derived from a cross with the black spaniel, this cross being started by Jacobs. A Sussex spaniel ought to be of a rich golden liver colour, very long in the body, and low on the leg; have long, heavily feathered ears, and big-boned fore and hind limbs, the backs of the fore-limbs being well feathered to the ground, but those of the hind only to the hocks. The head is large, and the 'stop' fairly well defined. Stern to be carried horizontally or slightly below the level of the back. The Sussex spaniel is usually a good-tempered dog, and its constitution tolerably good, though weakened by so much inter-breeding, which has had to be indulged in owing to the limited number of specimens. It is not a variety of spaniel the author would recommend any one to take up either for work or profit, the Clumber being vastly superior to it in every respect. - Most sportsmen will endorse this. 


\section{The Cocker Spaniel.}

This is a delightful type of spaniel, useful for both companionship and sport, and it exists in a variety of colours, such as black, red, liver, roan, etc. The Kennel Club classifies for 'black' and 'other than black' Cockers. In all probability the Cocker is one of the oldest varieties of spaniel, being referred to by Dr. John Caius as far back as 1576. To be typical a Cocker spaniel dog ought not to exceed five-and-twenty pounds in weight, a bitch being a few pounds less. The head should be of medium length, but not as heary as in the field spaniel. Any tendency towards the so-called 'snipy' muzzle is a defect-a very common one. Eyes, dark colour, but in accordance with colour of coat, i.e. either black or brown. The body should be long, the coat flat and abundant, of silky texture. A wary coat is general, but a flat coat is preferred for the show-bench. Neck of medium length; shoulders strong; chest deep; ribs well-sprung; back and loins strong; flank and the belly full; stern set on low; and the fore and hind limbs well feathered, ending in medium-sized feet, having plenty of feather between the toes. As in most other spaniels, the ears should be long, set on low down, and have an abundance of fine hair and 
fringe, the length of which it is easy to measure by drawing the free ends towards the nose. The general appearance of a Cocker spaniel should be that of a merry, active, little dog, without the slightest trace of the massiveness of the Clumber, or the slowness of the Sussex. Cocker spaniels are easily reared, and good specimens are not difficult to dispose of; and any sportsman in search of a neat, active spaniel, suitable for indoor residence, cannot do better than purchase one of these, provided that it comes from a working strain.

\section{The Welsh Springer Spaniel.}

Within this last few years the Welsh Springer has rapidly come to the front, and at the Crystal Palace show there is always a good entry of these dogs. The typical colour for a Welsh springer is red and white, or orange and white, but the firstnamed is regarded as the correct colour. The springer is to be distinguished by its size, invariable colour, and shape of ears. The coat must be straight and thick, the head long and moderately broad, with medium length of jaws and nose, the nostrils being flesh-coloured. Compared with those of other spaniels the ears are small. Body of medium length, and chest deep. The legs straight, but not too much feather upon them, whilst the stern must never rise above the level 
of the back. With regard to the eyes, there must be no 'haw' showing, and the colour should be dark brown or else hazel. Welsh springers must not be too short on the leg, nor yet what might be termed 'leggy,' medium length of leg being the correct type. It is an active spaniel, and, if properly broken, makes an excellent gun-dog, its general build being favourable for sport.

\section{The English Springer Spaniel.}

Although the term is somewhat vague, the Kennel Club have classes for English Springers other than Clumber, Sussex, or Field spaniels, and these classes have at recent shows been tolerably well filled. In reality any cross-bred spaniel can go into the English springer class, and this is the reason why one finds such an assortment of coloured dogs in it, such as liver and white, black and white, liver and tan, liver, white, and tan, and so on. The head should be like that of the Welsh springer, and the eyes of medium size; ears not too long. Legs of medium length, without much feather, and the coat thick and wary. The English springer may be regarded as representing the best type of the sportsman's spaniel, provided that it is properly handled in its youth, and it is from the ranks of English and Welsh springers that one may hope to produce dogs that 


\section{POINTS OF THE VARIOUS BREEDS 85}

will give the best account of themselves in field trials and other work.

\section{The Collie.}

The collie has always been an extremely popular variety in almost all parts of the world, and there are very few dog-shows in which the classes for collies are not well filled. It is a breed which has been in existence for many generations, but it is during the last thirty years that it has attained its present high standard of excellence. The origin of the breed is involved in obscurity, but it is generally thought that it has been derived from some species of wild dog inhabiting mountainous districts. There are rough and smooth-coated varieties, also the Old English or bob-tailed sheepdog, but the first-named is that which has always been the most popular, as typical smooth-coated specimens are more troublesome to breed. The collie exists in a variety of colours, and at one time black and tan, or black, white, and tan, the last named being termed tri-coloured, were in great demand, but the sable seems to be the most sought after now. The temperament of the collie is variable, some being extremely docile, others quarrelsome to a degree. Although they make excellent companions and are useful as house dogs, their restless disposition is, in the 
writer's opinion, rather objectionable. For the shepherd a well-broken collie is his right-hand companion, and the sheep-dog trials held in various parts of the country have done a good deal towards improving the working qualities of many of these dogs, but the working collie is not, as a rule-there are numerous exceptions-capable of giving much of an account of himself upon the show-bench. The constitution of the sheep-dog is hardy, and there is not much trouble in rearing pups. There are the English and the Scottish Collie Clubs, and as the standard of points given by these bodies necessarily embraces all the principal features of the breed, the writer has decided to set out these points here.

The following is the English Club's standard of points :-

Srull.-Should be flat, moderately wide between the ears, gradually tapering to the eyes. There ought to be a slight depression at 'stop.' The width of the skull necessarily depends upon the combined length of the skull and muzzle, and the whole must be considered in connection with the size of the dog. The cheek should not be full or prominent.

MuzzLE.-This ought to be of fair length, tapering to the nose, and must not show weakness, be snipy or lippy. Nose must be black. 


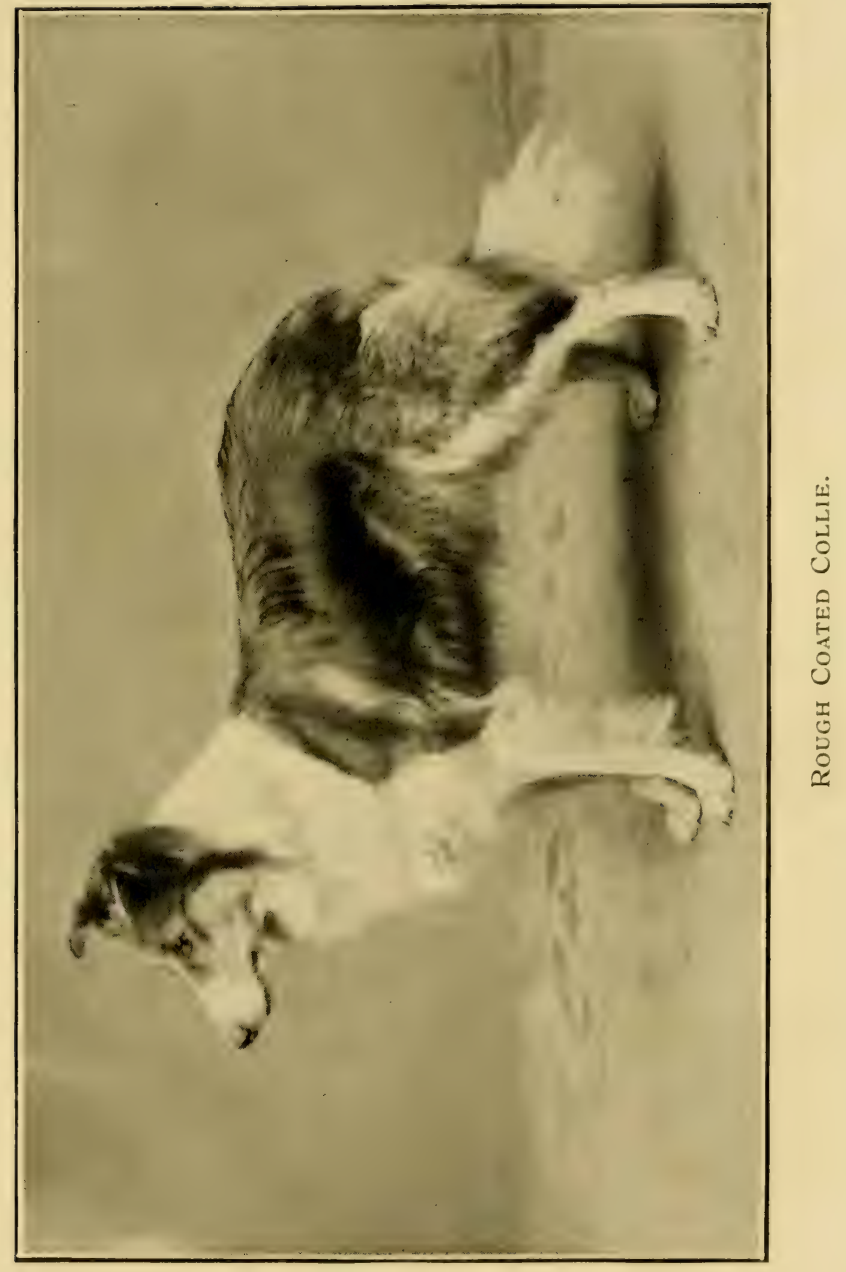



TEETH.-Sound, level, and of good size.

JAws._Clean cut and powerful.

EYES.-Very important; to be of medium size; almond-shaped and brown; merles excepted; same as Scottish Club in latter.

EARs.-These should be small, moderately wide at the base, and well placed on the head. When in repose they ought to be thrown back, brought forwards when dog is on the alert and carried semi-erect, with the tip slightly drooping in an attitude of listening.

NECK.-Powerful, fair length, arched.

BoDy.-To be rather long, well-sprung ribs, deep chest, sloping shoulders, slightly arched loins. Dog must be straight in front.

Fore-Lrmbs.-Straight and muscular, neither in nor out at elbows, with a fair amount of bone. The fore-arm fleshy, the pasterns showing flexibility without weakness.

Hind-Legs.- These ought to be muscular at the thighs, clean and sinewy below the hocks, with well-bent stifles.

FeEt. - These should be oval in shape, toes arched and close together. The hind feet less arched, hocks well let down and strong.

BRUSH.-Tail of moderate length, and carried low, with a slight upward 'swirl' when dog is at 
ease. To be carried gaily under excitement, but never over back.

CoAT.-This should be very dense, the outer coat harsh to touch, and the under one soft, and very close and furry. Mane and frill to be abundant, and hair on brush very profuse. Face smooth, likewise ears at tips, with more hair towards their bases. Fore-limbs, slightly feathered, and the hind-legs above hocks profusely so.

\section{CoLour.-Unimportant.}

Size.-Dogs 22 to 24 inches; bitches, 20 to 22 inches.

Weight.-Dogs 45 to 65 lb., bitches, 40 to $55 \mathrm{lb}$.

N.B.-Note the difference of $5 \mathrm{lb}$. (in both sexes) greater in the English standard as compared with the Scottish.

FAulTs.-Domed skull, cow-hocks, under- or over-shot mouth, curly or soft coat, tail twisted or carried over back, high peaked occipital bone, hare feet, snipy muzzle, crooked legs, weak jaws, light eyes, prick ears.

The following is the Scottish Club's standard of points :-

HeAD.-Moderately long in proportion to the dog's size, covered with short soft hair. Skull flat, moderately wide between the ears, and gradu- 


\section{POINTS OF THE VARIOTS BREEDS 89}

ally to the eyes. There should be a very slight elevation of the eyebrows and very little stop.

MuzzLe.-To be of fair length, tapering to the nose, which, whatever the colour of the dog, should be black. The teeth ought not to be overor under-shot. Both are faults, the last-named the greater.

Eyes.-To be of fair size, not prominent, placed rather close together and lying obliquely. It is this that gives the fox-like expression. As to colour, the eyes ought to be brown, darker the better. Yellow eyes are a bad fault. Merled dogs to have the so-called 'wall' or 'china' eye or eyes.

EarS._Small, close together at top of head. At rest to be buried in the frill, other times semierect.

NEcK.-Long, arched, strong.

BoDy.-Rather long than short, ribs well rounded, chest deep and narrow in front, but of fair breadth behind the shoulders. Loin, rather long, slightly arched and powerful.

LEGS._Fore-legs straight and muscular, with a fair amount of flat bone; fore-arm moderately fleshy, hind-legs less so, very sinewy and hocks well bent. Pasterns light and long, feet oval, toes well arched and close.

TAIL.-Moderately long, carried low when dog 
is quiet, gaily when excited, and straight out when running.

CoAT.-This is most important. The coat, except on the head and legs, should be abundant, the outer coat harsh to the touch, the inner coat soft and furry, very close-so close that when the hair is parted it is difficult to see the skin. The hair around the neck and chest to be very abundant, constituting the 'frill.' The mark is smooth, the fore-legs slightly feathered and the hind-legs below the hocks smooth. Hair on the tail very profuse, and on the hips long and bushy.

Colour.-Unimportant. Sable and white dogs are very fashionable.

Size.-Dogs, 21 to 14 inches at shoulder, and bitches a couple of inches less.

Weight.-Dogs, 45 to 60 lbs.; bitches, 40 to $50 \mathrm{lbs}$.

General Appearance.-A lithe, active dog, with no useless material about him, his deep chest showing strength, his sloping shoulders and wellbent hocks and his 'bawsint' face, high intelligence. The face ought to bear a sharp, doubtful expression. As a whole, he should present an elegant and pleasing outline, quite distinct from any other of our domesticated families, and show great strength, activity, and endurance.

FAults.-Domed skull, heavy pendulous ears, 



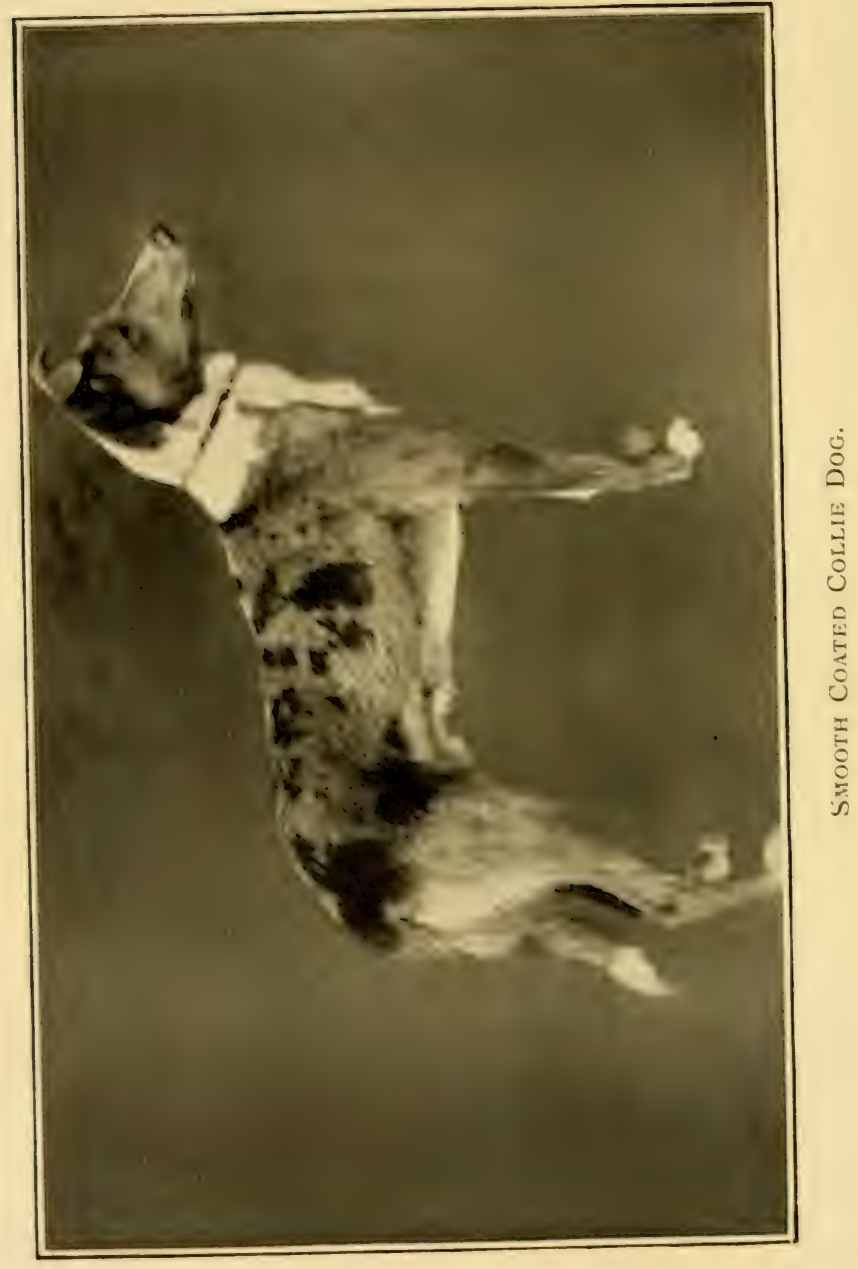


heavily feathered legs, short tail, full soft eyes, high peaked skull

Scale of Points.

Value Value

Head, . . . 15 Legs and Feet, . . 15

Eyes, . . . 5 Tail, . . . 5

Ears, . . . 10 Coat, . . 20

Neck and Shoulders, . 10 Size and General Feat-

Body, . . . 10 ures, . . . 10

50

Grand Total, . . . . . 100

\section{The Smooth-Coated Collie.}

In most respects the points of the smoothcoated collie are practically identical with those already given; the coat must be fine, but very dense, especially the undercoat; and there must be a very small amount of feather on the tail and hind-quarters. What is termed a 'blue merle,' that is, a harlequin collie, is most frequently met with in the smooth coats, and the eyes must correspond, i.e. the dog must have the so-called 'china' or wall eye, which implies that the iris is destitute of pigment either in one or both eyes.

\section{The Old English or Bob-Tailed Sheep Dog.}

These are extremely handsome dogs, and make 
first-class companions, whilst for the shepherd they are even superior to the ordinary collie. They are of an extremely hardy nature, and superabundance of hair upon the body renders them particularly suitable for resisting cold and wet. The term 'Smithfield' or 'Drover's dog' is sometimes applied to them. Any one in search of a really first-class companion ought to buy one of these dogs, the prices of which range from thirty shillings up to five-and-twenty and thirty pounds, but an excellent puppy can be purchased for about three guineas. It is a remarkable fact that an English sheep-dog with a long tail, occasionally makes its appearance in a litter of bob-tails. The colour most sought after is some shade of blue or gray, with white markings, but there must not be any trace of yellow or brown amongst the blue hairs. In many specimens the white predominates, but gray, grizzle, blue, or blue merle, with or without white markings, are typical colours. Judges pay a great deal of attention to the coat; in fact, if a bob-tail is not good in this respect, he will never be any use for show purposes. The coat must be abundant, hard in texture, and shaggy, but any tendency towards a curly coat is distinctly objectionable. The undercoat must be very dense and waterproof. The typical height 


\section{POINTS OF THE VARIOUS BREEDS 93}

(shoulder measurement) is about 22 inches, though bitches usually measure about 20 inches. The head ought to be squarish, the skull being broad, and domed, so that there is a distinct evidence of stop. The hair on the forehead should be long and shaggy; in fact, the eyes are largely obscured by this. Nose of medium length, with long hair from either side, ending in broad nostrils, black in colour. The eyes differ in their colour, in accordance with the shade of the dog, but in the blue merle, the china eye is the correct one. Ears must be small and carried close to the side of the head; neck short and stout, ending below in broad withers; strong shoulders, arms, and forearms, all of which should be covered with a superabundance of shaggy hair. These dogs stand lower at the shoulders than behind, which is a characteristic of the breed. Feet small and round, and the pads hard. The body must be compact, and is short in proportion to the size of the dog. The front ribs are deep, which gives the chest great capacity. The loins must be arched, well-rounded towards the stern, and from the quarters down the thighs there must be an abundance of shaggy hair, thick and soft beneath. Taken all round, the old English sheep-dog has a very bear-like appearance. His movements 
are extremely active, and he has an intelligence surpassed by no other breed.

\section{The Dalmatian or Carriage Dog.}

At one time no equipage was considered complete without one of these dogs, but they are not now as fashionable as they used to be. The Dalmatian and the horse have been depicted by numerous artists, both in and out of the stable, and when one sees this animal fulfilling some other duty, say acting as a yard dog, he feels that the Dalmatian is no longer required, its natural avocation having become an affair of the past.

At a recent Kennel Club Show there were only three entries in the open dog class and four entries in the open bitch class, thus showing the comparative scarcity of the breed, although there is a club specially devoted to it, and the Ladies' Branch of the Kennel Club and the Ladies' Kennel Association offer special prizes. There is a possibility of its revival, but this is somewhat remote. The Dalmatian is built upon foxhound lines, being a smooth-coated, short-haired, big-boned, clean-jointed, deep-chested, cleanlimbed dog, especially adapted for running. The body is entirely white, with evenly distributed spots, which are black, or sometimes chocolate. 
The even distribution of spots and their uniformity of size are most important points, and, if a dog is badly marked, it is severely handicapped in the show-ring. Many Dalmatians are defective in this respect, some being too heavily marked, others too lightly marked. Confluence of markings constitutes a serious fault, but a very difficult one to contend with in breeding. The spots on the head, face, ears, and tail are usually smaller than those upon other parts of the body. The coat must be short and thick; tail well spotted, and carried straight down at repose, but straight out during exercise, the hound-carriage of tail being very faulty. The average weight is about 50 lbs. Back and loins to be strong; first and second thighs well clothed with muscle, of good shape, and the hocks turned neither inwards nor outwards, but directly in a line with the body. A Dalmatian must have good forelimbs, but bitches are lighter in the limbs than dogs, and usually weigh a few pounds less. Any tendency towards being undersized is fatal in the show-ring, or for the purposes for which the breed has been designed. The forearms are of medium length; the feet cat-like and strong, but, above all, the pads of the feet must be hard, the degree of hardness being fostered by the amount of running the dog has to do. The eyes should 
be of medium size, and have a black or brown rim round them to correspond with the colour of the spots. A remarkable feature in connection with the puppies is that they have no spots on them at the time of birth, and that it is two or three weeks before the spots make their appearance. Pigmentation begins upon those parts where the skin is thinnest, and it is about the middle of the second year before the process of spotting has attained its full degree of development.

\section{The Whippet or Race Dog.}

These dogs are built upon the lines of the greyhound, and in all points bear a strong resemblance to that breed. They have been produced, it is said, by crossing a greyhound with a terrier. The chief patrons of these dogs are colliers, etc. The first use of the whippet is for rabbit-coursing, and for racing purposes. Most of the races are of about two hundred yards, and the principal are run under the National Whippet Racing Club rules. The chief points of the whippet are as follows:-Colour unimportant, black, red, brindles, fawn, and blue being common. Head long, the jaws powerful, and the teeth sound and level. The coat fine, the ears small, the so-called 'rose' ears being preferred. Shoulders muscular, but not overloaded. 
The breast must not be wide, but a most important point is 'heart' room, which implies great depth of chest. Another important point is in connection with the loins, which must be broad, and clothed with well-developed muscles. The forearms and pasterns are long, with a corresponding length of arm, and the limbs are placed well forward in relation to the body. The hind-limbs must be long in the first and second thighs, with a good sweep in a backward direction, so as to bring the hocks and pasterns well behind the stifles, this giving the dog the advantages of a long stride. A tapering tail, a fine close coat, round feet, a good temper, and a weight ranging between 16 and $24 \mathrm{lbs}$. when the dog is in good hard condition, are the remaining qualifications. For whippets it is advisable to have woollen clothing in winter, and linen in summer.

\section{The Poodle.}

The poodle has always been a fashionable variety of dog, and still maintains his premier position. Only those who have considerable means are able to keep these animals clipped in the height of fashion, and in its untrimmed state the poodle can hardly be considered a handsome dog, but it is endowed with a large amount of 
intelligence, and it is on this account that the showman often makes use of it. The poodle varies considerably in its size, the smaller varieties being spoken of as 'toys'; these weigh from 6 to $10 \mathrm{lbs}$, but the largest class sometimes weigh from 50 to $60 \mathrm{lbs}$. In addition, there are others averaging about 40 lbs., and a fourth class of 20 lbs.; but the 'heavy' weights of the breed are, in the writer's opinion, the handsomest, an opinion endorsed by the majority of ladies. The Kennel Club's classification is as follows:-Corded; non-corded; black and white non-corded; blue and brown non-corded; non-corded any colour but black and white; non-corded under 15 inches; any colour but black and white non-corded under 15 inches, and corded or non-corded under 15 inches; so that this body ignores weight so far as classification is concerned. The principal colours are black, chocolate, red, blue, fawn, silver grey, and white. The blacks are the commonest, but colour does not carry a great deal of weight, provided that the $d o g$ is up to show-standard. The temperament of the poodle varies considerably, and many known to the writer have been vicious towards strangers, but he has no desire to slander the animal's temperament. If the coat is corded, the cords must be very abundant, closely set, and tight; if curly, it is indispensable that 


\section{POINTS OF THE VARIOUS BREEDS 99}

the curls should be tight and close, in fact the closer the better. The general build of a poodle is that of a powerfully built animal in proportion to its size; that these dogs are strong may be proved by watching them in combat. The head should be long, and the face straight; eyes dark brown, but, if the dog is chocolate-coloured, amber. Ears to be of good length, and carried close to the side of the head. The general conformation of the body must be that of a wellmuscled, compact, clean-knit dog, proportionate in all its parts. The breast to be of medium width, but deep; neck fairly long, but thick, with a good lofty carriage. Back of the loins broad. The nostrils in a white dog may be either black or liver-coloured, but in a black dog they must be black. Fore and hind limbs to be of medium length, with plenty of bone and substance, ending below in small, thick feet. The claws should be black, or dark, if the dog is chocolate or some colour other than black. The poodle makes an excellent companion and house-dog, but the uncertain nature of its temperament does not render it suitable for the companionship of children. It is a hardy variety of dog, but to keep it smart requires a good deal of attention, and some experience is necessary in order to clip it properly. The fashion of clipping changes. 


\section{The Dachshund.}

The dachshund in his native land is in reality a terrier, being used in Germany much as a terrier is used here. The transition from the terrier type of dog to one having certain hound characteristics has been brought about by breeders in this country, but the wisdom of this is open to doubt, because the dog is useful only as a companion, and lady exhibitors have always been the most numerous supporters of the breed. In appearance the dachshund closely resembles the beagle and Bassett hound, the most striking difference being in colour, most dachshunds being red, black and tan, chocolate and tan, fawn, or dappled, the lastnamed, spoken of as 'tiger dachs,' being somewhat scarcer than the others. The Kennel Club have a class allotted for dogs and bitches under $18 \mathrm{lbs}$. in weight, but these can hardly be regarded as a toy variety. The classes at the Kennel Club Show are always wonderfully well-filled. The body should be long and low-in fact, a dachshund can hardly be too long; the coat smooth, and perfectly flat, but there are three grades of coatthe smooth, the rough, and the wire-haired. If a smooth-coated dog, the hair must be fine, close, and short. The long-haired variety occasionally appears in a litter bred from the smooth. The 


\section{POINTS OF THE VARIOUS BREEDS 101}

head should be wedge-shaped, and the ears thin, long, set on high up, and covered with short hair. The nose is long, and the nostrils either black or flesh-coloured. The colour of the eyes in a darkcoloured dog are black, but in the red specimens the iris is brown. One of the most striking characteristics of the dachshund is in connection with the chest and fore limbs; the chest is remarkably deep, and the sternum or breast is so prominent that, when the dog is sitting on its haunches, the breast almost fills up the entire space between the fore limbs, and, even when the dog is at exercise, the sternum almost touches the ground. The fore-arms bend inwards, until the knees or pasterns are reached-in fact, touch each other at this point, and then bend directly outwards. This certainly constitutes a deformity, but the better the 'crook' the more typical the specimen. The toes must be long and flat, and the claws long, and in a black dog black, and in a brown or chocolate dog brown or dark-coloured, a white claw being distinctly defective. Another striking feature of these hounds is the looseness of the skin; no matter what part of the body you grip, you will find that the skin can be taken up in folds, and, unless there is a superabundance of loose skin, the dog will be of no use for the showbench. 
The hind limbs are longer than the fore limbs. Dew claws are frequently present, and it is advisable to remove these as soon after birth as possible, because they only become an annoyance to the dog. The tail to be carried similar to that of the fox hound, but never over the back. It has been said that the dachshund is of rather a quarrelsome disposition, but the writer's experience of the breed is that they are exceedingly docile dogs, good-tempered, and certainly not given to quarrelling, unless provoked. In Germany they are used a good deal for hunting the fox in his burrows, and in America they are frequently employed as a pack for hunting rabbits, but they are not in this respect equal to the beagle.

\section{The Chow-Chow.}

This is a very popular variety of the dog, the chief patrons of the breed being ladies, but why the fair sex should be so fond of these dogs the writer is at a loss to understand, there being many British breeds much handsomer than, and quite as companionable and good-tempered as, the chow-chow, which is a native of China. Ladies seem to be, however, particularly fond of importations, and usually give a hearty welcome to 'alien' doggies, in spite of the fact that many recent importations are of more than questionable 


\section{POINTS OF THE VARIOUS BREEDS 103}

descent, though all are eligible for competition under the vague classification of 'foreign' dogs. Chow-chows vary considerably in their colour, the most general colour being foxy-red, but black, yellow, white, etc., are not uncommon, and the Chow-Chow Club are favourable to any of these colours. The most important faults of the breed are a red tongue, a red nose, except in the light-coloured varieties, drop ears, and defective carriage of tail, that is, a tail that does not curl over the back, as it should in the chow-chow. Not only must the tongue be black, but the whole interior of the mouth. The coat must be abundant, coarse in texture, soft, and very woolly beneath; in fact, a dense undercoat is an important point, and, in brushing this variety of dog, the coat must be groomed in the direction of the head, so as to give a good stand-off appearance. The weight averages from 40 to $50 \mathrm{lbs}$, and the general conformation of the dog is good. The facial expression is keen, and like that of the Esquimaux dog; the skull is flat and broad, and muzzle, of medium length, has a blunt appearance. The ears should be set on well forwards, and carried erect. Back and loins strong, but not long, because these dogs are rather cobbily built; fore and hind limbs strong, straight, big-boned, ending below in round, catlike feet, with strong 
claws, well padded with hair between the toes. It must be mentioned that there is also a smooth variety of chows, but at a recent Kennel Club Show there were only three entries in this class, whereas there were thirty entries in the other classes.

\section{The Bull Terrier.}

This is a most useful variety of dog, and one that has always held its own in public estimation, for it is a difficult matter to find a better allround breed of terrier than the Bull. It makes a fairly good 'pal'; is hardy, usually of sound constitution, and clever at the destruction of smaller vermin; and as a guard for person or property has few equals, certainly no superior. There is a toy variety, the points of which are practically identical with those of the larger. The term 'Staffordshire bull terrier' is frequently used by 'doggy' men, owing, it is believed, to the notion that the bull terrier first of all sprang into existence in that district, as the result of crossing the bull-dog with a white English terrier. The old type of bull terrier had some sort of markings upon him, such as red, brindle, and so forth; in fact, in the advertisement columns of any of the 'doggy' papers there will usually be found for sale bull terriers with 


\section{POINTS OF THE VARIOUS BREEDS 105}

markings upon them. By careful selection the bull-dog element, such as the 'broken-up' face, markings, 'crank,' tail, etc., has been bred out, and the orthodox bull terrier is pure white, but markings on the toy variety which should be about $6 \mathrm{lbs}$. in weight, cannot be regarded as detrimental. The general appearance of a bull terrier should be that of a smart, active, heavybuilt, clean-limbed,deep-chested, powerfully-loined, strong-quartered, heavy-shouldered, intelligentlooking dog, absolutely free from throatiness, i.e. free from loose skin about the jowl, throat, or dewlap. The skull must be broad, the muzzle thick, the nostrils black, the teeth sound and level, and the jaws indicative of great power, so that the masseter muscles must be well developed. The eyes are rather small, and have a knowing twinkle in them; this is very characteristic of the bull terrier. A 'whip' tail, carried straight out, gracefully-sloping quarters, and powerful first and second thighs, are additional points of beauty. The ears ought to be small, and carried erect or semi-erect, the opening being directed forwards. A typical bull terrier should impress one as being, physically, a multum in parvo. These terriers are easily reared, and the prices for good specimens very reasonable. Cropping is no longer allowed in this country, 
but it is still practised in some others. In America there is the so-called 'Boston' terrier, which is practically identical with the bull terrier.

\section{The IManchester or Black-and-Tan Terrier.}

As the name implies, this variety of terrier originated in and around Manchester, and the chief patrons of the breed have been the working classes in Cottonopolis and other Lancashire towns. They are smart, active-looking terriers, with raven-black coats, mahogany-tan points, and black pencilling over the last-named in certain parts. Particular attention is paid to the correct marking, as so many black-and-tan terriers are defective in this respect. The coat ought to be smooth, short, moderately fine in texture, yet sufficiently dense to afford a good protection to the skin beneath. The head should be long, and finely chiselled; the ears and the eyes small; and the correct carriage of ear is close to the side of the head. Black-and-tan terriers, like White English terriers, often show too much of the bull-terrier element about the head, and a connoisseur would not look at a dog with this defect. The correct weight is from 16 to $18 \mathrm{lbs}$, certainly not above 20 lbs. ; for the 'toy' variety 


\section{POINTS OF THE VARIOUS BREEDS 107}

the average is from $3 \frac{1}{2}$ to 6 or $7 \mathrm{lbs}$. All limbs must be perfectly straight, of medium length, and the elbows placed close to the side of the chest. A 'whip' tail carried straight out, strong loins, neatly-turned quarters, and a graceful conformation of first and second thighs are necessary in a typical specimen. As previously stated, the tan markings are most important. They are distributed as follows :-

On the front of each knee there should be a round black mark; these are known as the 'thumb spots.'

On each cheek there should be a tan spot, termed the 'pea' marks.

On the lower part of the upper jaw there should be tan marking, and this ought to extend almost to the level of the eye. The lower jaw also should be tan-marked up to the throat.

On the inside of the ears there should be tan markings, also on the inside of the hind feet; the marking here sometimes extending up to the hocks, but on no account must the marking on the outside of the limb extend above the hock. If it does so, the dog is said to be 'breeched,' and that is one of the most serious faults a blackand-tan terrier can have. Both fore-feet must be tan-marked about half-way up, with pencil or black markings running up each toe. The 
tan marking beneath the tail should not be discernible when the tail is at repose.

These terriers are hardy, make good companions, and many of them are very clever at killing rats, etc.

\section{The Fox Terrier.}

Of all the varieties of terriers none has ever been held in greater esteem than the Fox terrier, which is spoken of as being 'smooth-haired' and 'wire-haired,' the latter having within the last few years somewhat displaced the former. The even temperament, medium size, and working qualities have rendered the fox terrier a companion alike for rich and poor. The correct colour for a fox terrier is white, with or without markings, blackand-tan markings being very common upon the head and ears, with or without one or more patches upon the body. The head must be long, and the skull flat, so that when viewed in profile there should be little or no evidence of stop. Any tendency towards shortness of head is distinctly defective, and judges are very particular about quality in this region. Idstone said that a fox terrier should be able to lick the bottom of a pint mug, which necessarily implies the possession of a long head. Eyes small, beaming with intelligence, dark in colour, and expressive 



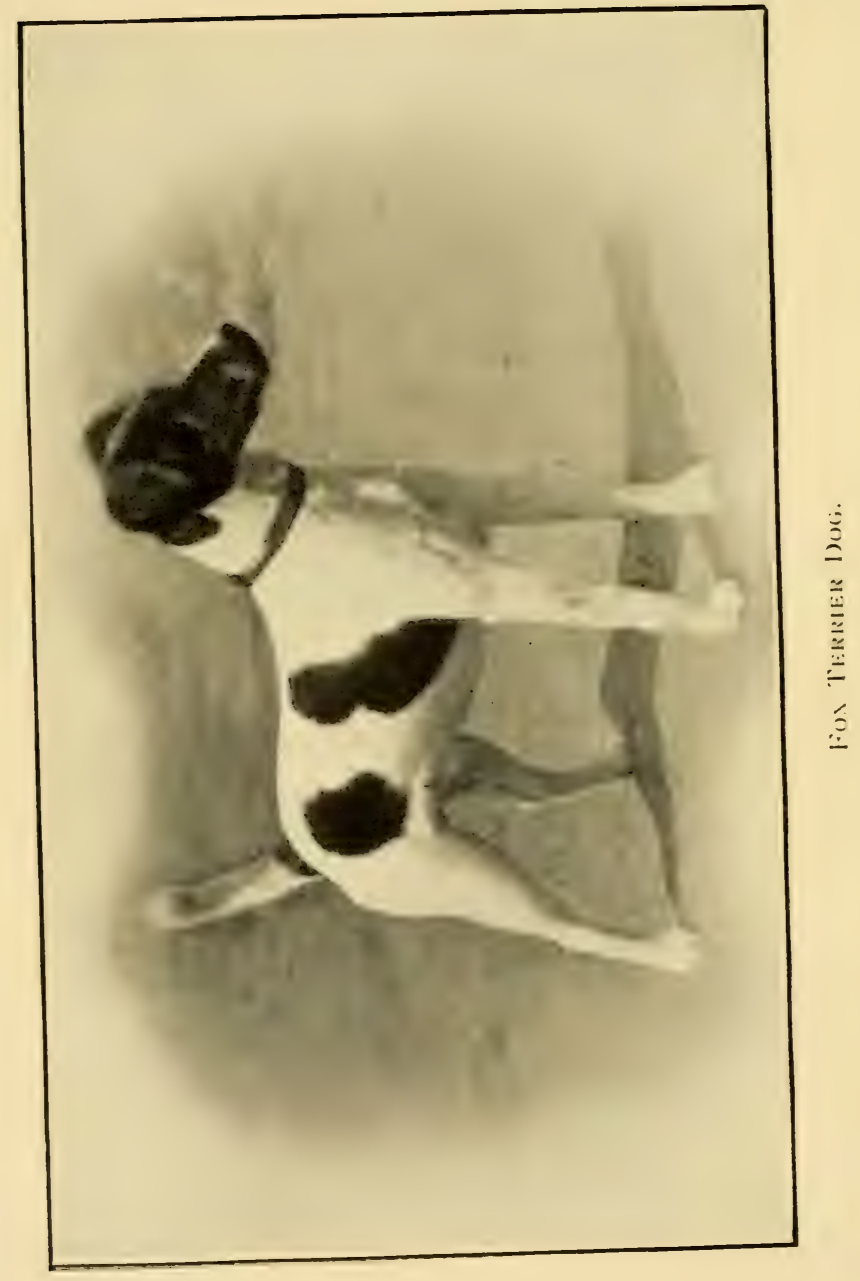




\section{POINTS OF THE VARIOUS BREEDS 109}

of keenness in every sense of the word. The correct type of ear is the so-called V-shaped one, carried forwards so as to show the face of the V. There must not be much falling away below the eyes, and the outlines of the jaws should be clean, i.e. free from any underline of cheek, etc. The nostrils must be black; the teeth sound and level, and, if a wire-haired terrier, there should be a moustache on either side of stiff wire hair. The hair upon the ears must be short, and fine in texture. Neck of medium length, obliquely carried, passing into gracefully-sloping, fine shoulders. Judges are very keen on quality in the regions of the shoulders, as many fox terriers become much too coarse. The fore limbs of medium length, straight as arrows, ending below in round compact feet. Passing on to the chest and withers, the former must be deep, and the withers gracefully sloping on to the short back and strong loins. 'The tail must be 'docked,' and carried gaily. The hind-quarters must show as much quality as the fore, and every one purchasing a terrier should not only view it from the front and side, but also obliquely, and directly from behind. In this way one can ascertain whether a dog is well-coupled fore and aft, and judge as to the conformation of the fore and hind limbs. The first and second thighs ought 
to be well clothed with muscle, the hocks of neat shape, and the pasterns and toes well formed. In the smooth rariety the coat must be close, of medium texture, dense, and the belly and underside of the thighs must be clothed with hair. In a wire-haired fox terrier, the hair must be of a bristly nature; in fact, it cannot be too hard, but it must be smooth and dense. A thin, open, or soft coat is about the worst fault one of these terrier's can possess. A cherry nose, tulip or rose ears, and a mouth under- or over-shot, are disqualifying features. The general build of a fox terrier must correspond with that of a wellmade cob, which implies good conformation from nose to feet.

\section{Scottish and West Highland White Terriers.}

Scottish Terriers are sometimes spoken of under the appropriate title of 'die-hards,' also as Cairn Terriers, owing to the nature of the work to which they have been trained. In Skye they are often spoken of as 'otter' dogs; the term 'Aberdeen' is in general use, and it is synonymous with 'Scottish,' but the popular idea is that the two terriers are distinct. The White West Highland terrier, sometimes spoken of as a 'Poltalloch' or 'Roseneath' terrier, is in reality a variety of the Scottish, but, now that a 


\section{POINTS OF THE VARIOUS BREEDS 111}

distinct club has been formed, the White West Highland terrier, or more correctly the West White Highland terrier, is sure to become a popular variety of dog. Both the Scottish and the West Highland are well worthy of the praise that has been bestowed upon them, more especially by their Scottish admirers. Both exhibit a remarkable degree of intelligence, when properly trained; are extremely hardy; game to death, fighting with all the dash and courage characteristic of the true Highlander; and well merit the name 'die-hard.' Any one in search of a firstclass companion, either for town or country, cannot do better than purchase a specimen of the Scottish or White West Highland terrier. The typical colour for a Scottish terrier is brindle or black, but sandy and fawn specimens are common, but not so much thought of as the former. The presence of white hair is detrimental to either. The general conformation must be that of a long-bodied, short, hard-haired, erecteared, sturdily-built terrier, weighing from 16 to 20 lbs., but many show specimens are heavier. The harder the coat is, the better, and it must also be close and dense. The ears large, erect, and pointed; skull broad; muzzle broad and strong; in short, the Scottish terrier has a heavy facial expression, with keen, dark-looking eyes. 
The general points of the White West Highland terrier are similar to those of the Scottish.

\section{The English White Terrier.}

In general appearance and build the English white terrier is closely allied to the Manchester or black-and-tan terrier, though it is not as popular as the variety last-named. Both breeds have, within these last few years, gradually declined in popularity, the older fanciers having fallen out of the ranks, in the belief that these terriers have lost their 'smart' appearance since the Kennel Club put their veto upon the practice of cropping. Another reason why the white English terrier is not popular is the difficulty so often experienced in breeding true to type. In weight a white English terrier should be about 15 or 16 lbs., this being the most useful size, but there is also the 'toy' variety, weighing 6 or 7 lbs., the last-named being useful only as lapdogs. They are very smart-looking dogs, and the body and limbs must be spotlessly white, any kind of marking being exceeding detrimental. The head should be long, ears small and carried close to the side of the cheek, eyes of the medium size, dark, and full of intelligent expression. The nose black, the chest deep, the fore-limbs perfectly straight, the hind-quarters 


\section{POINTS OF THE VARIOUS BREEDS 113}

of a graceful slope, and the stifles neatly turned. The tail must be of the so-called 'whip-like' character, carried straight out; in fact, judges are very keen upon quality in the hind-quarters, any tendency towards the bull terrier element being decidedly faulty. For companionship the writer cannot recommend these dogs, owing to a combination of reasons, into which it is not necessary to enter.

\section{Airedale and Welsh Terriers.}

THE AIREDALE TERRIER.

This is a capital variety of terrier, and appears to have had its origin round about Bingley, Saltaire, and the surrounding localities in Yorkshire. Previous to the adoption of their present title they were known as 'waterside terriers,' chiefly because they were used for such purposes as ratting on the river-banks, etc. It is a brokenhaired variety of terrier, of a very hardy nature, and one that makes an excellent companion, and, if properly trained, a serviceable gun-dog or watch-dog. The correct colour for an Airedale is grizzle and tan, and the average weight ranges from 35 to 40 lbs., but an Airedale must be neither under-nor over-sized. The chief points are a hard and grizzled coat, a long flat skull without any evidence of stop, small V-shaped ears, sound teeth, 
straight big-boned fore and hind limbs, and a black nose, a flesh-coloured one being a disqualifying feature. The tail should be docked as soon as the puppy is a few days old.

\section{THE WELSH TERRIER.}

As the name implies, this variety of terrier is regarded as being indigenous to Wales. It is in general conformation very like, and is said by Welshmen and other supporters of the breed to be quite as good a worker as, the fox terrier. The correct colour for a Welsh terrier is grizzle and tan. Like the Airedale terrier, the puppies should be docked when a few days old. The skull ought to be flat, with little evidence of stop between it and the nose, the nostrils of which should be black. Ears small, V-shaped, and carried close to the side of the head. Neck of medium length, ending in fine shoulders, deep chest, straight fore-arms, short pasterns, and close feet; carriage of the stern like that of the fox terrier. The coat should be hard in texture, close, of medium length and blue colour, with tan points on the face and limbs. Welsh terriers are not difficult to rear, but the variety is not in particular demand, although there is usually a fair number of entries at the Kennel Club Shows, and at the principal Welsh Shows. The 



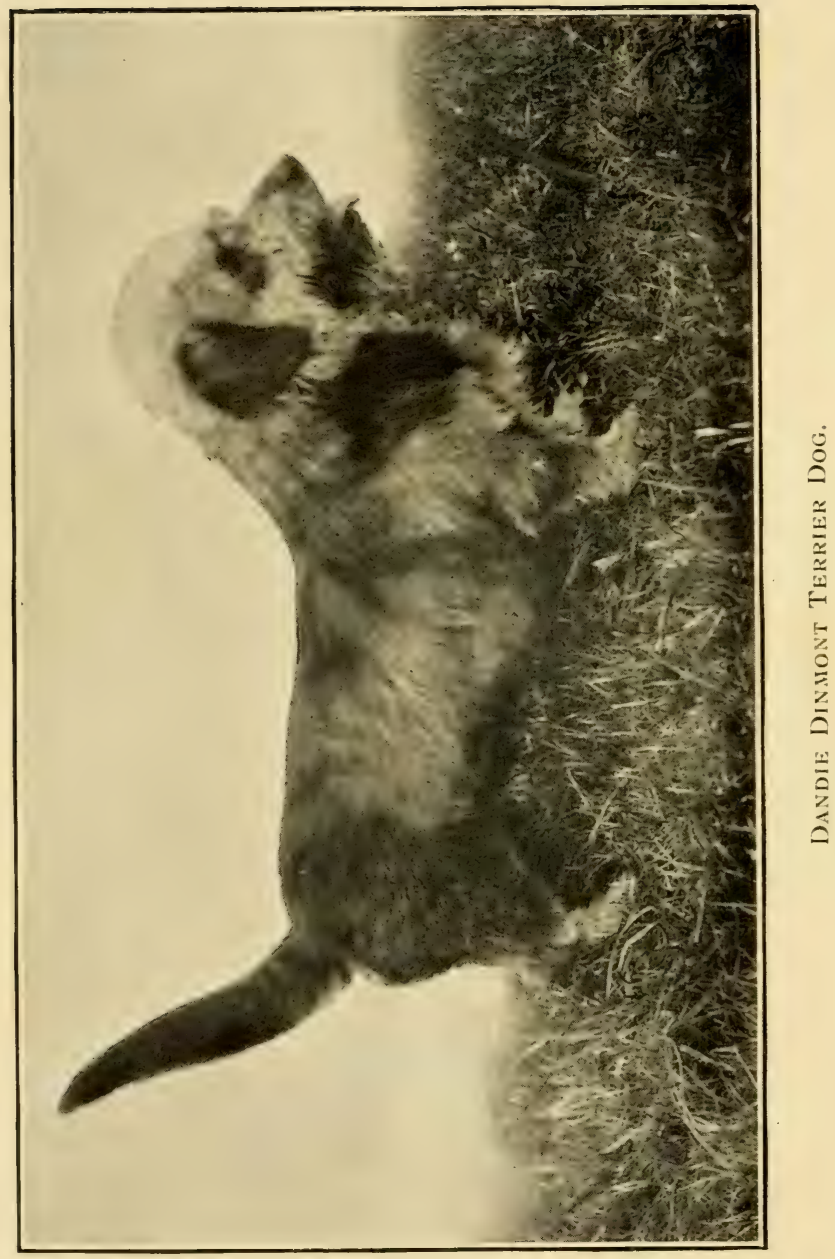




\section{POINTS OF THE VARIOUS BREEDS 115}

temperament of these terriers is usually excellent, and they make capital companions, house dogs, etc.

\section{Dandie Dinmonts and Bedlington Terriers.}

\section{THE DANDIE DINMONT.}

This is a capital little terrier much the same size as the Skye terrier, similar in height and length of body, but stouter in build. There are two varieties, one known as the 'pepper,' the other as the 'mustard,' in accordance with the shade of hair. The Dandie is particularly suitable for what may be described as 'working in tight corners,' his short legs and long body enabling him to hunt where another dog, such as a fox terrier, would be unable to go. The Kennel Club's classification is for dogs any colour, pepper dogs, and mustard dogs, and these classes are usually very well filled. The coat of a Dandie should be about two inches long, and of medium texture, the hard and soft hair blending. The coat is known as pily or pencilled. The hair extending from the head to the set on of the stern ought to be a mixture of hard and soft, and in colour that on the under part of the body is lighter and softer than that on the back. In a pepper-coloured dandie the hair is either dark, bluish-black, or a light silvery grey; the mustards vary from a reddish brown to a pale fawn, but 
the hair on the head is of a cream colour, whilst that on the legs and feet is darker. The eyes should be full and round, deep hazel in colour; the ears large, carried close to the side of the head, and have a thin tip of feathering around the margin. The hair upon the ears should be soft and brownish. The length of the ears is about four inches; head large, skull well-domed, and broad between the ears. From the muzzle the hair should be darker; strong sound teeth and powerful jaws are a sine qua non. Neck strong, back of the loins strong; the fore-limbs straight, very muscular, covered by tan hair in a blue dog, but in a mustard specimen darker in shade. All Dandies are a trifle higher behind than in front. Height from 9 to 11 inches, and weight 14 to 24 lbs., but breeders prefer dogs weighing about 18 lbs. The Dandie Dinmont is extremely hardy, and makes an excellent companion.

\section{The Bedlington Terrier.}

This variety of dog is said to have originated at Bedlington in Northumberland, having been bred there for the purpose of waterside work, etc. Pedigrees of these dogs can be traced back as far as 1792, but Thomas Ainsley, in 1825, seems to have had considerable influence in moulding the type. Bedlingtons are exceedingly game 
terriers weighing about 24 lbs, with a hard coat that does not lie flat to the sides, and either sandy, sandy and tan, dark blue, blue and tan, liver or liver and tan in colour. The head is very distinctive. The outline of the dog reminds the writer somewhat of the Irish water spaniel, but some persons may not be able to see the likeness. The head, which is distinctive, is narrow, and the skull domed, with a well-marked tuft or top-knot. The ears are large, thin, covered with silky hair, and of the so-called filbert shape. Neck long, and deep as it approaches the shoulders; fore and hind limbs straight; back and the loins rather long, with a straight tail, about 9 inches in length, feathered along its lower border, and coming to a fine point. The general build may be described as 'lathy,' nevertheless the dog is one that can hold its own in a 'turn-up' with any other dog of its own weight; in fact, the Bedlington is the gamest of the game. In order to keep the dog in good condition, great attention must be paid to the coat, etc.

\section{Irish Terriers.}

Irish terriers, more popularly known under the title of 'dare-devils,' are an exceedingly popular variety of terrier, and the breed being particularly 
suited for residence abroad, large numbers are constantly being exported from this country to Africa, India, etc., where high prices are frequently paid by fanciers for typical specimens. The colour of the Irish terrier is red, and its general build similar to that of the fox terrier, but a little larger. The coat, an important feature, must be perfectly flat, and as hard as pin wire. A coat that is inclined to be loose, curly, or shaggy is a faulty one. The average weight may be taken as ranging between 16 and 25 lbs., but a nice size is 22 lbs. As previously stated, red is the principal colour, but wheaten yellow and grey colours are frequently met with. The skull should be long and flat, narrow between the ears, without any sign of stop. Below the eyes there should be a slight falling away, whilst the jaws should be strong, and bear sound level teeth. The hair on the head must be short and straight, excepting the beard upon the muzzle, which gives the Irish terrier a very characteristic appearance. There should be no loose skin about the jaws, throat, etc., and the body must be compact throughout, the chest deep, the back and loins strong, and the tail of a gay carriage. The presence of white hair on the feet, a 'Dudley' nose, i.e. a fleshcoloured nose, an under- or over-shot mouth, and a brindle colour, are disqualifying features. 



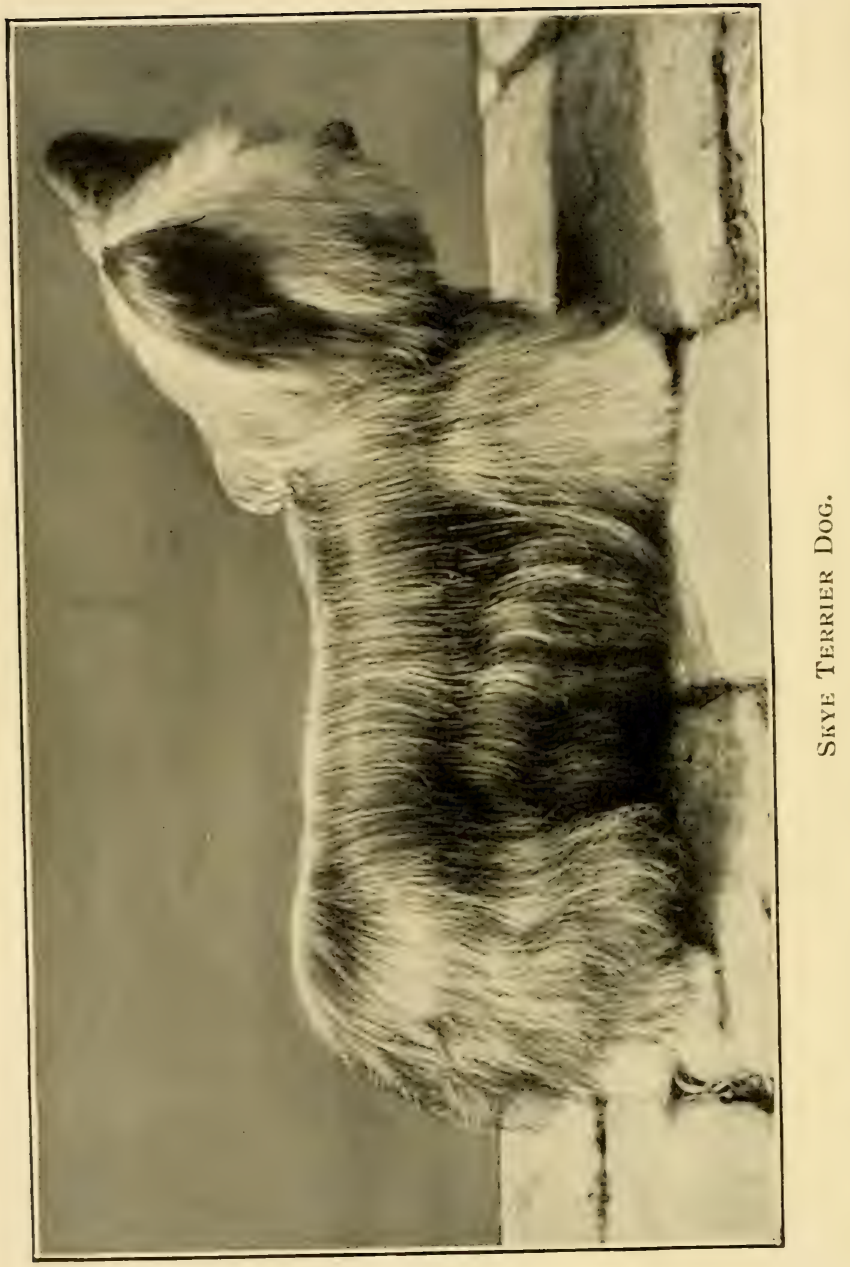




\section{Skye Terriers and Clydesdale Terriers.}

Both the Skye and the Clydesdale are exceedingly useful varieties of terriers, but the former is held in much greater esteem than the latter; in fact, the number of Clydesdale terrier breeders is comparatively small. There is no particular reason for the preference of the one over the other, for the difference between the two variations is not great. The Skye terriers are divided into 'dropears' and 'prick-ears'; in the former the ears lie close to the cheeks, whereas in the latter the ears are erect, with a slight fringe hanging over at the top of them. Both the Skye and the Clydesdale terrier are long-coated, long-bodied, short-legged varieties of dog, originally bred for the purpose of destroying vermin, etc., but the long coats now seen in typical specimens hinder work, and it is preferable to have a medium length of coat. The Skye terrier, as the name implies, is thought to have originated in the island of that name, and the Clydesdale in the Vale of Clyde. The latter is to the Skye terrier what the West White Highland terrier is to the Scottish. The typical colour for a Skye is steel blue, but other shades are fawn, and black and slate. If steel-blue, the coat should be tipped with black; if fawn, with black or dark brown. The weight varies from 
about 16 to $20 \mathrm{lbs}$, and the height is about 10 inches at the shoulder, with a length of body nearly 40 inches. The outer coat ought to be composed of hard, long hair, with a woolly undercoat; the coat must be perfectly straight, and it is usual to have it parted down the centre from head to tail. The hair on the head overhangs the eyes, partly concealing them. The undercoat is thick on the body, but absent on the head and legs. The tail should be carried low with a dropear dog, but on a level with the back in the prick-ear variety. Eyes dark brown or hazel, medium-sized. To keep a Skye terrier in good condition, the coat requires a good deal of grooming, hence it is necessary to make free use of the comb every day. The coat of the Clydesdale terrier is softer than that of the Skye, its texture being of a silky nature. The back is often blue, with a silver shade on the legs, but the commonest colours are the different shades of fawn. The coat must never be curly; ears must be small, erect, placed high on the top of the skull, and have a fringe of hair upon their tops and sides, blending with the whiskers and moustache. The Kennel Club attach so little importance to Clydesdale terriers that there is no class for them at the shows.

Both varieties of terriers make excellent com- 
panions, they are hardy, but of variable temperament, and the writer considers them not as useful, so far as companionship, etc., goes, as the Dandie Dinmont.

\section{Bull-Dogs and Toy Bull-Dogs.}

\section{THE BULL-DOG.}

The extreme popularity of the bull-dog, both in the ordinary size and toy varieties, renders it necessary that a somewhat extended account of the breed should be given, but the writer will not attempt to move the mists that enshroud the origin and early history of the dog. The present British bull-dog has been built up out of the poorlooking specimens depicted in prints of only eighty years ago. In a book on the bull-dog by H. St. John Cooper, there is reproduced a celebrated bull-dog called 'Little Billy'; this illustration depicts about as miserable a specimen of a bulldog as mortal man could conceive. The anatomical outlines of the bull-dog are present, but that is the most that can be said. At this period the dogs were used for bull- and bear-baiting. There is a Spanish bull-dog, and many believe that the bull-dog of to-day has been derived from the Spanish bull-dog, as the pointer was from the Spanish pointer. Many bull-dog breeders consider that there is a good deal of mastiff in the animal, 
this cross having been introduced for the purpose of giving the necessary courage, but the modern bull-dog in this respect bears no resemblance to his ancestors. The colour of the dog is not important; in fact, these dogs exist in a variety of colours, the chief being brindle, white, red, fawn, brindle and white, fawn and white, light or dark brindle. A black, slate, or dark-brown bull-dog does not find favour. As regards weight, $45 \mathrm{lbs}$. may be taken as the average, but this varies. The toy varieties also vary, some being about 18 lbs., but any dog or bitch that exceeds 20 lbs. is disqualified for show purposes. A typical bull-dog must have a large skull, with a flat forehead, broad temples, and above the nose a deep depression called the stop. The nose to be jet black, and the face short, and well wrinkled. The chops or cheeks must be thick, the flews hanging well down below the lower jaw, with a superabundance of skin about the throat and neck. The lower jaw powerful and square, projecting beyond the upper one in an upward direction. This is sometimes spoken of as the 'turn-up.' The teeth are strong. The typical form of ear is that known as 'rose,' which should be set high on the head, thin in texture, and covered with fine hair. The terms 'button,' 'bat,' and 'tulip' ears are often given to other shapes of ear, and bull-dog breeders 
regard these forms as very defective. Eyes dark in colour, round, and placed low on the face. Chest very wide, deep, and shoulders powerful and muscular to a degree. Body strong, short, with great width across the shoulders, but narrower as the loins are approached; these must be arched, constituting the so-called 'roach' or wheel back. Fore-limbs strong and straight, with powerfully developed muscles running down the fore-arms. It is the great degree of muscular development in this region that produces the bent appearance, but this is only apparent, and any bull-dog with bent fore-limbs is very defective. The feet well arched, and the toes turned outwards. The chief defects of a bull-dog are: tulip, button, or bat ears, the button ear being one that falls over and forwards, thus hiding the passage of the ear; a 'Dudley' or flesh-coloured nose, which is a disqualifying feature; pinched nostrils, in which the nose is small and attenuated; terrier eyes, indicated by the white of the eye being shown when the dog looks from the front. A round skull is objectionable, as the skull should be flat and broad. From 17 to 20 inches may be taken as the average measurement of a bull-dog's skull, the measurement being taken between the ears and eyes, or over the temples. Many bull-dogs are spoken of as being 'frog-faced,' owing to the 
peculiar appearance some of them have. It is due to a deficiency of under-jaw, in other words the dog is overshot: this is a serious defect. Other defects are deficiency in bone, a thick coarse tail, flat sides, splay feet, legginess, and a want of true bull-dog expression throughout. The tail should be set on low, straight, thick at the root, about six inches in length, covered by fine hair, and carried straight downwards. Many believe that a 'screw' tail, others that a 'crank' tail, i.e. one with kink in it, is the correct type, but the description given is the one recognised by the British Bull-Dog Club. A toy bull-dog should have a wide chest, short back, short tail, and straight fore and hind limbs, but the hind quarters must be lighter than the fore, ears either rose or tulip, the eyes of moderate size; in fact, the points of the toy bull-dog are identical with those of its prototype. As previously stated, the weight must be under $20 \mathrm{lbs}$.

\section{The Yorkshire Terrier.}

At one time the Yorkshire terrier was exceedingly popular, more especially amongst fanciers in his native county, but the majority of lady fanciers prefer a lap-dog with a coat that requires less attention than does the Yorkshire's. This dog is a long-haired, silky-coated, toy terrier, 


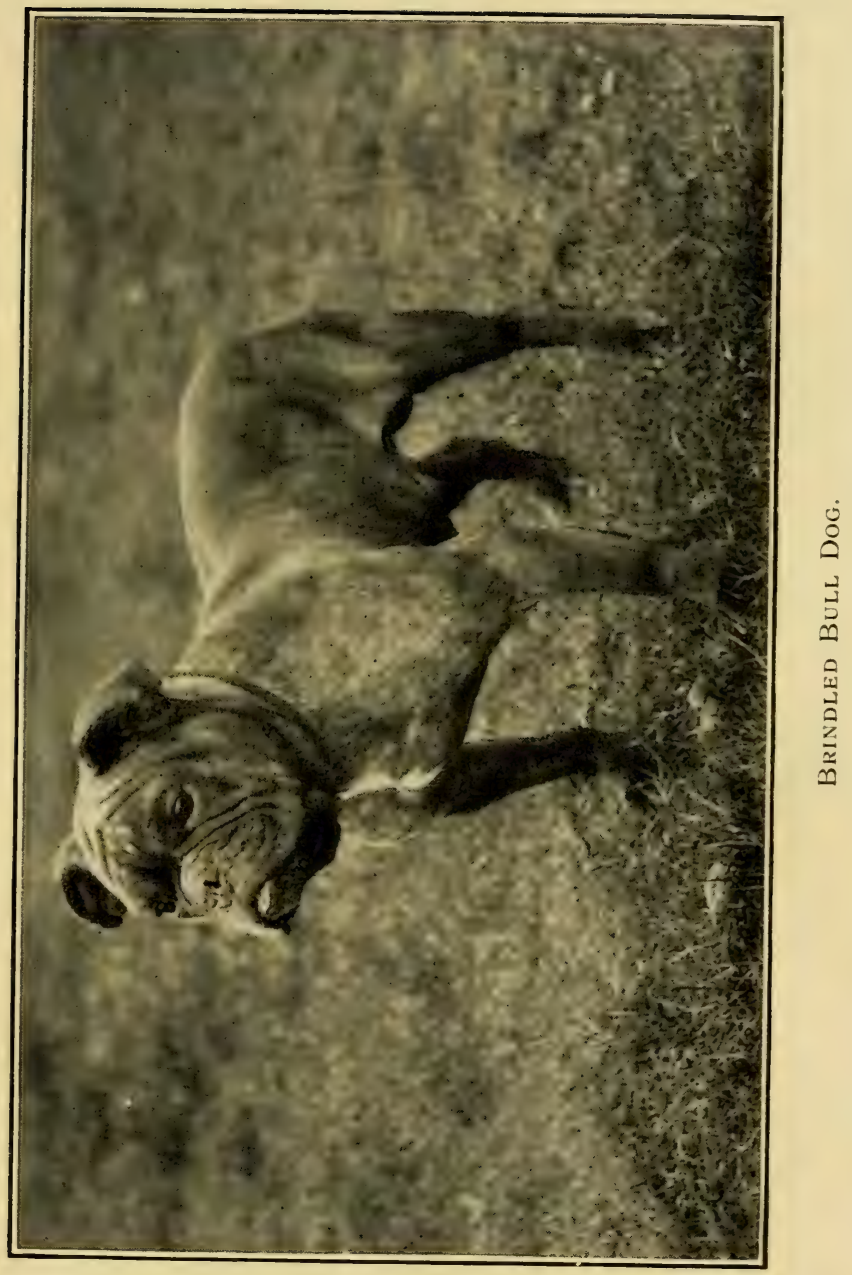



varying in weight from 4 to 7 lbs., but the smaller weights are usually the most sought after. The coat and the colour are very important points. The former must be long, the longer the better, and of a silky texture; what are called the 'whiskers' ought to hang down to the ground. The hair on the head and back is parted, and of a silver or steel-blue colour. Tan-markings are present over the head and face, and on the feet, and should be well defined, and of a nice rich colour. The hind-legs ought to have tan-coloured hair upon them; the head should be small and narrow, the ears small and fine, the body compact, and the general expression vivacious. Although the Yorkshire is a lively little terrier, and as a rule of good temperament, it is distinctly inferior to other breeds as a companion for children. In order to get these terriers ready for exhibition a good deal of care is necessary, and an owner, unless he is prepared to devote much attention to the toilet, had better not take up the breed for show purposes. The Yorkshire terrier must not be washed too often, otherwise the texture of the coat is destroyed. It is, of course, necessary to wash the animal occasionally, but reliance should be placed chiefly upon the brush and comb. 


\section{The Schipperke.}

The Schipperke is a short-haired black dog, occasionally chocolate, and it is said that white specimens occur. The breed originally came from Holland and Belgium, being used by bargemen, and they get their name through having been the constant companions of skippers. There are a good many of them in this country, and the breed has been vastly improved since its introduction. The coat must be perfectly black, the presence of white hair being objectionable. The weight most preferred is about $12 \mathrm{lbs}$. The coat is smooth, except upon the neck, the hair of which should form a frill, whilst that at the back of the thighs must be profuse; this being known to fanciers as the 'culotte.' The head should be broad in the skull; the eyes a medium size, and dark brown; the ears erect, giving these little dogs their very sharp expression; the back short and straight; the neck short and well-arched; the forelimbs perfectly straight, of medium length; and the hind-quarters well rounded. The Schipperke has no tail, or, if there is one at birth, it must be immediately cut off as close as possible to the body. The chief faults are drop ears, light-coloured eyes, a light nose, a projecting lower jaw, and light claws. The last-named ought to be perfectly 


\section{POINTS OF THE VARIOUS BREEDS 127}

black. The Schipperke is of hardy constitution, and puppies are not difficult to rear, but these dogs are useful only as companions.

\section{The Italian Greyhound.}

This variety of toy-dog is built upon much the same lines as its prototype, the greyhound, but the little creature is only a lap-dog, and not of much use. At the Kennel Club Show there are usually about a dozen entries, and the breed never makes particular headway as regards popularity, although there is a club watching its interests. The reason of this is, in all probability, the extremely delicate nature of the dogs. The weight should be from 3 to 10 lbs., but the smaller they are the better. Regarding the colour, there is considerable choice, but fawn, either light or dark, is the commonest, and black the rarest. A not uncommon colour is blue or slate; judges do not, however, attach a great deal of importance to this matter; the skin must be fine, and the coat perfectly flat, and of soft texture. The arms and fore-arms are long, the pasterns long, clean, and fine at the joints. A deep chest but certainly not a wide one, a long neck, a long back, powerfully-arched loins, long first and second thighs, and a long stifle are points of the Italian greyhound. Look- 
ing at an Italian greyhound, one should at once see that it has a well-developed muscular system, the individual muscles or groups of muscles being plainly marked in outline, exactly as in the larger breed. Judges pay a good deal of attention to the head and the correct carriage of the ears, so many of these little dogs being faulty in this region. The head ought to be long, beautifully chiselled, and have small semierect thin ears, set-on well back. Nose to be black. The head, if it has any tendency towards roundness, constituting the so-called 'apple head,' is faulty. The same remark applies to 'tulip' or erect ears, to a broad chest, and to a bad conformation of the feet. Italian greyhounds are, almost always, sweet-tempered little dogs, and good, though not the best, companions for children.

\section{The Pomeranian.}

As a lap-dog the Pomeranian is more extensively patronised than any other variety. It was originally imported from Pomerania, in Germany, but it is only since its introduction into this country that the breed has attained its present high standard of excellence. At one time the Porneranian or Spitz dog was common enough in this country as a small white dog weighing 


\section{POINTS OF THE VARIOUS BREEDS 129}

about $20 \mathrm{lbs}$, whereas in the present day some of them do not weigh above $3 \frac{1}{2}$ lbs. The Kennel Club's classification is as follows:-White dogs, any weight; black dogs, any weight not exceeding 8 lbs.; brown dogs, any weight; shaded sable, any weight; orange, any weight; blue, any weight; any colour and any weight, excepting white, black, orange, and brown. This classification shows how many varieties there are. Breeders usually prefix the word 'toy' when the little creature weighs less than about 6 lbs. The principal colours are black, white, chocolate, fawn, blue, and red; there are also parti-coloured dogs. Some breeders say that the red Pomeranians are the most troublesome to rear, but it is difficult to understand why this should be so. It is preferable to try to keep to whole colours, because breeding from parti-coloured dogs is apt to lead to the introduction of defective colouring of coat in whole-coloured specimens, such as, for instance, a patch of white hair upon some part of the body. The presence of white hair upon the breast is exceedingly common, and breeders are usually willing to sell specimens with this defect at a greatly reduced price. Any one purchasing a Pomeranian ought to scrutinise the breast carefully, because a tricky vendor will often dye the white hair to match the rest of 
the coat, or, what is still worse, will take a pair of scissors and snip them out, or else pluck them. The head of a Pomeranian is small, with a foxy expression about it, the erect ears adding to this appearance. The Pomeranian Club says that the head should be wedge-shaped, with a trifling evidence of stop in profile, whilst the hair covering the head must be smooth and short; the teeth level; the eyes dark and oblique. The same Club points out that the eyes should be expressive of great intelligence and docility. With all due respect to the formulators of this code of points, the writer would like to say that this last statement is hardly correct; in fact, for peevishness and a generally disagreeable nature he considers the Pomeranian is hard to beat. Doubtless admirers of the breed, who are mostly women, will say that the want of intelligence rests with the author and not with the dog; nevertheless the conclusion has been arrived at from practical experience of the Pomeranian, both in health and disease.

Returning to the description, the nose should be black in black-and-tan, black, or white dogs, but in all liver-coloured Poms, flesh-coloured. A short neck, covered with a profuse mane of long, straight, glossy hair, extending from the under-jaw and covering the withers, shoulders, 
and chest, is a point of great importance. The body short and compact; legs of proportionate length, ending in compact, small feet. The tail must be twisted tightly over the back, or else rest flat either on the right or left side of the back, and should bear long flowing hair. The undercoat should be soft and abundant, the outer straight and endowed with a high degree of lustre. A whole-coloured dog, if it has white hair on the feet, will be debarred from competition in a class for whole colours. The prices for Pomeranians vary considerably, but a really excellent puppy can be obtained for three or four guineas. The eyes of the Pomeranian often cause the owner some anxiety, owing to their weeping or watery condition. This is partly natural, but if the dog is allowed to sleep with its face exposed to a draught, a chronic inflammation of the eyes is liable to be set up.

\section{The Pug.}

There are two varieties of pugs, the black and the fawn, both of which have always been very popular, but during the last ten or twelve years the black variety seems to have had the largest number of patrons. A rough-coated variety of pug has been introduced, but never encouraged; and the term 'toy pug' is applied to the smaller 
varieties of this breed, but has no significance amongst pug-dog breeders. Light and dark shades of fawn exist, also apricot and silver fawns. When black pugs were first of all introduced, a great difficulty was experienced in breeding in the compact form that is so characteristic of the pug. Another difficulty was in connection with the coat, many black pugs having a rusty tinge. These defects have, to a large extent, been eradicated, and the black pug now breeds fairly true to type. Both the black and fawn varieties have occasionally a patch of white upon either the breast or the feet, and a patch in the latter place spoils the dog for a show. A typical pug should have a black mask, a black trace extending from the head to the set-on of tail, and black shading from the ears and feet. These markings should always be sharply defined, though many pugs, even prizewinners, lack this clearness of definition. The skin over the body ought to be very loose, and heavily-wrinkled, more especially in the region of the neck, shoulders, and back. The mask should be of intense blackness, and the nostrils black. The less the nose shows the better; the stop, that is, the depression in the face, should be deep, and the face ought to be well wrinkled. The head large and round; the ears small and thin, carried 


\section{POINTS OF THE VARIOUS BREEDS 133}

close to the side of the head, and covered by soft hair. The so-called 'rose' or 'button' ears are preferred by connoisseurs. Judges are very keen on quality in the region of the head, and like a pug that has a short, square, blunt muzzle. The chest ought to be broad and deep; the neck short and thick; the ribs well sprung, but the body short; the fore-limbs of medium length, well-rounded, and straight, with black toe-nails, though many fawn-pugs win that are lacking in the latter qualification. The tail should be doubly-curled, the tighter the curl the better. Large, dark, round, full eyes, moles on the cheek, and a thumb-mark on the forehead, are additional points of beauty. The chief defects are badlydefined markings, a want of general compactness, cow hocks, bad carriage of tail, white hair on the feet, deficiency of stop, protruding tongue, legginess; being too big, or too long in the head, or undershot; and, in black pugs, the appearance of bronze or fawn shading in the coat. Any one selecting a pug-puppy should endeavour to obtain one built on the lines of a cob, which implies that it must have a good pedigree, though all pedigree dogs are not necessarily of good conformation. The extremely docile temperament of the pug makes it the very best companion for children. A six weeks old puppy, of fashionable 
breeding, can be purchased at two or three guineas, and an older dog, say from six to twelve months, at three or four guineas. Pugs are of a fairly hardy constitution, and puppies not particularly difficult to rear.

\section{The Griffon Bruxellois.}

These little dogs originally came from Belgium, and they have become very popular in this country. They are quaint terriers, and their diminutive size makes them good lap-dogs. They are believed to be a mixed variety, chiefly consisting of toy bull-dogs, pug, stable griffon, and toy spaniel; the smaller they are the better, the weight most favoured being about $5 \mathrm{lbs}$. In colour they are either red or sandy, the coat being long and thick, and of a harsh or wiry texture. The lower jaw projects ; in fact, for this to be slightly undershot is characteristic. The eyes are black and large, with overhanging eyebrows, and the ears semi-erect, but neatly carried. The head must be large and round, and bristlelike hairs should be on the top, and a moustache should grow from the side of the nose and cheeks. It is this moustache that gives these little dogs their quaint facile expression. The body must be neatly-formed, and the limbs straight, ending in neatly-shaped feet, which 


\section{POINTS OF THE VARIOUS BREEDS 135}

must not be long. The tail should be carried in an upward direction. A typical Brussels Griffon generally commands a good price, and there is always a market for first-class puppies.

\section{The Maltese Terrier.}

This is a very ancient variety of terrier, and one that is extremely handsome, though it has never been particularly popular, doubtless because of the large amount of care that it is necessary to bestow upon it in order to keep it in first-rate trim. A typical specimen should be pure white, with a long and abundant coat, of an extremely silky texture. The Maltese terrier is regarded as being one of the oldest varieties of dog known, there being records of its history for several thousand year's. The whole of the body should be clothed with, and the face completely concealed by, fine silky hair. The nostrils must be of an intense black, a Dudley or flesh-coloured nose being a serious detriment. Breeders of the Maltese like to obtain specimens as small as possible; in fact, the smaller animals are the more highly prized. From 5 to $7 \mathrm{lbs}$. is the average weight. These terriers are fairly hardy, but not always of the most agreeable temperament. Excellent puppies can, as a rule, be bought at prices ranging from two to five or six guineas. 


\section{The Japanese Spaniel.}

These toy-dogs are not quite as popular as the Pekinese, but are built very much upon the same lines. They are sometimes spoken of as 'sleeve' dogs, and are natives of Japan. In all probability it is a very ancient variety, possibly having existed as long as the Maltese terrier. They are of fairly hardy constitution, but the prices which they command are certainly not as high as the Pekinese. The Kennel Club's classification is for dogs under, and dogs over, 7 lbs., but it is the Japanese Club that has done so much for the Japanese spaniel. The colours most favoured are red and white, and black and white, more especially the last-named. The markings must, however, be sharply defined, and uniformly distributed over the cheeks, ears, and body, irregularity of marking being detrimental. The weight may be put down as ranging from 5 to $10 \mathrm{lbs}$., but these, as in almost every other variety of toy dog, are preferred as small as possible; in fact, one of the aims of lady-breeders seems to be the reduction of size, without considering the future welfare of the breed. As in the Pekinese, the chest must be wide and square, back short, the legs and feet well-feathered, the coat straight, silky, and abundant, absolutely free from any 
tendency to waviness or curl, but standing 'well off'; and the more feather there is on the limbs the better. The skull should be broad, and the muzzle wide and strong; the eyes dark, and the ears $V$-shaped and well feathered. The tail should curl tightly over the back, and have a good plume. Extending from the nose over the front of the skull, there should be a white Vshaped area. The nose must be turned well up, and the dog active, and intelligent in facial expression. Any one contemplating going in for this variety of dog should buy a good bitch-pup, say at ten guineas, and mate her with a suitable sire. The dog-pups, though preferable for companionship, are lower in price.

\section{The Pekinese.}

These toy-dogs originally came from Pekin, and during the late disturbances at that place several specimens were brought over to this country, but most of the show dogs have been bred here, there being a Pekinese Club for the special encouragement of the breed, and the classes at the Kennel Club Show are always remarkably well filled. The classification adopted is for dogs under 10 lbs., over 10 lbs., for any weight, and for biscuit-coloured dogs. At the present time the Pekinese is one of the most 
fashionable varieties of toy-dog, and fabulous prices are asked and obtained for good specimens, whilst the stud fees run up to twenty guineas; but there is no doubt that the ladies will in course of time begin to tire of these foreign toydogs, and as a result the breed will follow the general trend of doggy commerce, and prices become reasonable. The Pekinese exists in a wide range of colours, such as biscuit, chocolate, brindle, black, black and tan, red, white, etc. Fawn with black facial markings is a colour much sought after. The nose ought to be black in red and black-and-tan dogs, but in other shades it is often pink. Some of the special prizes awarded by the Pekinese Club debar a dog from competing if it has a pink nose. Numerous special prizes are offered by this club for dogs under 6 lbs. in weight, hence there is a desire to favour the lighter weights. The under-coat is short and thick, and the outer straight, and there is a very heavy mane. A striking feature of the Pekinese is the heavy conformation of the body in front, as compared with its lightness behind. The head must be large, broad, well domed in the skull, and have a great degree of width between the eyes, which should be prominent and black in colour. The lower jaw must not project beyond the upper, 


\section{POINTS OF THE VARIOUS BREEDS 139}

but take an upward turn to meet the upper jaw. The muzzle must be square, and the markings or black points sharply defined-in fact, the sharper the better. Fore-limbs straight, of proportionate length, with plenty of bone and substance, and an abundance of feather, ending in long toes, well feathered between them. The tail must curl tightly over the back, and be particularly full in the plume, a matter to which Pekinese judges give a good deal of attention. The movements of a Pekinese should be active, and the general expression of the dog vivacious.

\section{Toy Spaniels.}

Under the heading of 'toy spaniels' the following varieties are classified:-

Blenheim Spaniels.

King Charles or Black and Tan Spaniels.

Ruby or Red Spaniels.

Tricoloured Spaniels.

Prince Charles Spaniels.

All the varieties of toy spaniels have for generations been carefully bred, and highly prized, more especially amongst the nobility, and some extraordinary prices have been paid for these small dogs, They are, unquestionably, charming little animals, and, in the writer's 
opinion, much more deserving of popularity than are their rivals, the Pomeranian, Pekinese, and Japanese. Although all these spaniels are very handsome, the ruby and tricoloured specimens are particularly fascinating. The Blenheim spaniel is so-called owing to its association with the Marlborough family at the palace of Blenheim, though it is more than questionable whether the breed originated here. The correct colour is white, with chestnut markings, and the more evenly distributed the markings are, the more highly prized is the specimen. The ears must be long, heavily feathered-in fact, the longer the ears are, the better. Commonly the hair upon the ears is of a deep chestnut, and so are the cheeks, with a white blaze extending from the nose over the forehead. The so-called 'spot,' which is regarded as the beauty spot or hall-mark of perfection, is sometimes present about the middle of this white patch, and consists of a thumb-shaped marking of chestnut. No doubt too much attention is paid, by those who have a superficial linowledge of the breed, to the presence of this mark, and many of the best specimens on the show-bench are without it. The skull should be well domed, and project until it touches the nostril; in fact, there should be no bridge to the nose, the stop 
being deep, and the nose well turned up. Eyes must be large, very prominent, and placed well apart, expressing extreme sympathy and a lovable disposition. The tongue must never protrude from the mouth, nor must the lower jaw project beyond the upper, as in the Bruxelles Griffon, but must be turned up to meet the upper jaw. Neck and shoulders short and neatlyrounded; a compact body, with a broad breast; straight fore and hind limbs, with an abundance of feather upon both. The coat must be long, silky, and straight; the tail ought to be heavily feathered and carried low. The chief faults of the Blenheim are poor markings, projecting under jaw, legginess, want of general compactness, too much nose, defective dome of skull, curly coat, bad carriage of stern, etc. The King Charles spaniels are black, with tan points, and tan spots over the eyes and cheeks. There must be no white hair on the chest; white hair in any part is a disqualifying feature. In tricoloured specimens the black markings must correspond with the red ones of the mane, but the spot is, of course, absent. In all three varieties of spaniel the points are practically identical, and the average weight is from 7 to 10 lbs., but the smallest specimens are those held in greatest esteem. Any one who is going 
in for breeding these dogs, ought to secure a young brood-bitch of medium size, and select as a sire the most typical specimen of the breed that can be found. For this purpose it may be advisable to visit one of the leading shows and make arrangements accordingly. 


\section{FOREIGN DOGS.}

THE term 'foreign' includes any breed of dog that has its origin, or is imported from, abroad, but does not include certain breeds which, though once foreign, are now regarded as British, or those dogs for which the Kennel Club has allotted separate classifications. The writer is inclined to think that many of these importations should have been left in their native lands, and he would like to see a Canine Aliens Bill which would enable the authorities to deport all dogs that the judges declare unsuitable to compete with the many breeds already estab. lished in this country. The dogs that are principally shown in the foreign class are as follows :-

The Samoyede.

The Esquimaux.

The Dingo.

The Bouledoge Français.

The Lhassa Terrier.

The Persian Greyhound. 
The Hairless Dog of Mexico.

The Dhole.

The Chesapeake Bay Dog.

Thibet Spaniels.

Elk Hounds, etc.

Australian Terriers, etc.

\section{The Samoyede.}

These are white dogs having their origin in the Arctic Regions, being there used for sleigh work. They are smart, active-looking animals having a dense, white, stand-off coat, black nose, erect ears, and a keen facial expression. There ought to be a dense soft undercoat, thus affording additional protection to the skin, so necessary in the cold regions. The hair around the throat forms a sort of frill, and the head has a distinctly foxy look. The puppies are very handsome, and, when about six weeks old, strongly resemble a ball of wool, hence many ladies take a particular fancy to them at this age. Both at the Kennel Club Show and the Ladies' Kennel Association Show, there are usually about half a dozen Samoyedes entered, and it is quite possible that each year will see an increase in the number of entries. 


\section{FOREIGN DOGS}

\section{The Esquimaux.}

This breed of dog is employed by explorers and others in Arctic Regions, and it varies in its colour, some being white, others silver grey, or black. In appearance the breed strongly resembles the wolf, the muzzle being long and pointed, and the eyes set obliquely in the orbits, whilst the ears are small, inclined forwards, and erect. They are big, powerful dogs, and the amount of exertion they can undergo is truly wonderful. The coat is dense, and stands off from the body like that of a Chow-Chow, there being a dense, soft, woolly undercoat, and any judge, looking at one of these dogs, ought not to award it a prize unless it is particularly good in coat. The body must be well put together; in fact, to be typical, an Esquimaux dog must be well coupled fore and aft, and well developed in its middle piece. Legs to be clean, big-boned, and of good conformation.

\section{The Dingo.}

This is the native dog of Australia, and in colour it is either red or red interspersed with black hair. The ears are erect, the body long and strongly built; in fact, the dog is heavily built throughout limbs and body, and the back- 
ward position of the hind-limbs indicates that it is particularly adapted for running. The eyes are dark-coloured, the muzzle pointed, the forehead flat, and the neck short and strong. The tail strongly resembles, and is carried like, a fox's. The feet are large.

\section{The Bouledoge Français.}

It is only within the last few years that this breed has been introduced into this country, but, since the formation of the French Bull-Dog Club and the allocation of classes for the breed by the Kennel Club, it has sprung into prominence. The classification of the Kennel Club is for dogs and bitches over 24 lbs., but not over $28 \mathrm{lbs}$. in weight; for dogs not over 20 lbs.; for dogs over $20 \mathrm{lbs}$., and not over 24 lbs.; together with the brace and team classes. The French bull-dog strongly resembles the British breed, and the large ears are the most distinctive features; in fact, it would appear to be simply a bull-dog that at some time or other had been introduced into France and allowed to run riot so far as showbench points are concerned; in other words, a British bull-dog deteriorated through residence in France. However this may be, it has become an established breed in this country, and a few words relating to it are necessary. In general 


\section{FOREIGN DOGS}

build it is practically identical with the bulldog. The colour varies; it may be brindle, smutty, or any whole colour, but admirers of the breed do not like black. Body short and muscular, and the chest deep, whilst the breast should be broad. The head must be large; the forehead flat; and the deeper the 'stop' the better. Muzzle short and broad, and the under jaw longer than the upper. The correct type of ear is the so-called 'bat' ear, which is broad at the base and stands erect. Fore-arms must be very muscular and big-boned, short, straight, and end below in compact feet. The erect carriage of the ears reminds one of the Scottish terrier, because the orifice of the ear is directed forwards. Hind-limbs strong and straight, and dog must be free from any cow-hock conformation. A close coat, a low set-on, short, thick tail, and a general bull-dog conformation throughout may be looked upon as the essential points of the breed. It is unnecessary in a work of this sort to enter into a detailed description of a breed that is not particularly interesting.

\section{The Lhassa Terrier.}

This variety of dog is indigenous to Lhassa, and is useful chiefly as a lap-dog, being about the size of a Skye terrier, but differing in many respects 
from that breed. In colour, Lhassa terriers are sandy, black, dark grizzle, or a blend of these with white. The coat is long and very dense, and the tail is carried over the back. The Lhassa terrier should have a narrow skull, moderately flat on the top, and similar to that of the Skye terrier. The body is of medium length, and the ears are like the drop-eared Skye terrier's. The Lhassa is not likely to find much farour in this country.

\section{The Persian Greyhound.}

These dogs are practically identical with the Afghan greyhound, several specimens of which have been entered in the foreign classes at the Crystal Palace Show. They are built upon racing lines, as our own greyhounds are, but are slightly heavier in build and have long hair on the ears, tail, backs of the limbs, etc. This gives them a very quaint appearance.

\section{The Hairless Dog of Mexico.}

Specimens occasionally make their appearance at the Kennel Club Shows. Very little is known regarding the origin of the breed, but Jesse in his Researches into the History of the British Dog, referring to the dog of Buenos Ayres, says: 'There are also small dogs without hair, except on the 
head and the tail, which are shagged; they are often the companions of the ladies of the country.' In most respects the Mexican hairless dog resembles a black-and-tan terrier that has been shorn of its hair. In weight they are about $18 \mathrm{lbs}$.

The remaining foreign dogs are the Dhole, which is a native of India, and the Chesapeake Bay dog, which is said to have originated on the shores of Chesapeake Bay and its tributaries. It is stated that during the year 1807 an American ship, the Canton, fell in at sea with an English brig in a sinking condition. The American vessel took off the crew, together with two puppies, a dog and a bitch, and the skipper of the ship bought the puppies from a sailor and took them to Baltimore with him. The bitch puppy was black, and the dog red, and through them the breed, it is said, was established. In colour the Chesapeake Bay dog is reddish brown, the coat undergoing an alteration in the autumn; they are about the size of a retriever.

Other foreign dogs are the Elk hounds, Thibet spaniels, Australian terriers, Thibet mastiffs, the last named occupying premier position amongst the foreign dogs at a recent Kennel Club Show; but these breeds are not of sufficient importance to merit any individual description. 


\section{DISEASES AND ACCIDEN'TS}

\section{Distemper.}

This is an exceedingly common disease, and one from which most young dogs suffer, although all dogs are not affected by it. It may be defined as an infectious eruptive fever, communicable directly or indirectly from one dog to another, and also transferable to the ferret family. It is closely allied to influenza, and prostration is characteristic of both diseases. Every year a large percentage of deaths are attributable to distemper-a complaint that may be spoken of as a canine plague, varying in its intensity from time to time. A well-marked attack of the disease, although it does not confer immunity, to some extent protects the dog from a further attack. When adult dogs are affected, the chances of recovery are good, but sometimes distemper proves fatal to old dogs, more especially if lung complications arise. It is a popular, although erroneous, opinion that a dog must necessarily have distemper, and many owners 
mistake an ordinary cold for this complaint. Young dogs frequently contract the disease at shows, probably through the benches being infected. Veterinary surgeons do not admit dogs to shows when they have distemper, and the inspection is usually of a fairly thorough nature, but during the latent phases of the disease it is quite possible that a distemper-affected dog may gain admission to a show now and then. Strictly speaking, distemper must be regarded as a disease specially prone to attack the young, and most dogs suffer from it between the ages of two and nine months, but an attack may come at any time. The course of the disease is considerably influenced by the temperament, constitution, and condition of the animal at the time of attack, and there can be no doubt that surroundings play an equally important part. For instance, a dog, comfortably housed and kept in clean quarters, will be much more likely to pass satisfactorily through the various phases of the complaint than will one kept in unhealthy surroundings.

Symptoms.-Although variable in its manifestations, distemper always presents certain features which serve to distinguish it from allied conditions. During what is termed the 'incubative' period of the malady, i.e. the time elapsing from the date of infection to the development of symptoms, the 
owner may not be aware of aught being amiss with the dog, yet the animal, if carefully observed, will be found wanting in its usual vigour, constantly shivering, and inclined to seek the warmest part of its kennel. During this time the internal temperature will, if taken, be found in all probability to be about $104^{\circ}$ Fahrenheit, the normal temperature of the dog, when at rest and under ordinary conditions, being $101^{\circ}$. If the $\log$ is carefully examined the membranes lying in the eyelids will be found unusually red; the nose dry, yet with a thin watery discharge issuing from the nostrils, the dog occasionally sneezing. The animal looks generally out of sorts, and soon signs of catarrh present themselves. There is a husky cough, and the dog constantly tries to expectorate, but without success. The owner thinks that his dog wants to be sick, but sickness does not occur as a rule, unless artificially produced.

In the course of two or three days the thin watery discharge from the nose changes to a creamy consistence, and this continues until the disease has run its course. The discharge is very characteristic of distemper; in fact, this is the only disease in which it is present. These catarrhal signs, though not absolutely diagnostic of the malady, are almost constant features of it, and by far the most significant, more especially 
when there is a skin eruption, and prostration. Distemper in its simple form is disposed to run a favourable course, but it becomes a deadly scourge when such organs as the lungs, pleura, stomach, bowels, liver, and brain are affected. It is usual to speak of distemper of the head, chest, belly, and so forth, but in all probability these parts are primarily involved in the disease; in fact, it is believed that the specific organisins of distemper, though allied in their bacteriological features, present somewhat different aspects, in accordance with their preference for attacking individual organs. Distemper, appearing as it does in so many obscure forms, is a malady that may easily deceive even an expert, and by the term 'expert' the writer implies a veterinary surgeon. It is these masked forms of the disease that cause so much trouble in diagnosis, and sometimes lead to the dissemination of the complaint throughout a kennel, or, it may be, throughout a whole neighbourhood. It is an ailment that always runs a definite course, and all the so-called 'distemper cures' are either useless or harmful. The malady, by careful medicinal treatment, may be to some extent guided in its course, but never cured, except by the efforts of nature. The skin eruption varies in its severity and in its distribution over the body. It starts as minute red spots, 
which in the course of twenty-four hours increase in size, assuming the form of small pustules. In some cases the eruption, which appears most commonly on the skin of the belly, inside the thighs, and under the arms, but sometimes all over the body, is really the only evidence that the animal has distemper. As in scarletina, shedding of the epidermis takes place, but in a much shorter time, eight days. In addition to the eruption there is a characteristic odour from the skin, a smoll that is casily recognised if it has been smelt once. ${ }^{1}$

A dog, when it has distemper, loses flesh in a very rapid manner, and severe prostration is one of the outstanding features of the disease. In almost every case there is inflammation of the mucous membrane lining the eyelids, and this often leads to a clouded condition of the cornear. As convalescence becomes established, the cloudiness usually disappears, but in some cases it does not do so, the force of the poison again and again attacking the eyes, giving rise to a recurrent form of ophthalmia, generally persisting until complete destruction of the eyeball occurs, leaving the dog permanently blind. In passing, it may be said

1 A condition (often observed in the molar teeth especially) after an attack of distemper is a shedding of the enamel' in patches, exposing the surface beneath. 
that there seems to be no means of curing this form of the disease. The cornea ulcerates, the humour of the eye escapes, the eye heals, or rather attempts to heal, only to be again attacked. Suppuration from the eyes is the result of infection by pus organisms.

Sore throat, bronchitis, vomiting, dysentery, a yellowness of the skin, thirst, paralysis, fits, and convulsions occur, either individually or collectively, in some cases of distemper. Paralysis, however, and chorea or St. Vitus's dance are commonly regarded as sequels to the complaint, and it is quite true that they do not come on, as a rule, until towards the close. These two complications, though not necessarily fatal, sometimes mean death. A dog levelled to the ground through paralysis following distemper, has a better chance of recovering permanently than has a dog with chorea, the complaint last named usually persisting throughout the animal's life.

The details of distemper are so many that the writer has been able to give only a very short sketch of them, and he must now hasten to giveand this is far more important to the reader, if he be a layman-an outline of the proper management and treatment of the patient. If the disease breaks out in a kennel of young dogs, immediately isolate the diseased animal, and tell off a separate 
attendant to look after it, for there is danger of infecting the other dogs through the kennelman's clothes and through the appliances he touches. Always bear in mind that distemper is an acute, infectious, eruptive disease, communicable directly or indirectly, and that this makes complete isolation imperative. A dog may easily contract distemper, whilst at exercise or in the street, and many are infected in this manner; and the writer has known the visit of a keeper from one kennel to another to introduce the disease where it had never been known before. It is always advisable where a number of young dogs are kept together, to separate from the rest any that show the slightest signs of cough, cold, or sore throat. The kennel must be kept scrupulously clean, cleanliness often lessening the severity of the malady; moreover, a filthy kennel has an exhausting influence over the animal constitution. A dog when it has distemper must be fed in accordance with the rules laid down under the heading of 'The Special Feeding of Sick Dogs' (see paragraph). Boiled fish, and, what is still better, a small portion of a salt red-herring given several times daily, will do good; puppies must be kept warm, preferably in a moist atmosphere, best obtained through the use of the fumigating apparatus sold by Messrs. Allen and Hanbury, 
Plough Court, Lombard Street. If the vaporiser is allowed to act in a shut-up kennel, it will exert a most beneficial influence, more especially when the bronchial tubes and lungs are affected. Two or three drops of terebene, and five drops of oil of eucalyptus, blended with a teaspoonful of honey and the same quantity of brandy, given two or three times daily, has generally a very salutary effect, not only in controlling the respiratory irritation, but also acting as a general antiseptic. For the troublesome, husky cough, it is, as a rule, advisable to give an emetic to clear the throat. From one teaspoonful to a tablespoonful or a little more of ipecacuanha wine is suitable for this purpose. It is not generally necessary to repeat the dose. Tablespoonful doses of strong coffee, or doses of from one to four teaspoonfuls of brandy, are beneficial stimulants in distemper, more especially if the latter be combined with some nourishment, such as the yolk of an egg beaten up or a teaspoonful of Brand's essence. A small quantity of tripe is excellent; this can be given two or three times daily. If vomiting is troublesome, give 20 grains of powdered bismuth, combined with 10 grains of bromide of ammonia, in a tablespoonful of water every four hours. When the bowels are in an irritable condition, from 3 to 10 grains of grey powder should be placed on the 
tongue daily, but it must be borne in mind that looseness of the bowels is salutary, and, unless excessive, should not be checked. Dysentery can usually be controlled by the employment of powdered ipecacuanha, say 5 to 8 grains two or three times daily. If jaundice, commonly called 'the yellows,' is a marked symptom of disease, give from 2 to 4 grains of calomel on the tongue every other day, unless the drug acts too freely as a purgative. In cases of this nature it is advisable to allow dogs soda-water and milk, or, what is equally beneficial, milk-whey; lime-water is also of service. Rub the throat, if it is sore, as will be indicated by a difficulty in swallowing, with white oil, and the chest with camphorated liniment; but when lung complications are suspected, it will be better to apply hot linseed and mustard poultices around the front and sides of the chest, fastening the poultices on with a broad flannel bandage. For the treatment of fits, see 'Epilepsy'; also see paragraphs on 'chorea' and 'paralysis.' Sponge the eyes, nose, etc., several times daily with a little cold tea, avoiding the use of all substances such as milk, a dirty sponge, etc. As a dressing for the eyes a little boracic ointment is most useful; smear a small piece between the eyelids twice daily. All cases of distemper, no matter of what degree, call for a great deal of attention, 
and, unless the owner is prepared to devote time and a reasonable amount of expense, a successful issue cannot be hoped for. It is a popular, but erroneous, idea amongst laymen, that a dog should be starved, or rather kept short of food, whilst labouring under distemper. This will complete the subjugation of the animal, but not of the disease, and the owner of a dog or horse that is attacked by distemper should remember the old proverb that 'a righteous man regardeth the life of his beast,' which is not embodied in starvation fare.

\section{Inflammation of the Lungs and Pleurisy.}

The dog, like all other animals, is occasionally a sufferer from disease of the lungs and their covering (pleural membranes), and occasionally the inflammation extends to the serous membrane enclosing the heart (the pericardium, or heart sac), resulting in the production of what is technically termed 'pericarditis.' In all probability lung inflammation is always the result of infection by micro-organisms, the commonest of these being the organisms of distemper. But pneumonia appears in the dog quite apart from this malady. If this disease is suspected-indicated by quickened breathing, general dulness, high temperature, thirst, and a disposition to keep sitting, with the nostrils in the direction 
of a draught,-it will be best to obtain the services of a veterinary surgeon, because, unless this malady is properly treated, the animal will certainly die.

\section{Bronchitis.}

By bronchitis we mean inflammation of the bronchial tubes. This, when it attacks the larger air tubes, is not of such gravity as when the smaller ones are chiefly implicated, there being in the latter case a danger that the disease will lead to inflammation of the lungs, or to what is known as catarrhal pneumonia. Bronchial inflammation is commonly present in distemper, in fact, usually part and parcel of that malady. Its appearance in these circumstances is favoured by neglect, or by keeping the animal in a damp kennel, dampness being an exciting cause. It is usual to speak of bronchitis as 'acute' and ' chronic,' the one often terminating in the other, but acute bronchitis is the form usually met with in the dog. The so-called 'mechanical' bronchitis is that arising from the inhalation of irritating vapour, whereas the prefix 'parasitic' is employed, when the bronchial inflammation has arisen through the presence of threadworms in the air-tubes.

Srmptoms.-The leading symptoms of bronchitis are the so-called ræ (a sound produced by air 
passing through fluid in the tube), fever, and frequent attempts at expectoration.

Treatment and Management.-Keep dog in a warm house; clothe body; use the fumigating apparatus recommended under 'distemper'; poultice chest if necessary; and give 20 drops of ipecacuanha wine, combined with 10 drops of tincture of belladonna, and 5 drops of concentrated liquid acetate of ammonia, in a dessert spoonful of water, every six or eight hours.

\section{Catarrh or Coryza.}

In plain language, catarrh means a cold, affecting the mucous membranes of the head, and indicated by watery discharge from the eyes and nose, sneezing, and general dulness. Catarrh, as previously stated, nearly always accompanies distemper, and it is usually a very difficult matter for a layman to differentiate between that ailment and the benign condition now under consideration. As a rule, two or three days will usually settle the matter. Catarrh is not infective; does not prostrate; is not accompanied by any skin eruption; ends as simply as it began; and requires nothing beyond good general nursing.

\section{Epilepsy.}

Fits or convulsions, both in adult and young 
dogs, more especially the latter, are of common occurrence.

In distemper, convulsions frequently come on, diminishing the animal's chances of recovery. In all probability irritation in the alimentary canal (worms, etc.) brings on fits, if the germs of the malady are in the blood.

The convulsions of distemper are somewhat different from the epileptic seizures seen in the adult animal, being in the former case, in the author's experience, more frequent, of shorter duration, and less disposed to leave the sufferer in an unconscious or semi-conscious, condition.

External injuries to the cranial vault are sometimes, but not often, the cause of fits. The fits that come on during distemper often follow one upon the other at intervals of from half an hour to several hours or, it may be, several days. There is nothing to guide one as to the time when a seizure may take place.

Excitement is liable to produce a fit. Experimentally, fits have been produced by stimulating with a galvanic current certain convolutions of the brain. The duration of the convulsions is seldom above a few ininutes, or, it may be, only a few seconds.

The chief points to attend to in these diseases are :- 


\section{DISEASES AND ACCIDENTS}

(1) To feed on easily digested food.

(2) To keep the bowels moderately loose.

(3) To avoid all exciting influences.

(4) When the fits are on, to pour a little cold water over the head.

(5) Give internally 10 to 20 grains of bromide of potash night and morning in a tablespoonful of cold water.

Adult dogs should be treated for worms.

\section{Chorea (St. Vitus's Dance).}

This morbid process is one of the worst sequels to distemper, often retarding or preventing what promised to be good recoveries from the original complaint. It is also known as twitch.

The presence of chorea is denoted by the twitching of certain muscles, more especially in the region of the head, though by no means uncommon in other parts, such as the legs, etc. If but slight, chorea may escape observation for a long time, whereas in other cases the malady is so pronounced as to interfere with the animal's usefulness. These involuntary twitching movements are, in some instances, in abeyance during sleep, becoming apparent immediately the dog is disturbed.

When purchasing a dog, particular attention 
should be paid to see that it is free from the complaint, more especially if it has recently had distemper. With reference to treatment, a course of alterative and tonic medicine, associated with malt and cod-liver oil, is the most suitable. A very useful remedy is to give a teaspoonful of Fellowes' Syrup of Hypophosphates night and morning. Easton's Syrup may also be tried. The best of food must be given. If the malady is severe, the chances of cure are small.

\section{Paralysis.}

This can either be local or general, i.e. a certain part may be thrown out of action, or the whole of the body 'apparently' paralytic. Mere want of control over the hind-quarters, such as the debility observed, so commonly, in this region after distemper, must not be confused with the condition now under consideration. In apoplexy of the brain, or in the case of an injury to the spine, such as may happen when a dog has been run over, the animal is suddenly paralysed. Loss of consciousness necessarily means loss of motorpower over the whole body. A broken leg, jaw, etc., renders the part useless or paralytic until there is a reunion of the parts injured. In strychnine poisoning the tetanic spasms commonly render the animal completely paralytic. Disease 


\section{DISEASES AND ACCIDENTS}

of the spine, of the textures enclosing it, or of the brain is a frequent cause of paralysis, either 'complete ' or ' partial.' Treatment can be successfully applied only when the likely causes have been considered. A frequent cause (not effect) of paralysis in the dog is constipation. If this is the case, give a dose of purgative medicine and an injection of soap and water or glycerine, say two tablespoonfuls. If due to rheumatism, treat accordingly. In this, as in many other maladies, the author advises that a 'qualified' (M.R.C.V.S.) practitioner be called in. We emphasise the word 'qualified,' because the authormuch to his regret-has had it brought to his notice that in many instances the unqualified sons of veterinary surgeons have professed a knowledge of canine diseases, and by mistakes due to their youth and ignorance (to say nothing of their impudence) have brought disrepute upon qualified and able practitioners. Consultants should beware of sons working under the title and qualifications of their fathers.

\section{Apoplexy.}

An apoplectic stroke is denoted by the suddenness of attack, the dog being struck to the ground without the slightest warning, becoming unconscious, and perhaps dying immediately. In epi- 
lepsy (falling sickness) the dog is convulsed, the muscles of the face, eyes, jaw, body, etc., all being involved, whereas in an apoplectic stroke from the brain, the animal does not show these signs. If apoplexy is suspected, give the dog a quick purgative and act as advised in paralysis.

\section{Rheumatism.}

(Also called Kennel Lameness and Chest-founder.)

Rheumatism, both in its acute and chronicmore especially the latter-forms, is a fairly common complaint of the dog, and one for which most quack-medicine vendors have their 'socalled' cure. 'Tis fortunate for some-unfortunate for others-that the dog has not the power of speech. The causes of rheumatism are of a 'predisposing' and 'exciting' nature. Hounds are very subject to it, and sporting, more frequently than non-sporting, dogs. Some dogs seem to have an attack of rheumatism when the slightest exciting cause (damp, cold, etc.) is present. Easterly winds have been known to produce it.

Syмртомs.-Generally constipation, tenderness when the loins are manipulated (lumbago), or complete loss of use in hind-limbs. When rheumatism is confined to the fore-quarters- 
chest-founder-the dog screams when made to move about. Sometimes a dog will give a sudden howl when moving, and the owner fails to detect any cause for it. The possibility of rheumatism should be borne in mind.

Treaturent.-Give a 5-grain blue pill until the bowels are freely open. Rub the parts with the following liniment night and morning :-
R Opodeldoc, . . . 4 ounces Capsicum liniment . 4 ounces
Mix, and use as directed above.

If the malady is 'acute,' give twenty grains of salicylate of soda night and morning, but if 'chronic, five grains of iodide of potash in one tablespoonful of water. As to food-fish, milk, bread and meat, and soda-water to drink instead of ordinary water; when the dog becomes thirsty he will drink soda-water.

\section{Rabies (Madness).}

For the absence of rabies in this country thanks are due to the muzzling order and the strict quarantine regulations. In certain other countries rabies continues to rage, deaths from hydrophobia occurring from time to time. As many of our readers doubtless reside in foreign lands where the malady prevails, it is desirable to make brief reference to some of the more important features 
of this deadly canine ailment. First of all, the bite of a rabid dog or other rabid animal is liable to be followed by hychrophobia in man, or rabies in other animals, such as the horse, wolf, cat, ox, tiger, pig, ete. Secondly, the disease assumes two distinct forms in the $\operatorname{dog}$, (a) furious rabies; (b) dumb rabies. In the form last named the characteristic sign is found in a 'dropped' or paralytic condition of the lower jaw, the tongue hanging out. An early symptom of rabies is shyness and restlessness, the whole disposition of the $\log$ being altered. He becomes peevish, snapping at imaginary objects, and, later on, at other animals coming in contact with him. $\Lambda$ marked feature, usually present, is biting, licking, or tearing the original seat of injury-the inoculation wound. There is an alteration in the voice - a somi-bark and howl-and this is very characteristic. Later on, there is inability to swallow water, with an inclination to gnaw at anything, particles of wood, straw, ete., being usually discovered in the stomach post-mortem. All these signs rapidly increase in severity, others, of an irregular nature, commonly showing themselves towards the close, which is seldom prolonged beyond the seventh day from the onset of suspicious signs. From one to eight weeks-though in some instances several months-may be taken 
as the time elapsing between inoculation and the development of symptoms. Three months is the time fixed for quarantine, so that this may be taken as the maximum time for isolating an animal bitten by one suspected as rabid.

\section{Indigestion (Dyspepsia).}

Indigestion means perverted assimilation, and, simple as the malady may appear to be, its presence suggests much, for the disorder may arise through functional or organic changes in any part of the body, and not merely in the stomach or bowels. Purely functional disorder of the stomach, no doubt, does occur, and is capable of producing dyspeptic symptoms. Decayed or decaying teeth are frequently found in dogs, and the irregular action of the bowels, skin disease, hair falling off, and an offensive breath are sufficiently indicative of indigestion. Worms in either stomach or bowels, or in both, are frequent causes. Vomiting is often present. An effort must be made to ascertain the cause, or probable cause. As powders for indigestion, try the following :-

\begin{tabular}{|c|c|}
\hline Be Pepsin . & . \\
\hline Charcoal & . \\
\hline Carbonate & of bismuth \\
\hline
\end{tabular}


Bicarbonate of soda . 2 drachms

Powdered rhubarb . 24 grains

Mix, and divide into one dozen powders.

Directions.-Give one powder, just as it is, night and morning, immediately before food. Feed on rice and milk, boiled fish, boiled sago, bread and water, potatoes with meat finely cut up.

\section{Gastritis.}

\section{(Inflammation of the Stomach.)}

By far the most frequent-and gastritis is a common disease amongst dogs - causes of inflammation of the stomach are irritant poisons and worms. Most rat poisons will readily produce gastritis. Arsenic and antimony are frequently used in a criminal manner.

Sympтoms.-Thirst, vomiting, pain in the belly, superpurgation, and prostration are the leading symptoms of this, usually fatal, malady. In most cases it is safe to give an emetic, but subsequent treatment should, preferably, be left- to the professional man.

\section{Enteritis.}

\section{(Inflammation of the Bowels.)}

Inflammation of the bowels arises through a variety of causes, the chief being twist of the gut, 
intussusception of a portion of it, various chemical agents, such as arsenic and antimony, etc., worms (a very common cause in puppies), and external injuries. Stoppage of the bowels, either through habitual constipation or the presence of a foreign body, sometimes produces inflammation. Pain in the belly and vomiting are the most suspicious signs. A large percentage of puppies die through enteritis set up by round-worms (Ascaris marginata). They begin by refusing to suck, and are constantly crying from the pain, and nearly always die. As a preventive, give the bitch several good doses of worm medicine before she comes in season. Habitual constipation can be overcome by giving twenty drops of sacred bark daily. Hot fomentations to the belly, and fifteen to twenty-five drops of chlorodyne should be given to adult dogs, every four hours, in water, but, owing to the grave nature of the malady, professional aid ought to be obtained; besides, treatment must be in accordance with the cause.

\section{Diarrhœa.}

Strictly speaking, this is but a symptom of some derangement of the alimentary canal, being nature's effort to rid the system of offending materials. Many and varied are the causes excit- 


\section{THE DOG}

ing the stomach and bowels to discharge their contents in a fluid or semi-fluid condition. In distemper a fetid diarrhœa is common, and usually of a very intractable nature. Worms are a fruitful cause, so that, if there is the least suspicion of these parasites, it is generally a good plan to give a dose of worm medicine. Exposure to cold, and prolonged feeding on unsuitable food, e.g. oatmeal and liver, are very liable to bring on diarrhœa. In such cases a dose of ordinary diarrhœa mixture, as sold for the human being, will generally check the discharge. From 10 to 20 drops of chlorodyne will cure mild cases. It should be given, night and morning, with 20 grains of bicarbonate of soda and the same quantity of prepared chalk, added to a tablespoonful of water. The food ought to consist of boiled arrowroot and milk, milk and rice, raw eggs.

\section{Dysentery.}

Dysentery is a disease principally of the large bowel, the gut becoming ulcerated in parts. In some instances it assumes a chronic form. The causes are variable.

Symptoms.-Pains in the belly, straining; blood mingled with mucus and freces, is passed until the bowel is emptied; straining and passage of 
blood, etc., is repeated. Loss of appetite and shivering are additional signs.

Treatment.-Begin this by washing out the bowel with a warm-water enema-say one to two pints, to which a teaspoonful of hazeline has been added. Keep the dog perfectly quiet afterwards. Now give it the following draught:-

B Powdered ipecacuanha, . . 20 grains Bicarbonate of soda. . . 20 grains Syrup of lemon, . . . $\frac{1}{2}$ ounce Mix.-Repeat in eight hours, and gradually decrease the dose as recovery takes place.

Food.-Much the same as that recommended for diarrhœa.

\section{Poisons.}

For the rapid destruction of a dog, inject Scheeles' Prussic Acid, but this must be done under professional supervision. We recommend this method of destruction, it being certain, safe, and speedy. Destruction by chloroform is too slow, the struggles of the animal preventing a quick result. The lethal chamber, or the use of coal gas in a closed chamber, constitutes an excellent means for destroying dogs. The criminal, and often the accidental, destruction of dogs is generally effected by means of arsenic, strychnine, antimony, or rat poisons. Strychnine poisoning is 
denoted by the sudden seizure and spasms of the muscles. These muscular contractions are generally very severe, extending over the whole of the body. As soon as ever the drug enters the stomach, there is a rapid development of tetanic spasms, and the dog may die within a few minutes-fifteen or twenty-if the dose has been large. Strychnine is a white crystalline solid, and only infinitesimal doses can be given medicinally. If the dog survive long enough, make an infusion of tobacco-say a couple of ounces of shag-tobacco to a pint of water-and give two to four tablespoonfuls of this every two or three hours.

\section{Eczema.}

Eczema is an acute or chronic inflammation of the superficial structures forming the skin, and is produced by chemical, mechanical, or constitutional causes, the latter being apparently of an hereditary nature. The kennel terms 'blotch,' 'red mange,' and 'surfeit' often cover eczema.

In its acute form the disease shows itself either as reddened patches with a moist surface, or as a generally diffused moist condition of the skin, this condition being particularly evident under the fore-arms, and between the thighs. When chronic, as it commonly is on the dog, eczema 
often makes its appearance around the margins of the ears, and on the eyelids, points of the elbows; also on the buttocks and hocks, when it is spoken of as ' psoriasis,' indicated by a dry and scaly condition of the affected area. In ordinary ' mange,' or rather in mange brought on by parasites, eczema is induced; in fact, eczema represents the mangy condition.

It must be admitted that the causes of eczema are, as a rule, of a very obscure nature. The eruption is the outward sign of inward disturbance, and the liver, the kidneys, the nervous system, the digestive system, etc., are, in many cases, at the root of the whole mischief, and until these organs have been restored to healthy activity, no cure can be attained. The reader must bear in mind that in nine cases out of ten (except when caused by parasites) eczema is the result of impaired constitutional vitality, and treatment must be directed towards building up the constitution. External applications, though useful, are not so important as medicine taken internally. The former, when used alone, merely patch up, and the repair is only of a temporary nature. Although it appears highly probable that eczema is hereditary, it is not infectious. In this respect it stands out in marked contrast to parasitical mange. The chief difficulty confronting the non- 
professional in the diagnosis and treatment of eczema, and other skin affections closely related to it, is the trouble in detecting the presence or absence of mange-producing parasites. External applications, capable of acting beneficially in the one case, may not be of much service in the other, whilst in mange there is really no necessity for internal medication. Under these circumstances, the writer advocates that, if possible, professional assistance be sought.

Where there are a number of dogs kept together, it will be advisable to keep the infected animal separate until the owner has satisfied himself that the disease is not contagious. This being settled, treatment may be begun. If the eruption is acute, apply an ointment composed of 2 ounces of boracic acid, a drachm of oil of tar, and a couple of drachms of red oxide of mercury. In the moist or 'weeping' form of eczema the surface must be protected from atmospheric influences, which can be done by the application of a cream, consisting of chalk and lime-water, or oxide of zinc and lime-water. This application should be used several times daily, and to prevent the dog from licking it off, a muzzle, or the so-called Elizabethan collar, which is made so wide that the dog cannot turn its head round, has sometimes to be used. For chronic eczema on the ears and around the 
eyelids, rub with tar ointment night and morning; for eczema on the points of the elbows, etc., dress with Chrysarobin ointment daily, rubbing it well in. The latter must never be used upon the face or close to the eyes. Internally, give a blue pill, and follow up with 5 to 10 drops of Fowler's solution of arsenic, night and morning, in food, arsenic being specially suitable for chronic cases of eczema. As a rule it is advisable to act on the kidneys, for which purpose give a few doses of acetate of potash, say 20 grains once or twice a day. Build up constitution with cod-liver oil, Fellowes' syrup of hypophosphates, malt extract, etc. Another very useful remedy is Parrish's syrup, and, if half a drachm of Fowler's solution of arsenic be added to a four-ounce bottle of this chemical, a capital mixture for the treatment of eczema is produced. Feed on meat and bread, and give plenty of exercise, avoiding the use of all stimulating foods, salt, etc.

\section{Parasitic Mange.}

The dog, unfortunately for itself, and occasionally for man, is subject to parasitical affections of the skin, and the chief of these are sarcoptic mange, and follicular mange. (The follicular parasites, although, it is said, they are constantly on the faces of some persons, do not produce any 
skin disease in man.) The commonest form of mange infesting the dog is that produced by the sarcoptes mange-mites. These parasites produce circumscribed patches of inflammation upon the skin, but, if the disease is allowed to go unchecked, it will spread all over the surface of the body, and render the animal an object of sympathy and misery.

Any man who allows his dog to get into this condition ought not to be allowed to have the charge of animals. It is obvious that parasitic mange will be communicable from one dog to another, either directly, by the animal itself, or indirectly, by things that have come in contact with it, and the greatest care must be taken to prevent infection. 'Sarcoptic' mange mites live upon the superficial parts of the skin, therefore it is not, as a rule, a difficult matter to reach them with drugs; nor are they difficult to destroy. It is the female Acari that cause the irritation.

\section{Follicular Mange.}

(Black Mange.)

This form of mange is much worse than the other, for the mites take up their lodging in the follicules or roots of the hair, and are very difficult to reach. The parasites are termed Demodex Folliculorum, and, according to Erasmus 
Wilson, they are present in the hair follicules of most men. Follicular mange, though infective, is less so than the sarcoptive variety, owing to the isolated position of the parasites, which, if present, are easily recognised on microscopic examination, although they exist in several forms. When in the adult state, the full-grown mite is clubshaped, and has four legs on each side. If neglected, this form of mange will spread over the whole surface of the body, though not as rapidly as the sarcoptic. In order to detect the parasites, the mangy spot should be squeezed with the fingers, and matter will issue from the inflamed follicules. This material should be spread upon a glass slip, to which a drop of glycerine has been added, and then examined with the microscope.

Treatment.-As a rule follicular mange is a remarkably difficult disease to cure, and in nine cases out of ten will baffle all attempts to do so. The hair should be clipped off all over the body so as to facilitate the application of dressing, which will require to be varied from time to time. Treatment does not always result in a cure, and if the dog is not very valuable, perhaps it is better to destroy him than to waste time and money in what is little better than a forlorn hope. Consult an expert, and be guided by his advice. 


\section{Lice, Fleas, and Ticks, etc.}

The dog is frequently troubled with these pests, all of which cause a considerable degree of irritation, and, where a number of dogs are kept together, often a good deal of annoyance. The best bedding material is straw, but fine shavings are useful for keeping dogs fairly free from fleas. A very good way to prevent the fleas from coming is to wash the dog frequently and to sprinkle the bedding with a mixture of oil of eucalyptus and turpentine. Insect powders are worse than useless. Lice are far more troublesome to get rid of than fleas, and are generally due to bad management; in fact, the presence of lice upon a dog, or a litter of puppies, cannot be regarded as a testimonial to the kennelman or the owner. In order to free a dog from these pests, it is necessary to dress it all over, and an excellent dressing for this purpose is a liniment composed of one part of paraffin oil to 6 or 8 parts of olive oil. As an alternative dressing a liniment composed of half a pound of flowers of sulphur, two ounces of oil of tar, and a pint and half of train oil, answers admirably. After a dog has been thoroughly dressed, it must be put into a bed of clean straw, otherwise reinfection may occur, and the application prove unsatisfactory. All bedding, benches, etc., where- 
ever the dog has been, should be thoroughly scrubbed with hot water and a strong decoction of tobacco. The eggs or nits, as they are called, are much more difficult to destroy than the parasites. The dog tick is a troublesome pest, and becomes a true blood-sucking parasite, increasing to the size of a fairly large pearl, which it is somewhat like. The tick, which usually comes from the long grass and brushwood, is most frequently met with in sporting dogs, and the insects sometimes drop on to the kennel floor, where they will breed. In order to get rid of them the kennel should be thoroughly scrubbed, and those upon the animal touched with paraffin oil, which will cause them to fall off. This method is vastly superior to removing them forcibly.

\section{Worms.}

The dog is often troubled with worms, both round and flat. There is also a flute-shaped parasite that sometimes inhabits the nasal chambers, giving rise to a chronic discharge from the nose, attacks of sneezing, etc. The principal roundworm infecting the dog is that known as the Ascaris marginata, which takes up its abode in the stomach and bowels, and, if numerous, gives rise to general unthriftiness, vomiting, irritation of the skin, and, in puppies, to inflammation of 
the bowels. In fact, a very large proportion of suckling puppies die from this cause within a few days of their birth. In certain countries minute threadworms infest the cavities of the heart, and often cause sudden death, but, fortunately, British dogs are very rarely infested with these worms, the Filaria ammitis. A most important worm is that known as Tcenia conurus, which is the mature form of a larval form resting upon the brain of the sheep, producing in the animal the so-called 'gid' or 'sturdy.' The tapeworm that troubles the dog most is the Tania serrata, which exists in a larval form in the viscera of hares and rabbits. Another species of tapeworm commonly found in the dog is known as Tania marginata; and several other varieties are occasionally met with.

Symptonis and Treatment.-When dogs are in backward condition, their hair falling out, and the appetite being capricious, the presence of parasites within the alimentary canal suggests itself as a cause. At any rate, it is always advisable to adopt what we may term speculative treatment, that is, to have a shot at the worms, with some drug or drugs that will clear them out of the system. Drugs that kill worms are called vermicides, and those that cause their expulsion vermifuges, and it is a capital plan to combine 
the two. All the older remedies, such as tobacco, powdered glass, and other irritants should not be given, these being too irritating to the alimentary canal. Areca-nut is an old and well-tried remedy, and it has proved its value beyond all question of doubt. If it is properly administered, and in sufficient doses, it is a very satisfactory remedy for both round-and tape-worms, more especially when given in combination with a little santonin and a few grains of jalap. The proper mixture is from 5 to 60 grains of areca-nut, 1 to 10 grains of santonin, 5 grains of jalap, and an ounce of milk; the whole can be made into a bolus with butter, and should be given to the dog after a fast of from 12 to 24 hours. For tapeworm kamala is a capital remedy, and may be given in slightly larger doses than the areca-nut. All worm medicines may be repeated in a week or ten days' time.

\section{Bareness around the Margin of the Ear.}

Very often the flaps of the ears will be found bare and in a dry scurfy condition. This is a chronic form of eczema, and must be treated in accordance with the principles laid down under the heading 'eczema.' 


\section{Inflammation of the Eyes.}

The membranes lining the eyelids, either of one or both eyes, may be the seat of acute inflammation. This is known as ophthalmia, and, if it is neglected, it is liable to end in opacity of the cornea, and will either partially or completely obscure the sight. Reference to this has already been made in connection with distemper, but ophthalmia occurs quite apart from that disease, being easily provoked by chemical and mechanical agencies. A diseased condition that sometimes affects the dog is known as inversion of the eyelid, in which the eyelid turns inward, and the eyelashes sometimes grow against the ball of the eye. The very opposite of this also occurs, the eyelid being turned outwards. Both abnormalities necessarily predispose to ophthalmia.

Symptoms.-One or both eyes will be found weeping. Tears flow over the face, scalding the margins of the eyelids; the eye itself is intolerant to light; and the cornea becomes opaque.

Treatment.-Bathe eyes several times daily with an infusion of chamomile, and, after this, apply, two or three times a day, a lotion consisting of 2 or 3 grains of corrosive sublimate and 8 ounces of water, or a solution of boracic acid. When the cornea becomes cloudy, a solution of 
nitrate of silver (4 grains to an ounce of distilled water) will be found of considerable benefit. In other cases the treatment will, of course, vary in accordance with the cause.

\section{Blindness.}

Aged dogs frequently become blind through cataract, and this condition may arise through a variety of causes. For instance, the cornea may be affected, or the crystalline lens, the chambers of the eye, or the nerve of sight. Blindness is either temporary or permanent, and here again the treatment is suggested by the cause.

\section{Internal and External Canker of the Ear.}

By external canker is implied a wound, upon the margin of the ear, that shows little or no inclination to heal, owing to the cartilaginous structure of the ear. In reality, it is not a cankered condition at all; but internal canker, on the other hand, consists of an inflamed condition within the passage of the ear, and is accompanied, in many instances, by an offensive discharge, and, in advanced cases, by injury to the inner mechanism of the ear. All dogs are liable to be affected with this complaint, which sometimes is a mere redness, and, at 
others, gives rise to suppuration, a discharge of blood, and the most offensive odour. One or both ears may be affected; if only one, the dog turns its head towards the affected side; if both, now to this side and now to that. There is also a parasitical form of canker of the ear, with little or no tendency towards suppuration, but a careful inspection within the auditory passage usually reveals a considerable degree of redness and the deposition of a sooty-like material within the recesses of the ear; and the careful inspection of this, with the aid of a powerful pocket-lens, will, in all probability, disclose the presence of minute parasites, characteristic of this particular variety of canker, which is regarded as communicable from one dog to another. Eczema doubtless predisposes to canker of the ear, which, in this case, demands constitutional treatment for its cure. The chances of one dog infecting another are comparatively small, owing to the situation in which the parasites are confined. Although all dogs are liable to suffer from this disease, the long-eared varieties, such as retrievers, spaniels, and toy spaniels, etc., appear to be those most frequently affected. No doubt there are many irritants capable of producing internal canker of the ear, more especially if the animal has an eczematous predisposition. Like 
other forms of eczema, ear canker has an inclination to recur; in fact, it is a difficult matter to know when the disease has been completely cured.

Treatuent.-This will depend on the causes operative in the production, and upon the stage the disease has reached. If there is mere redness and a slight degree of moisture present, the introduction of a little antiseptic powder will often be sufficient to cure it, or, at any rate, to do away with the irritation. At the same time it is advisable to give the dog a course of alterative medicine, such as 5 or 6 drops of Fowler's solution of arsenic, night and morning, in food, together with 5 drops of liquid extract of sacred bark, either in food or water. It is generally advisable to syringe the ears out with a little spirits of wine, which will dissolve any greasy material; this should be done daily. When there is any offensive discharge, and the disease has been in existence for any length of time, there is no remedy superior to an alcoholic solution of chloride of zinc, of a strength ranging from 5 to 10 grains to every ounce and a half of methylated spirit. A small quantity of this should be poured into the ear daily. A little calomel, inserted into the recesses of the ear, and followed by the pouring in of a teaspoonful 
of lime-water, is another capital cure for canker of the ear, if of a non-parasitic nature. A little boracic ointment, carbolic acid ointment, or any other antiseptic cerate is of service in the treatment of canker of the ear. For external canker, the margins of the wound must be thinned down by rubbing them with a little bluestone, and the sore dressed daily with Venice turpentine.

\section{Dropsy of the Flap of the Ear.}

This is a very common form of injury, and appears to be the result of a bruise. It is in the long-eared varieties that the swelling attains its greatest dimensions, but the swelling is proportionately large in shorter-eared dogs. The fluid accumulated represents the inflammatory product, the result of the injury. The swelling bulges both inside and outside the ear, and causes the animal a great deal of inconvenience, though it is not a particularly hot swelling; such pain as there is, arises through the tension on the skin.

Treatuent.-The swelling must be opened, so as to let out the watery fluid. Take a sharp penknife and slit the swelling up, ${ }^{1}$ to the extent,

${ }^{1}$ Make the cut lengthwise, and not across the flap of ear. 
say, of an inch; then insert the finger into the wound, so as to remove any blood-clot and to break down adhesions that have formed. This done, wash the cavity out by syringing it with one part of iodine liniment and three parts of water; keep wound open for two or three days, and bring pressure on to flap of ear by bandaging it to side of head. Instead of making a single cut lengthwise, a cross-shaped one may be made, which, on healing, will produce equal contraction on all sides, thus diminishing any tendency to alteration in the carriage of the ear.

\section{Wounds, Burns, Scalds, Etc.}

A wound may be lacerated or torn, punctured, incised, or clean-cut, poisoned, and so on, but the most important points to note in connection with it are the position, the duration of existence, and the degree of penetration. In dogs, the external wound may be slight, although the laceration within is extensive. This often occurs when dogs have been severely fighting, and the writer has frequently, in a professional capacity, noted injuries of this class, many of them proving fatal. Particular attention should be paid to such wounds. Under favourable circumstances the dog's flesh heals rapidly, but if the animal is allowed to lick a wound, the healing is retarded, 
although the contrary is pretty generally believed. Wounds in juxtaposition to vital parts usually call for professional attention, as inflammation of important organs sometimes arises. The sinews of the limbs, if they have been cut across, will heal, provided that they are treated correctly. The pads of the feet and between the claws frequently get injured, being cut by something sharp on the ground, and an injury here, if neglected, sometimes leads to fistula of the claw. Superficial wounds about the feet can be painted with Friar's balsam, and then bandaged, but if deep, clean-cut, and on the pad, it is advisable to sew them up with stout silk. A tear in the region of the eyelids or face should be sewed up with fine silk, but not until the hair has been clipped off all round the wound, the bleeding arrested, and the part cleansed with some weak antiseptic solution. When there is a broken bone in addition to a wound, the most economical plan is to have professional advice. Burns and scalds require the scalded surface to be protected from the air. First of all, paint the burn over with iodine liniment, and then freely wet with a cream composed of whiting and lime, taking particular care to prevent the dog from licking the injured part. Excessive bleeding from a wound can be controlled by pressure, by the 
application of cold water, or the alternate use of hot and cold water; in other cases, when an artery has been cut across, the best plan is to tie a ligature around it, on the side nearest to the heart; a piece of silk or catgut can be used for this purpose.

\section{Broken Bones or Fractures.}

The dog, like other animals, is liable to be injured upon any part of its bony framework; the injury being a fracture, a dislocation, or a combination of the two. Fractures of the bones of the spinal column always prove fatal, if not at the time of the injury, subsequently. The same remark applies to dislocations in this region. The principal cause of injuries to the spine is being run over, but it is astonishing how a dog will sometimes escape a fatal or even the slightest injury, although the vehicle has passed right across the spinal column. The bones of the limbs are frequently injured, more especially the fore-arm, the arm, the first and second thigh, and below the pastern joints. The principle of treatment in all fractures is practically the same, but a difficulty is often experienced in making a correct diagnosis. The chief signs of fracture are: Crepitation, i.e. a grating of the fractured ends of the bone, particularly evident when dis- 
placement has occurred; swelling; shortening or lengthening of the limb. The dog is unable to bear any weight upon the limb, and manipulation causes pain. Deformity of the limb is not necessarily a sign of fracture; fracture of the metatarsal, metacarpal, and phalangeal bones, unless several of these are broken at the same time, does not produce deformity. Fractures are spoken of as 'simple,' 'compound,' and 'comminuted,' In the first-named the bone is simply broken across, or, it may be, obliquely; in the second, there is the wound as well as the fracture; whilst the third class of injury is denoted by the bone being broken into a number of fragments, and if there is a wound in connection with the broken pieces, it is referred to as a 'compound comminuted fracture.' By the term 'dislocation' we mean that a bone entering into the formation of a joint has slipped out of its natural position, either partially or completely. In the dog, dislocations occur most frequently at the shoulder-joint, the elbow-joint, the toes, and the stifle-joint, but dislocations are not of common occurrence in the dog; when they do occur, the bone must be restored to its normal position, or, in technical language, 'the dislocation must be reduced.' In fractures of the long bones of the limbs, the broken pieces should first of all be brought into 
their normal position, and maintained there by means of splints or bandages; it is a good plan to apply the bandages directly to the skin. A simple form of bandage is made by soaking a length of cotton in boiled starch, rolling up the bandage tightly, and then applying. White of egg can be substituted for the starch, or, if preferred, glue, plaster-of-Paris, etc., the last-named bandage being excellent. Spread dry plaster-of-Paris over a bandage of the requisite length and width, then soak it in water for two or three minutes, and apply. Splints made of gutta-percha, wood, tin, zinc, leather, etc., are often employed; if so, the splint must extend several inches above and below the injury. Gutta-percha splints can be moulded to suit the injured part by soaking the material in hot water. Another useful material is poro plastic, which can be moulded, in the same manner, into the requisite shape. When there is swelling, the bandages must not be applied too tightly, but discretion exercised, because the swelling may continue to increase for a time, and subsequently decrease, the bandages having to be adjusted accordingly. If there is a wound, leave it uncovered, but dressed with some antiseptic; the dog must be kept at rest for six weeks, unless it takes particular care of the injured limb, which most dogs do. 


\section{N DEX}

Airedale Terriers, 113.

Anatomical Outlines (Elementary), 28.

Apoplexy, 165.

Australian Terriers, 149.

Basset Hounds, 53.

Beagles, 51.

Bedlington Terriers, 116.

Black-and-Tan Terriers, 106.

Black Mange, 178.

Blenheim Spaniels, 139.

Blinđness, 185.

Bloodhounds, 49.

Boar Hounds, 37.

Bones, broken, 191.

Borzois, 43.

Bouledoge Français, 146.

Bowels, Inflammation of, 170.

Bronchitis, 160.

Bull Dogs and Toy Bull Dogs,121.

Bull Terriers, 104.

Burns, 189.

Canker of the Ear, 185.

Cataract, 185.

Catarrh, 161.

Chesapeake Bay Dog, 143.
Chest Founder, 166.

Chorea, 163.

Chow-Chow, 102.

Clumber spaniel, 77.

Clydesdale Terriers, 119.

Cocker Spaniel, 82.

Collies, 85.

- Smooth, 91 .

Conformation, 32.

Coryza, see Catarrh.

Dachshund, 100.

Dalmatians or Carriage Dogs,

94.

Dandie Dinmont Terriers, 115.

Deerhounds, 41.

Dhole Dog, 149.

Diarrhœa, 171.

Dingo, 145.

Diseases and Accidents, 150.

Distemper, 150.

- Infection in, 155.

Dog, conformation of, 32 .

Dog-Breeding for Pleasure and Profit, 25.

Dog shows, and Preparation for Exhibition, 21. 


\section{INDEX}

Dropsy of the Flap of the Ear, Greyhounds, 55. 188.

Dysentery, 172.

Dyspepsia, 169.

Ear, Canker of, 185. 188.

- Eczema of, 183.

Eczema, Acute and Chronic, 174.

Elk Hounds, 149.

English Springer, 84 .

English White Terrier, 112.

Enteritis, or Inflammation of the Bowels, 170.

Epilepsy or Fits, 161.

Esquimaux Dog, 145.

Exercise, 9.

Eyes, Inflammation of (Ophthalmia), 184.

Feeding, 13.

Feeding Sick Dogs, 18.

Fever, Typhus, 171.

Field Spaniels, 79.

Fleas, 180.

Forelgn Dogs, 143.

Fox Terriers, Smooth and Wire, 188.

Fractures, 191.

Gastritis, ox Inflammation of the Stomach, 170.

Great Danes, 37.

- Italian, 127.

Griffon Bruxellois, 134.

Grooming, 10.

Harriers, 53.

Hind $\operatorname{Lim} b, 31$.

Indigestion, 169.

Inflammation of Lungs, 159.

Irish Terriers, 117.

Irish Water Spaniel, 72.

Italian Greyhounds, 127.

Japanese Spaniels, 136.

Jaundice, 158.

Kennels, 15.

Kennel Lameness, 166.

King Charles Spaniels, 141.

Lameness, Kennel, 166.

Lhassa Terrier, 147.

Lice, 180.

Lungs, Inflammation of, 159.

Maltese Terrier, 135.

Manchester Terrier, 105.

Mange, Black (Follicular), 178.

— Parasitic, 177.

Mastiff, 39.

Medicine, Administering, 17.

Mexican Hairless Dog, 148.

Newfoundland, 35 . 
Old English Sheep Dog, or Smithfields, 91.

Otter Hounds, 47.

Paralysis, 164.

Parasitic Mange, 177.

Pekinese Spaniels, 137.

Persian Greyhound, 148.

Pleurisy, 159.

Pointers, 57.

Points of Various Breeds, 32.

Poisoning Dogs, 173.

Pomeranians, 128.

Poodles, 97.

Prince Charles Spaniels, 139.

Pugs, 131.

Puppies, Rearing of, 19.

Rabies, 167.

Rearing Puppies, 19.

Retrievers, 63.

- Curly coated, 64.

- Flat coated, 66.

- Labrador, 68.

Rheumatism, Acute and

Chronic, 166.

Ruby Toy Spaniels, 139.

Russian Wolf-Hound, 43.

St. Bernards, 33.

St. Vitus's Dance, see Chorea.

Samoyede Dog, 144.

Scalds, 189.

Schipperkes, 126.

Scottish Terriers, 110.
Selecting Dogs for Companions, 22.

Setters, 60.

Skye Terriers, 119.

Smithfields, 9.

Soap, 13.

spaniels, Blenheim, 139.

- Clumber, 77.

- Cocker, 82.

__ English Springer, 84.

— Field, 79.

—_ Irish Water, 72.

_- Japanese, 136.

_ King Charles, 139.

- Prince Charles, 139.

—- Ruby Toy, 139.

- Sussex, 81.

- Thibet, 149.

- Toy, 137.

- Tri-coloured, 139.

Water, 75.

Welsh Springer, 83.

Spaniel Club Rules, 73.

stomach, Inflammation of, 170.

Terriers, Airedale, 113.

- Australian, 149.

Bedlington, 116.

Black and Tan, 106.

- Bull, 104.

Clydesdale, 119.

Dandie Dinmont, 115.

English White, 112. 


\section{INDEX}

Terriers, Fox, 108.

- Irish, 117.

- Lhassa, 147.

- Scottish (West Highland), 110.

- Skye, 119.

— Welsh, 114.

- Yorkshire, 124.

Thibet Mastiffs, 149.

Thibet Spaniels, 149.

Ticks, 180.

Toy spaniels, 137.

Typhus Fever, 170.

Washing, 12.

Water Spaniels, 75.

Welsh Springers, 83.

Welsh Terriers, 114.

West Highland White Terriers, 110.

Whippets, 96.

Worms, 1 s1.

Wounds, 189.

Yorkshire Terriers, 124. 
LRBAF" 



LIBRARY OF CONGRESS |||||||||||||||||||||||||||| 000327429190 